\title{
Circles of Trust: A Comparison of the Size and Composition of Trust Circles in Canada and in China
}

\author{
Jianghe Niu \\ A thesis submitted to the \\ Faculty of Graduate Studies and Research \\ In partial fulfillment of the requirements for the degree of \\ Doctor of Philosophy
}

\author{
Department of Psychology \\ Carleton University \\ Ottawa, Ontario
}

May 2006

(C) Jianghe Niu 2006 


$\begin{array}{ll}\begin{array}{l}\text { Library and } \\ \text { Archives Canada }\end{array} & \begin{array}{l}\text { Bibliothèque et } \\ \text { Archives Canada }\end{array} \\ \begin{array}{l}\text { Published Heritage } \\ \text { Branch }\end{array} & \begin{array}{l}\text { Direction du } \\ \text { Patrimoine de l'édition }\end{array} \\ \begin{array}{l}\text { 395 Wellington Street } \\ \text { Ottawa ON K1A 0N4 }\end{array} & \begin{array}{l}\text { 395, rue Wellington } \\ \text { Ottana ON K1A ON4 } \\ \text { Canada Oa }\end{array}\end{array}$

Your file Votre référence ISBN: 978-0-494-23297-2 Ourfile Notre référence ISBN: 978-0-494-23297-2

NOTICE:

The author has granted a nonexclusive license allowing Library and Archives Canada to reproduce, publish, archive, preserve, conserve, communicate to the public by telecommunication or on the Internet, loan, distribute and sell theses worldwide, for commercial or noncommercial purposes, in microform, paper, electronic and/or any other formats.

The author retains copyright ownership and moral rights in this thesis. Neither the thesis nor substantial extracts from it may be printed or otherwise reproduced without the author's permission.
AVIS:

L'auteur a accordé une licence non exclusive permettant à la Bibliothèque et Archives Canada de reproduire, publier, archiver, sauvegarder, conserver, transmettre au public par télécommunication ou par l'Internet, prêter, distribuer et vendre des thèses partout dans le monde, à des fins commerciales ou autres, sur support microforme, papier, électronique et/ou autres formats.

L'auteur conserve la propriété du droit d'auteur et des droits moraux qui protège cette thèse. $\mathrm{Ni}$ la thèse ni des extraits substantiels de celle-ci ne doivent être imprimés ou autrement reproduits sans son autorisation.
In compliance with the Canadian

Privacy Act some supporting forms may have been removed from this thesis.

While these forms may be included in the document page count, their removal does not represent any loss of content from the thesis.
Conformément à la loi canadienne sur la protection de la vie privée, quelques formulaires secondaires ont été enlevés de cette thèse.

Bien que ces formulaires aient inclus dans la pagination, il n'y aura aucun contenu manquant. 


\begin{abstract}
The purpose of this study was to explore the utility of the trust circle, an original approach which is characterized by trust circle size (how many people are trusted) and trust circle composition (who are trusted and what positions these trustees take). This conceptualization was tested in two domains: economic /financial risk settings, as well as in positive and negative personal/social information sharing at different variants of risk in each of these domains. Questionnaire data were collected in two different cultures (Canada and China), with the expectation that the size and composition of the trust circle might differ due to difference in culture and general social structures, frequently referred to in the literature on individualism and collectivism. The exploration was conducted by looking at the 2 trust loci of "I-trust-others" and "others-trust-me" in the same sample. One hundred Chinese residents of the People's Republic of China and 102 Canadians, half of them university students and the other half members of the general population participated in the study. A set of self-report questionnaires created in a pilot study was given to participants to establish domains of trust, and a range of trustees.

It was found that the concepts of domain-based trust, trust circle and locus of trust are important and effective concepts to use in better understanding trust in new ways and determining how people distribute their trust differentially. Trust is variable, trust circle size and trust circle composition tend to differ, if any one of the following factors differs: the domain concerned (economic/financial or personal/social); within the domains of economic/financial risk-taking, the risk level in lending or borrowing; within the domain of personal/social information sharing, whether the shared information is negative or positive, the variants of the positive and negative valence; the locus of "I-trust-others" or
\end{abstract}


"others-trust-me" and whether the involved culture differs. For example, when facing a higher economic/financial risk domain, Chinese trust more others than Canadians. However, compared with Canadians, Chinese are more likely to trust fewer others, not only in a negative personal information sharing domain but also in a positive domain. In all circumstances, it would appear that Canadians place higher trust in spouse than do Chinese. Results also give support to the utility of a framework for studying trust and reciprocal trust, since trust circle size and trust circle composition when placing trust in others tend to be different from the trust circle size and trust circle composition in the locus of others-trust-me, for example, people tend to trust a parent/guardian, grandparent, boss /supervisor /teacher and psychologist, all of whom are members of older generations and superiors, more than those individuals trust them. In contrast to this, people believe that they trust a spouse, child /grandchild, friend, classmate/co-worker/colleague, and acquaintance /neighbor, who are members of similar or younger generations and of equal or inferior status, less than those individuals trust them. Some results of the study were inconsistent with the concepts of individualism /collectivism and this may suggest that the complexity of a culture (such as China) may not be explained completely only by the individualism/collectivism paradigm. 


\section{Acknowledgements}

First, I would like to acknowledge my supervisor, Dr. Margaret Foddy, for her trust in letting me explore a new area/field and, with her supervision and guidance, I have learned a great deal about the importance of project pursuit, completion and writing.

I would also like to acknowledge each of my committee members: Dr. John Berry, external examiner, Queen's University, Dr. Zhiqui Lin, internal examiner, Department of sociology and Anthnopology, Dr. Warren Thormgate, Dr. Michael Wohl, who provided a helpful critical eye and valuable comments to improve the thesis.

I would like to thank Statistics Instructors Dr. Craig Leth-Steensen and Bruce Hutcheon in our department, who discussed related statistical issues with me on their own time, as well as Graduate Supervisor Dr. John Zelenski, and Graduate Administrative Staff Etelle Bourassa, Natalie Pressburger whose kind work supported the research.

I would also like to thank all participants, all friends, classmates participating in the study, commenting on the paper, and offering ideas, and so on.

Thanks to dear Nicole Chartrand, you have been going with me for the whole process, you helped me those "a/the", "affect/effect", ", /;" and marked "?" for "what does it mean" and so on. From spring to winter, from morning to night you always were there to provide your volunteer help again and again.

And finally, thank you to my parents and family for their undying love and constant support throughout the stages of my time at Carleton. You are right behind me, really concerned with my progress, your understanding and unconditional trust provided the fuel that has allowed me to continue on this journey, without you, I would never have completed my Ph.D. 


\section{Table of Contents}

Abstract.........................................................

Table of Contents.................................................... iv

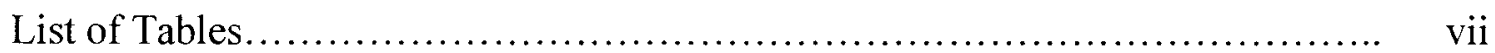

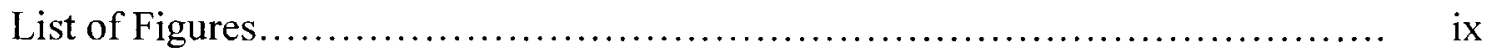

List of Appendices................................................... xi

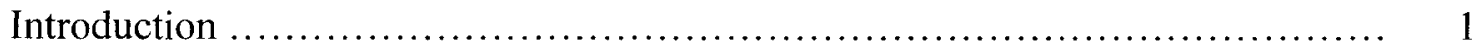

Overview....................................................... 1

Trust............................................................. 12

What Has Been Done in Related Trust Research....................... 16

Trust Circle........................................................ 23

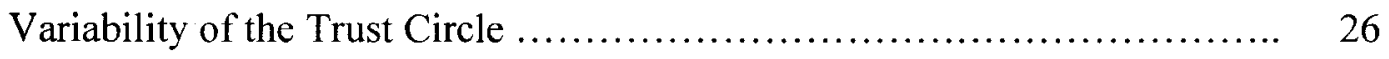

Trust Circles in Different Cultures ................................. 31

Purpose and Research Design ........................................... 37

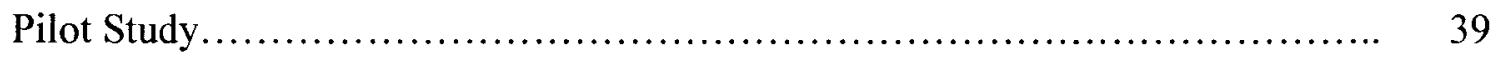

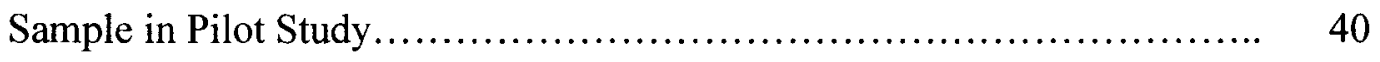

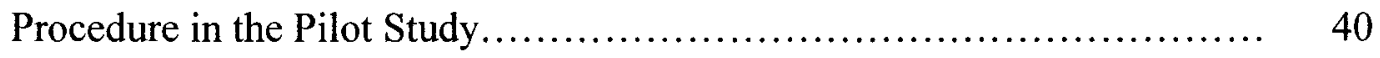

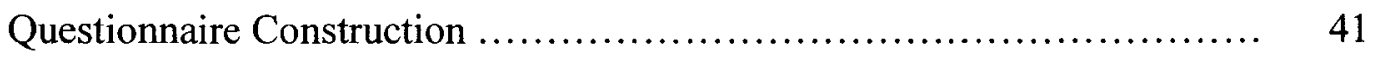

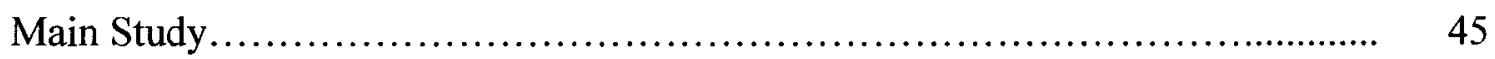

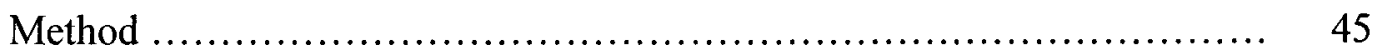

Participants............................................... 45

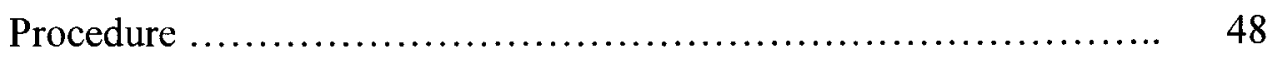


Trust Circle in the Economic Risk Domain.

Results and Discussion.......................................... 51

Trust Circle Size in the Economic Risk Domain.................. 51

Trust Circle Composition in the Economic Risk Domain............. 53

Cultural Effects on Trust Circle Size in the Economic Risk Domain... 57

Cultural Effects on Trust Circle Composition in the Economic

Risk Domain............................................ 61

Age, Education Level, Family Income, Gender, Participant's Status and the Trust Circle in the Economic Risk Domain............ 67

Conclusion about the Trust Circle in the Economic Risk Domain............ 69

Trust Circle in the Personal Information Sharing Domain...................... 70

Results and Discussion.......................................... 71

Trust Circle Size in the Personal Information Sharing Domain.......... 73

Trust Circle Composition in the Personal Information Sharing

Domain 76

Cultural Effects on Trust Circle Size in the Personal Information

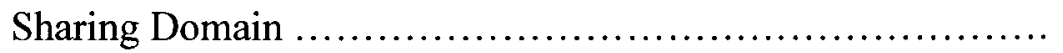

Cultural Effects on Trust Circle Composition in the Personal

Information Sharing Domain

Age, Education Level, Family Income, Gender, Participants' Status

and the Trust Circle in the Personal Information Sharing

Domain

Conclusion about the Trust Circle in the Personal Information Sharing 
Domain.

Trust Circle in Domain-based Trust..................................... 98

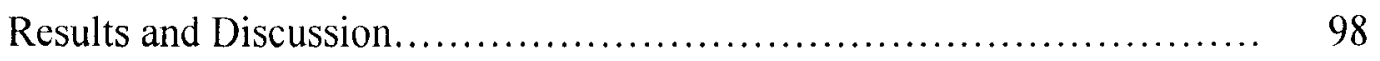

Trust Circle Size in the Economic Risk and Personal

Information Sharing Domains ......................... 98

Trust Circle Composition in the Economic Risk and Personal

Information Sharing Domains $\ldots \ldots \ldots \ldots \ldots \ldots \ldots \ldots \ldots \ldots . \quad 100$

Cultural Effects on the Trust Circle Size in Economic Risk

and Personal Information Sharing Domains................ 104

Cultural Effects on the Trust Circle Composition in the Economic

Risk and Personal Information Sharing Domains........... 107

Conclusion about the Trust Circle in Domain-based Trust .............. 111

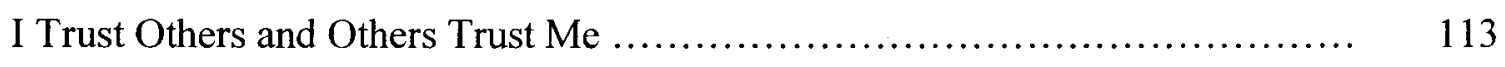

Results and Discussion......................................... 117

Size of the Trust Circle: Comparing "I-trust-others" and "Others-

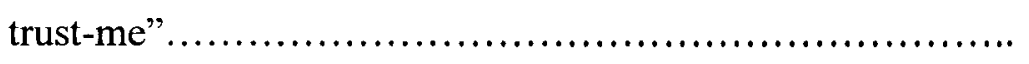

Composition of the Trust Circle: Comparing "I-trust-others" and

"Others-trust-me" ................................. 120

Conclusions about the Trust Circle in the Two Trust Loci.............. 124

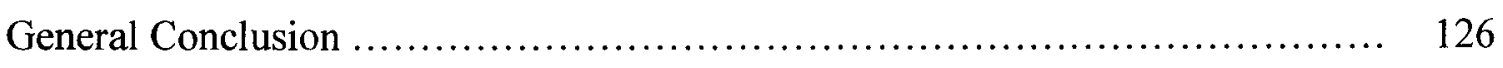

Limitations and Future Research........................................ 130

References......................................................... 134

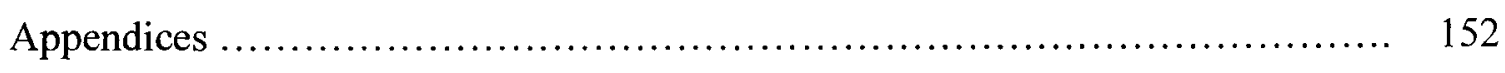




\section{List of Tables}

Table 1: Ranks and Proportions of Trustees at the 4 Economic Risk

Levels

Table 2: Trustee Chosen Proportions and Standard Deviations of Canadians and

Chinese in the Economic/financial Risk Domain

Table 3: Discriminations of Trustee between Canadians and Chinese in the Economic /financial Risk Domain

Table 4: Ranks and Proportions of Chosen Trustees at the 4 Different Positive Information Variants

Table 5: Ranks and Chosen Proportions of Trustees at the 4 Different Negative Information Variants

Table 6: Pair Trustee Proportion Differences of the 4 Trustee Categories within Positive and Negative as well as between Positive and Negative Personal Information Sharing Domains

Table 7: Trustee Chosen Discriminants between Canadians and Chinese in the Positive and Negative Personal Information-Sharing Domains 90

Table 8: Proportions, Standard Deviations and Differences of the 4 Trustee

Categories between Canadians and Chinese in the Positive and Negative Personal Information Sharing Domains.

Table 9: The First 4 Top Trustee Chosen Proportions of Canadians and Chinese in the Economic Risk, Positive and Negative Information Sharing Domains

Table 10: Trustee Ranks and Proportions in the Loci of "I-trust-others" and 
"others-trust-me" in the Economic Risk, Positive and Negative Personal

Information Sharing Domains......................................

120 


\section{List of Figures}

Figure 1: Mean Trustee Numbers at the 4 Economic Risk Levels............... 51

Figure 2: Mean Trustee Numbers of Canadians and Chinese at the 4 Economic

Risk Levels .................................................... 58

Figure 3: Fourteen Trustee Proportions of Canadians and Chinese at the

Economic/financial Risk Domain ................................... 62

Figure 4: Mean Trustee Numbers of People with Lower and Higher Levels of

Education in the 4 Economic/financial Risk Levels

Figure 5: Fourteen Trustee Chosen Proportions of People with Lower and Higher

Education Levels in the Economic/financial Risk Domain

Figure 6: Mean Trustee Numbers in the 4 Positive (left) and Negative (right)

Variants of Personal Information Sharing Domains.

Figure 7: Proportions of 14 Trustees in the Positive and Negative

Personal Information Sharing Domains

Figure 8: Comparing Proportions of the 4 Trustee Categories Chosen in the

Positive and Negative Personal Information Sharing Domains.

Figure 9: Mean Trustee Chosen Numbers of Canadians and Chinese in the Positive and Negative Personal Information-Sharing Domains

Figure 10: Proportions of Trustees Chosen by Canadians and Chinese in the Positive Personal Information-Sharing Domain.

Figure 11: Proportions of Trustees Chosen by Canadians and Chinese in the Negative Personal Information-Sharing Domain......................

Figure 12: Four Trustee Categories Chosen Proportions of Canadians and Chinese 
in the Positive (left) and Negative (right) Personal InformationSharing Domains

Figure 13: Mean Trustee Chosen Numbers at the 4 levels of Economic Risk Domain (left), Positive (middle) and Negative (right) Personal Information-Sharing Domains

Figure 14: Fourteen Trustee Chosen Proportions in the Economic Risk, Positive and Negative Personal Information-Sharing Domains

Figure 15: Chosen Proportion of the 4 Trustee Categories in the Economic Risk, Positive and Negative Personal Information Sharing Domains 102

Figure16: Mean Trustee Numbers Listed by Canadians and Chinese in the Economic, Positive and Negative Personal Information-Sharing Domains Trustee 105

Figure 17: Four Categories Chosen Proportions of Canadians and Chinese in the Economic Risk (left), Positive (middle), and Negative (right) Personal Information Sharing Domains

Figure 18: Mean Trustee Numbers for the Loci of "I-trust-others" and "otherstrust-me" in the Economic Risk, Positive and Negative Personal Information Sharing Domains 


\section{List of Appendices}

Appendix A: When it Comes to Economic Matters, Whom Would You Trust? ..... 152

Appendix B: When it Comes to Positive Matters, Whom Would You Trust? ...... 155

Appendix C: When it Comes to Negative Matters, Whom Would You Trust? ..... 158

Appendix D: When it Comes to Economic Matters, Who Would Trust You? ...... 161

Appendix E: When it Comes to Positive Matters, Who Would Trust You? ........ 164

Appendix F: When it Comes to Negative Matters, Who Would Trust You? ....... 167

Appendix G (Chinese version): When it Comes to Economic Matters, Whom

Would You Trust? ............................................... 170

Appendix H (Chinese version): When it Comes to Positive Matters, Whom

Would You Trust? ............................................. 173

Appendix I (Chinese version): When it Comes to Negative Matters, Whom

Would You Trust? ........................................... 176

Appendix J (Chinese version): When it Comes to Economic Matters, Who

Would Trust You?

Appendix K (Chinese version): When it Comes to Positive Matters, Who Would

Trust You? ...................................................... 182

Appendix L (Chinese version): When it Comes to Negative Matters, Who Would

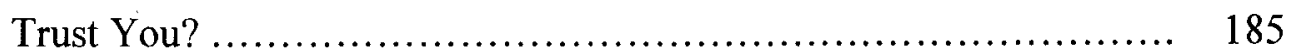




\section{INTRODUCTION}

\section{Overview}

Without a doubt, trust plays a very important as well as irreplaceable role in our lives and some form of trust is necessary for the continued operation of any society and culture. Trust becomes a more urgent and central concern in today's contingent uncertain global conditions since our world has become more interdependent. The ongoing process of global interdependency will increase the demand for trust in a wider range of others as an essential condition for cooperation. Because social life is pervaded with new and expanding threats and hazards of our own making, coping with raised vulnerability in the "risk society" requires an enlarged pool of trust (Sztompka, 1999a). Various relationships and different behaviors are also constructed on the basis of trust. Trust leads to many positive outcomes, such as greater willingness to cooperate (Yamagishi, Makimura, Foddy, Massuda, Kiyonari \& Platow, 2005), less risk of failure or betrayal, maintenance of longer relationships (Tilly, 2005), improvement in organizational effectiveness (Whitener, 2006) and better understanding of different cultures ((Luo, 2002; Zhang \& Bond, 1993) and so on.

Trust is particularly relevant in conditions of uncertainty with respect to unknowable actions of others; in other words, trust becomes an indispensable strategy to deal with uncertainty in our social environment. Without trust we would be paralyzed and unable to act in risky uncertain situations in which our outcomes depend in part on others (Bohnet \& Zeckhauser, 2004; Fisler, 2005). One could also state that trust is the first step for building various relationships and, based on trust of each other, further interaction between people is possible (Thye, Yoon \& Lawler, 2002; Tyler, 2003a). 
Sometimes, there is more than one relationship between an individual and other individuals (e.g. student vs. teacher, patient vs. counselor, child vs. parent), between this group and that group (e.g. Tom's family vs. a hockey group, Chinese vs. Canadian), between the individual and several groups (e.g. a woman in a dance group, an employee in a trade union, a Chinese person in an immigrant group) at the same time (Brewer \& Roccas, 2001). If people do not trust each other, as individuals or as members of a generalized group, people will be reluctant to enter into new relationships, a man cannot be the student of a professor, a woman cannot be the wife of a man, and people do not join groups.

Many scholars consider that trust is the belief that another's future behavior will be favorable, or at least not detrimental, to one's interests (Morrison \& Robinson). Trust is an expectation of goodwill and benign intent. Trust is also based on the supposition of the partner's personal traits and intentions (Brann \& Foddy, 1987; Foddy, 2003; Yamagishi \& Yamagishi, 1994). But the anticipatory belief is not sufficient to speak of there being trust, since trust is more than just contemplative consideration of future possibilities; we must also face the future actively by committing ourselves to action with at least partly uncertain and uncontrollable consequence (Sztompka, 1999b). "Trust is the correct expectation about the actions of other people that have bearing on one's own choice of action when that action must be chosen before one can monitor the actions of those others" (Dasgupta 1988, p.51).

Here trust involves commitment through action; that is, trust is related not only to another's action but also to his/her own action. For instance, I trust this politician to rule wisely, hence I vote for him, I trust Diana to be a good wife in the future, hence I marry 
her, I trust David to be able to keep my secret, so I tell him my secret. We only give house keys to those we believe are honest, and we only lend money to those we believe will return it. Trust obviously appears in the context of human actions; in the present study, trust is explored by people's potential actions.

All people must at some time trust some others, some people trust more often, while some people are more hesitant. People do not, will not and cannot trust everyone, and people also do not and cannot trust only themselves. The number of trustees could be described as trust circle size and the trust circle size is important to understand and describe trust.

In addition, it is necessary to consider who is included in the trust, it reflects a certain relationship to the focal person; further, even if you and I all trust the same number of trustees in a given set of circumstances, your trustees may be family members, my trustees may be friends, teachers etc. and even the same trustees may be trusted differently for hierarchy: one person may place parents, spouse, and children as most trusted, but parents more than spouse, more than children while another person may trust spouse first, parents next, and children third. One can see that there will be a hierarchy of trust in those in the trust. The relationship and the hierarchy (order of trusting) will be described as the trust circle composition, which is also important to help us better explain and understand trust.

Then a new concept of the "trust circle" is presented here in a way that has not been previously developed in the social psychology literature. The trust circle includes two aspects: 
1. The size of the trust circle, which represents the number of trustees being trusted; a bigger trust circle size means more others are trusted than in a smaller trust circle.

2. The composition of the trust circle, which refers to the types of people included within the trust circle, that is a kind of relationship to the focal person reflecting the nature of individuals who are trusted and the hierarchy of trust, that is the order of trusting, being trusted first, secondly, thirdly etc. reflecting how those trustees are trusted.

Both trust circle size and trust circle composition make up the trust circle and then identify the number of people in the trust circle, as well as the relationship to the focal person and their hierarchy of trust. The concept, without doubt, can improve the present description and explanation of trust. Because the size and composition describe the two very important factors of trust process, which had not been discussed before, and the size and composition of the trust circle are variable factors, they can therefore reveal variable natures of trust across differing content of the material being entrusted, across different levels of trust and in different cultures.

There are several studies which touch upon group size; for example, Sato (1988) noted that the effect of trust is hypothesized to diminish as the group size increases. In an experiment conducted by Houlette, Gaertner, Johnson, Banker, Riek \& Dovidio (2004), participants took part in a program designed to widen their circles of inclusion to include more other people. Some studies also touch upon the types of trustees and the relationship to the focal person: for instance, Buchan and Croson (2004) examined trust boundaries using different people in their experiment, such as parents, siblings, cousins, students you know well etc. 
However, the trust circle in this study is different from that appearing in previous trust studies. First of all, the concept of the trust circle always includes 2 parts, trust circle size and trust circle composition, rather than only one; the size of the trust circle reflects trustee numbers, not just group numbers; further, the composition of the trust circle reflects not only who will be in the circle but also what hierarchy those trustees will indicate.

Do people always trust the same people, whatever the circumstances? Hardin (2001) notes that I might ordinarily trust you to keep even the most damaging gossip confidential, but not with the price of today's lunch, while I trust another with the price of lunch but not with any gossip. I might trust very few people with almost anything and many others with almost nothing. From Hardin's example here, one can glimpse the meanings of trust circle size and composition in different situations. Because Hardin realizes that a changeable relationship between trust and the related background exists, trust depends on context, however, he does not offer a scheme to characterize these differences.

I believe that trust may not be a static thing, staying the same in various contexts or circumstances; the trust circle may not always be the same against different backgrounds. It appears that the trust circle is the outcome of various independent situational variables, given that many relevant factors, such as culture, socioeconomic and social structural conditions change (Kashima, 2002; Kashima \& Foddy 2002). When such factors differ, it is likely to produce differences in forms of trust, as the background varies, the trust circle varies correspondingly. 
Several researchers have paid attention to the context of trust (Child \& Mollering, 2003; Hardin, 2001; Rousseau, et al., 1998). The important question is not only "how much do I trust?" but also "in what areas and in what ways do I trust?" (Rousseau, Sitkin, Bunt, \& Camerer, 1998, p. 395). A related question, "who would trust me", will be discussed later.

I will make the case that we should choose those examples which best represent categories or types of situations since we cannot study all domains. There is no existing typology for "domains of trust", and the basis of choice must be, at this initial stage, somewhat arbitrary. For this study I will concentrate on two domains which are regarded as "different" domains. One part belongs to the economic, financial life, which is the outside material world, while the other belongs to the personal, social, psychological life, which represents a less-material world. Both of these involve relationships between people and, of course, both of these involve issues of trust. By careful selection of settings which exemplify these "different types", I choose two specific examples to represent the economic/social and personal/social life. To keep succinct, I will only use economic to denote economic/financial, and personal in place of personal/social for the following parts.

The trust circle will also be examined in different cultural backgrounds because all human behavior is culturally patterned. Explanations of human diversity are sought in the cultural context which people have experienced. As a cultural and historical phenomenon, the concept of trust, as well as corresponding trust behavior, may be substantially different in various social and cultural systems (Earle \& Cvetkorich, 1995; Fukuyama, 1995). 
Culture is a shared meaning system that may unite the people within it, and it often excludes other people (Yoshino, 2002). Berry, Poortinga, Segall and Dasen (2002a) offer a concise definition: "culture is the man-made part of the human environment". Indeed, different environments represent different cultures, this may partly explain why social behavior in one culture differs from that in another. It is also believed that cultures are shapers of human behavior, that is humans are producers of culture and, at the same time, are influenced by it (Kashima, 2002; Segall, 1999; Triandis \& Trafimow, 2001). It should be noted at this point that the term "culture" has also been used to refer to subcultures, such as "ethnic culture", "the culture of the deaf", and so on. While the term "culture" is used in a similar way, for purposes of this thesis, I will deal only with culture as it is aligned with broad national groups.

Many kinds of behavior occur in almost all cultures, all human behavior is cultural to some extent. For example, greetings take place in all cultures, and people all over the world trust some others, showing that such behaviors are pretty universal. On the other hand, the particular social behaviors are obviously linked to the particular cultural context in which they develop; for instance, actual greeting procedures vary widely, from hand shaking in China to embracing in the West to bowing in Japan, and they are shaped, influenced and developed by different cultures (Berry, Poortinga, Segall \& Dasen, 1992). Similarly, national differences in trusting behavior exist (Buchan, Croson \& Dawes 2002; Wang \& Yamagishi, 2005). As long as the cultures differ, trust behavior may not be the same in each. The central concept of "trust" may be universal (an expectation that others will treat you benevolently, or not harm you), but the grounds on which trust is extended may vary. 
In some societies, people are more ready to grant trust, while in other societies people may be more hesitant to trust others, or restrict trustors to a smaller trust circle. Looking at trust as a cultural phenomenon is the domain of the cultural approach, since culture becomes a powerful factor influencing decisions to trust.

As globalization becomes more common, more and more people migrate from one country to another, from one place to another, and societies become more intertwined (Buchan, et al., 2002). As well, people all over the world interact with each other much more often and in more meaningful ways than before and the world we live in has undergone significant changes over time as traditional societies/less developed societies change into modern/more developed societies (Kashima \& Foddy, 2002), it is thus important to understand how others' trust differs from our own. It is not within the scope of this thesis to trace historical changes in the basis and forms of trust, but rather to use cross-cultural comparisons to examine whether identifiable differences in different cultures have an impact on the size and composition of the trust circle, since culture is a set of knowable regularities, characterises human groups (Hoorens \& Poortinga, 2000; Munroe and Munroe, 1994).

Human behaviors are seen as products of cultures, as well as shapers of culture. Understanding how humans modify culture and how various cultures modify humans is important knowledge in every social science field. Each nation or race has its own culture, social values and ways of thinking, and these underlie each nation's system of economics, politics and social life (Markus \& Kitayama, 2003; Segall, 1979). Cultures are likely to differ in the way individuals are shaped to extend trust, and to act in a trustworthy fashion. 
In theory, choosing any culture for the cross-cultural trust circle study would be fine, however it is not possible to choose every culture to study. Thus, how to choose a sample is the first and important step of the study. Berry et al (2002b) pointed out that there are two approaches to choosing cultures for comparison. One approach involves selecting some cultures which can be considered representatives of all the cultures in the world; the other approach involves choosing only a few cultures which clearly differ on some variables that provide a contrast. Because it is hard to know which cultures can represent all cultures in the world, or to study all of them, in this study, the approach was to choose clearly differing cultures.

Because this study is a study of exploration and possible relevance between some components of culture and trust are not well-known yet, the best way to choose a cultural sample is to consider those cultures which express as many differences from each other as possible. Thus Canada and China were selected.

Berry et al. (2002a) distinguished six general cultural areas: sub-Saharan Africa, Asia, Australasia, circum-Mediterranean, North America, and South America. Canada and China belong to Asia and North America respectively. In many dimensions of interest to psychologists (e.g., literacy, socialization practices, collectivism), the range of variation within these cultural areas is about as large as it can be.

When considering cultural areas based on geographical distribution, East and West, China and Canada are representative of two major and distinctive cultures of the East and the West (Triandis, Brislin, \& Hui, 1988; Yuki, 2003).

Race, reflecting skin color, place of recent ancestral origin, parental language, religious identity, or whatever determinant matters socially, has led to some systematic 
genetic divergences and has also been used as an explanation for visible physical and behavioral differences. Among them, skin color is easily the most distinguishable characteristic on which human groups differ genetically (Segall, 1999). Canadians and Chinese, without a doubt, are two difference races and their skin color, generally, is different.

Speaking different languages may be a factor in promoting human behavioral diversity (Berry et al., 2002c). The languages most Canadians and Chinese speak, write and use are totally different.

The choice is also based on the ranks of the aggregate Foreign Policy (FP) globalization index ((Buchan, 2005); the FP index includes rankings of 62 countries in four categories: economic integration, personal contact, technological connectivity, and political engagement. Canada's FP index is 6 and China's is 57 (the higher the ranking, such as 6 , the more globalized is that country, and the lower the ranking, such as 57 , the less globalized is that country). One can see a significant difference between the two cultures based on the FP index.

Thus, based on the reasons mentioned above, Canada and China have been chosen as cultural samples for this study since they appear to differ from each other quite dramatically and there are not studies comparing the two countries on the issue of trust thus far.

This dissertation will examine whether or not the size and composition of the trust circle differs in different domains (e.g. the economic risk domain and the personal information sharing domain), in situations characterized by different content (e.g. positive and negative shared personal information) as well as in different levels of trust 
required (due to the risk involved should the trust be violated), for example in the financial domain, this risk is operationalized as amount of lending money involved. Furthermore, I will also seek to find out whether the size and the composition of the trust circle change in different ethnic-cultural groups.

"How I trust others" is the natural way for both researchers and participants to think of trust; however it is just one side while "how others trust me" is another side which is also an interesting question, worthy of exploration. I believe that it is only by looking at both trustworthiness directions (from me to others, from others to me) that we can see the whole trust picture. Unfortunately, there is little or no directly related research on this topic, so the study was designed to examine the trust circle from both perspectives, or loci to provide additional insight into this reciprocal trust relationship.

The above is a short general overview. The next part will begin with a literature review of trust research. Then the concept of the trust circle will be defined and discussed in detail and, while pointing out the limitations of trust research and the lack of research concerning the "trust circle", the aims of this dissertation will be presented. After that, cross-cultural studies will be reviewed for the insight they provide about possible cultural differences of trust and related phenomena that might influence trust. After this, a pilot study, which provided the questionnaire for the dissertation, will be discussed briefly, followed by a description of the method used in the main study. The following results and discussion of the main study will be organized into the 4 sections: 1 . The trust circle in economic domains; 2 . The trust circle in personal information sharing domains; 3 . The trust circle in domain-based trust (comparing economic risk and personal information 
sharing domains); 4. Similarities and differences between the loci of "I-trust-others" and "others-trust-me".

\section{Trust}

To date, it seems that there is no unified and universal definition of trust recognized by every expert. For example, Foddy (2003) believes that trust is someone's expectation that another or others will perform an action which either is beneficial or is not detrimental to us. Tyler (2001) points out that one of the important aspects of trust is the prediction of the future behavior of others; that is, people are concerned about what others will do, what their future behavior will be. Similarly, trust is defined as an expectation that partners and/or potential partners will have goodwill when they deal with us (Yamagishi \& Yamagishi, 1994).

Hardin (2001) defines trust as a relation of three-parts: person A trusts person B to do X; here, the concept of trust is a dyadic one, concentrating on specific actions, in which all three parts are necessary for a whole trust process. Kramer (1996) believes that trust is related to cognition and influenced by hierarchical social relationships. On the other hand, Cook and Cooper (2003) consider trust at the micro level of society and have investigated the role of trust as a facilitator of cooperation and informal social exchange. The diversity of definitions of trust suggests that the trust concept is not simple and easy to define but rather is complex and multi-layered.

Even though we have no universally accepted definition of trust, there are still some important and basic factors mentioned by most researchers and scholars. That is trust is important in a number of ways: a core aspect of most definitions of trust is the "intention to accept vulnerability based upon positive expectations of the intentions of the 
behavior of another" (Rousseau et al, 1998, p.395). This definition will be adopted in this thesis.

Mayer, Davis and Schoolman (1995) name both parties of trust in their definition of trust with an expectation perspective. They indicate that trust is defined as the willingness of a truster to be vulnerable to the actions of a trustee based on the expectation that the trustee will perform a particular action important to the truster (Mayer, et cal., 1995). It seems that "trust is a psychological state comprising the intention to accept vulnerability based upon positive expectations of the intentions or behavior of another" (Rousseau, et al, 1998, p.395).

Once again, predicting the behavior of others is one of the most important aspects of trust (Tyler, 2001). Trust obviously appears in the context of human actions, as a whole, it depends on the future contingent actions of others, and follows one's own action/behavior as a consequence of trust. For example, people believe that the politician will fulfill his promises to them, hence they vote for him; the expectation of another's benevolent action and their own benevolent actions together comprise trust.

Most current views reflect the idea that trust is important in risky situations. There is agreement by most researchers on the conditions that must exist for trust to arise. Risk is one condition considered essential in psychological, sociological, and economic conceptualizations of trust (Rotter, 1967; Williamson, 1993; Yamagishi \& Yamagishi, 1994). The dependent connection between trust and risk taking arises from a reciprocal relationship: risk creates a necessity of trust, and trust decreases the perceived risk. Trust would not be needed if actions could be undertaken with complete certainty and no risk (Lewis \& Weigert, 1985). 
Yamagishi, Cook and Watabe (1998) use the term "social uncertainty" to refer to the risk of being exploited in social interactions. That is, social uncertainty usually exists when others may have an incentive and an opportunity to act in a way that imposes costs or harm on the person and when the person does not have enough information to predict whether others will behave in a positive way (Yamagishi et al., 1998).

The other condition of trust may be interdependence, where the interests of one party cannot be achieved without reliance upon another, since trust is about A to B with $X$, without any one of them, trust would not be happen.

Both risk and interdependence are required for trust to emerge (Sheppard \& Sherman, 1998). In other words, trust is the willingness to be vulnerable under conditions of risk and interdependence, generally speaking (Rousseau, et al., 1998).

Based on various viewpoints, researchers have classified trust into categories; several types of trust will be introduced here briefly in order to better understand trust. Relational trus. This type of trust derives from repeated interactions over time between truster and trustee (McAllister, 1995). This trust is viewed as an interpersonal or a relational phenomenon; thus, trust is initiated, built, enhanced, or destroyed through interpersonal transactions (Rousseau et al., 1998).

Group-based trust is a type of trust involving ingroup members, based on the fact that both truster and trustee belong to the same group. Yamagishi et al (2005) pointed out that people trust their own group members because they expect others to treat them well as a member of their group. This view is consistent with both "generalized exchange" (Buchan, Croson \& Dawes, 2002; Kiyonari, Tanida \& Yamagishi, 2000), and with social 
identity and self-categorization theory (Brown, 2000; Hogg, 2001; Turner \& Pratkanis, 1998).

Social Trust is linked to the role of groups in "providing people with information about their social identities" (Tyler, 2001, p. 302). Social trust is also related to social relationships and varies with those relationships (Tyler, 2003a). Social trust is an element of social capital. Sources of social capital include family, friends, neighbors, peers, and colleagues and social trust is also viewed as a property embedded in social relations in groups rather than in individual behavior (Welch, Rivera, Conway, Yonkoski, Lupton \& Giancola, 2005).

Identification-based Trust is more likely to depend on a person's identification (Maguire, Phillips \& Hardy, 2001). If people can construct themselves and others as possessing particular identities, then trust is possible. Also, when they are involved with identification-based trust, people show an increasing tolerance of exposure to risk and harm (Maguire et. al., 2001). Identification-based trust and group-based trust overlap in some situations, if identifying criteria are also the criteria to recognize a group; however, in many circumstances, they are different.

The types of trust mentioned above appear to share some common characteristics. For example, group-based trust is a kind of identification-based trust while social trust is also based on identity. The factor of the type of relationship is important not only in relational trust, but also in group-based trust, social trust and identification-based trust. Actually, social, group, identification-based and relationship trust are all linked to each other; these definitions have developed over time as researchers have worked to understand trust and it is not surprising there is some overlap. 


\section{What Has Been Done in Related Trust Researches}

Before I discuss the researches about the trust circle, variability of the trust circle, as well as trust circles in cross-cultural viewpoint, I would like to look back at what researchers and scholars have accomplished.

Group identity and trust have been investigated by many researchers (Dirks, 1999; Foddy, 2002; Tyler, 2003b \& Yamagishi et al., 2005; Yuki, Maddux, Brewer \& Takemura, 2005) to determine whether or not people's trust differs from ingroup to outgroup. Many of these studies employ laboratory settings with various forms of games in which people's outcomes depend in part on the behaviour of others. For example, Foddy (2002) asked whether or not there would be more entrustment if the other person was from the same group, even if the other was not personally known to the focal person. In order to address this question empirically, using existing categories of students (here, those majoring in similar or different fields), who had not, and would not meet each other, trust was assessed in terms of whether people showed preference to entrust their outcomes to an ingroup or outgroup "allocator", who, they were told, had allocated some or none of an endowment to people in the participants' group. The participants could choose the allocation of one of the two allocators without knowing until after the choice, from what the share of the allocators' funds had been, Foddy found strong preference for ingroup allocator, based on the belief that ingroup members would have allocated more to them. These results were interpreted to indicate that higher relative trust in strangers can be based in beliefs that category membership entails obligations to treat fellow group members benevolently. 
To some extent, all groups may discriminate social behavior between intra-group and inter-group. In other words, it may be universally true that people think that "we" are more trustworthy or honest than "they" (Brewer, 1999). This is likely to extend to the various bases of trust noted earlier, i.e., relational, group, category, etc.

The relationship between trust and cooperation, though not central to this thesis, had been studied extensively, and provides information about the dynamics of trust, particularly group-based trust (Brewer, 1999; Cook \& Hardin, 2001; Foddy, Yamagishi \& Platow, 2006; Yamagishi, 2003). The relationship between trust and cooperation was examined ingeniously by the following experiment. Two real participants were in a dyad: a sender and a receiver, each with some amount of money. All dyads were randomly split into two types: sender and receiver in the same group or in a different group (Foddy, 2002). In the experiment, the sender must trust the receiver to reciprocate while the receiver must want to give something to the sender-thus, they must cooperate (Berg, Dickhaut \& McCabe, 1995). Results of the experiment indicated that when the interactants in the dyads shared a common group membership, the levels of trust and cooperation were higher than when the dyads were not of the same group (Berg et al., 1995; Foddy, 2002). Similarly, Yamagishi (2003) pointed out that people were less cooperative when they played with an outgroup partner than with an ingroup partner. Among trust studies related to Americans, one study is most interesting. Brewer, Aday \& Gross (2005) examined Americans' generalized beliefs about how much the United States can trust other nations after the terrorist attacks of September 11, in 2001 and 2002. They found that the public's level of trust of Americans in other nations went from low in the immediate aftermath of September 11,2001 to even lower in the 
following summer. It is regretful that there are no data about what the level of international trust was prior to the terrorist attacks, and it is thus not possible to compare the trust before and after Sep. 11, 2001. In addition, the research did not identify what other nations specifically.

Do age, gender or education matter in issues of trust? The answer is yes. Here are some examples. Age similarity is positively correlated with trust. Younger executives tend to express a higher level of trust in their connections with each other in a Chinese sample (Farh, Tsui, Xin \& Cheng, 1998; Wang \& Yamagishi, 2005). Male subordinates with male supervisors expressed a lower level of trust in their supervisors than male subordinates with female supervisors (Farh, et al., 1998). Men would trust individuals based on whether or not they shared group memberships; on the other hand, women tended to trust those who shared direct or indirect relationship connections, which was found in a study sought to investigate differences in interdependence within the domain of trust conducted by Maddux and Brewer (2005).

Even though there are still untouched areas in trust research, trust has, up to now, been examined by scholars across a range of disciplines. It is worth mentioning at this point that the Russell Sage Foundation, as the principal American foundation devoted exclusively to research in the social sciences, has sponsored a large number of trust studies and has been publishing books containing the results, including Trust and Trustworthiness (Hardin, 2002), Trust in Society (Cook, 2001), Trust in the Law (Tyler \& Huo, 2002) and Cooperation Without Trust (Cook, Hardin \& Levi, 2005) etc.

Furthermore, trust research has been conducted not only in researchers' own cultures and nationalities, but also in cross-cultural settings. In essence, the core issues 
are whether "culture" and "behavior" are distinguishable entities, and whether the former is antecedent to, or even causes, the latter. Culture is what makes it possible for something to be a thing, and for a human being to be a person (Packer \& Tappan, 2001). It indicates that some cultures develop robust cultures of trust, whereas others are pervaded with distrust (Putnam, 1995; Rose, 1994). One should attempt to specify the wider cultural factors which are conducive to trust in the short and long term and see how their impact accumulates in establishing lasting traditions of generalized trust. As a kind of social behavior and a psychological tendency, trust is a constituent element of a given cultural system and cannot be separated from it, trust, as a result of culture, may therefore be different in various social and cultural systems (Earle \& Cvetkorich, 1995; Fukuyama, 1995; Yamagishi \& Yamagishi 1994).

Within the field of cross-national research, there are many more studies that have been conducted between Japanese and Americans. Some Japanese researchers are interested in exploring who (Japanese or Americans) will trust others more. For instance, Yamagishi (1988) pointed out, based on experiments and surveys, that Japanese participants had a lower level of trust in strangers and cooperated less in social dilemmas involving strangers compared to their American counterparts. American participants cooperated more often (contributing $56.2 \%$ of the resource money) than did Japanese participants (who contributed $44.4 \%$ of the resource money). Yamagishi \& Yamagishi found again in 1994 that Americans are more "trusting" than the Japanese as measured by comparison trusting of others in general between Japanese and Americans using a crossnational questionnaire survey (Yamagishi \& Yamagishi, 1994). Results of a later study conducted by Kiyonari and Yamagishi (1999) repeatedly showed a higher level of trust 
among Americans than among Japanese. This may seem counter-intuitive because the stereotype of Japanese is that they are very group-oriented; however, they may not necessarily be "group-oriented" when the group is a diffuse social category and not a group of individuals with ties of relationship and obligation.

Yamagishi \& Yamagishi (1994) considered this repeatedly confirmed result surprising to those who think that one of the significant characteristics of Japanese society is the stability of interpersonal and inter-organizational relations, especially in comparison to American society, and therefore it seems natural to conclude that stronger trust would have been nurtured in Japanese society. Yet this logical conclusion was inconsistent with the repeatedly confirmed results. In 2005, Yamagishi et al. also explored who, Australians or Japanese, would show stronger group-based trust, with finding no major difference found between Australian and Japanese participants. Another cross-national trust study compared trust among managers in Australia and China (Wang \& Clegg, 2002). They noted that Australian managers expressed more trust in subordinates' psychological maturity than did Chinese managers.

Many cross-cultural trust studies have recently used samples made up of Asians and Americans or Europeans. For example, Koreans and Danes were chosen by Kim, Helgesen \& Ahn (2002) as their representatives to study cross-cultural trust and they found that although both Danes and Koreans trusted close ingroup members, such as family members and friends, Koreans were much less likely to trust their colleagues and outgroup members. Japan and the United States were samples of East and West in a cross-cultural study of group-based trust conducted by Yuki, et.al., 2005), in the study, it was found that Americans tended to trust people primarily based on whether they shared 
category memberships; however, trust for Japanese was based on the likelihood of sharing direct or indirect interpersonal links.

There is some cross-national trust research related to China. A study concerned with supervisors' trust was conducted in China and the United States to explore the relationship between sales performance and supervisors' trust (Atuahene-Gima \& $\mathrm{Li}$, 2002). The study showed that supervisee trust enhanced sales performance for Chinese subjects when the supervisor possessed a higher level of achievement orientation style; for Americans, effects were negative or nonexistent for all examined variables excepting achievement orientation and supervisor accessibility. Another trust study concentrating on China and the United States considered the boundaries of trust (Buchan \& Croson, 2004); this will be discussed in detail below.

It is believed by many scholars that China, Japan and Korea are collectivist countries while the United States is an individualist country (Chirkov, Lynch \& Niwa, 2005; Huff, \& Kelley, 2003; Probst \& Lawler, 2006). Buchan and her colleague (2002) chose the samples for their study from China, Japan, Korea and the United States. The four countries participated in an experiment using the trust, or, sender/receiver paradigm based on Berg trust game; trust was measured by the amount of money sent by a sender to a receiver. They found that people in collectivist countries showed more trust than did the Americans. Also, in the three eastern countries, Chinese participants extended high levels of trust, Japanese participants extended low levels of trust, and Koreans trusted the least.

A study conducted to explore trust levels between racial/ethnic minorities and majorities in the United States provides some interesting results (Schnittker, 2004). From 
the results of the study, it appears that blacks and Hispanics are less trusting of their personal white physicians than are whites, and that education and income level both increase degree of trust placed in the physicians. That is, cultural or racial/ethnic diversity affects trust of others.

Recently, the term Guanxi has emerged from trust research related to China. The meaning of Guanxi in Chinese is closest to the meaning of "relationship" in English, but the two terms do not mean exactly the same thing. Guanxi is a type of interpersonal relationship, and built on a particular criterion, such as shared attributes, identity, or origin (Tsui \& Farh, 1997), or being relatives and close friends. Guanxi literally means relationship or connection becoming an enabler of transactions (Ramasamy, Goh \& Yeung, 2006), this/these relationships you can count on, you can rely on.

Some studies explore the links between guanxi and trust (Chen, Chen \& Xin, 2004; Chou, Cheng, Huang \& Cheng, 2006; Peng, 2001). The function of Guanxi in trust is not totally positive or negative, but rather is more complex. For instance, if Guanxi networks include non-job (unemployment), departmental and past team networks, it is found that the past team guanxi network of a team member displays a positive effect on the trust network, while non-job and departmental guanxi networks show negative effects on the trust network (Chou et al., 2006). To establish and enhance interpersonal trust, favoring a nephew of a hometown fellow would lower trust, but favoring a college schoolmate or a close friend would not (Chen et al., 2004). In order to investigate the strategies of producing interpersonal trust in Chinese society and the relations among such strategies, Peng (2001) conducted a study using a sample aged from 16-65 years asking participants to report the strategies they would use to enhance the trustworthiness 
of the focal person to scenarios. The results pointed out that a legal approach in conjunction with guanxi management can be used by Chinese in order to enhance interpersonal trust.

Among what had been done in related trust research, there is little known about the trust circle, let alone trust circle research in Canada and China. The aim of this study is to provide new knowledge by exploring the concept of the "trust circle" and its differences between cultures of the East and the Westj, the research is relevant within, as well as across, cultures. In addition, the investigation of loci of trust (I trust you, you trust me) will only be focused on relations in general, rather than discussed against a cultural background.

\section{Trust Circle}

A new concept, the trust circle, is defined and tested in the present study. The concept of "trust circle" includes two dimensions: 1. the size of the trust circle, which represents the number of trustees; 2 . the composition of the trust circle, which describes who will be in the circle and how they are trusted as reflected by the hierarchy of trust, or the order of trusting.

Human beings do not, will not and cannot trust everyone else, and they also do not and cannot trust only themselves; thus people must trust some others, if not all others and if not only themselves, how many others being trusted is therefore described in the trust circle with the trust circle size.

In addition, even if the circle size is the same, who is being trusted by you may be different from who is being trusted by me, for example, even if we both trust 3 others, you may trust 3 family members while I may trust 3 out-of-family members, as 
relationships to the focal person differ within the trust circle, furthermore, even when the same kinds of people (e.g. friend, teacher and psychologist) are trusted, there is an existing order of trusting issue; for example, you trust psychologist most, friend second, and teacher last, but it is vise verse for me. In order to describe these, we need trust circle composition to describe.

I believe that the new concept of "trust circle" can depicts trust in more detail than has previously been done, and it itself embodies the basic features of trust since trust is a relationship between people. Trust behavior involves 2 basic issues: 1 . how many people are trusted, which can be described by using trust circle size; 2 . the relationship between trustor and trustee, as well as the hierarchy of trusting (the order of trusting), which can be described by using trust composition. If we do not know the relationships of people in trust in addition to the number of people being trusted, how can we fully understand trust behaviors? Certainly, knowing the sizes of the trust circle and the composition of people in the trust circle will tell us more about trust than we know so far.

Again, even though there is plenty of trust research which covers political science (Boeckmann \& Tyler, 2003), business management (Jenkins \& Lawler, 1981), health systems (Cook et al., 2004) etc., as well as trust building (Cook et al., 2005) and trust maintaining (Kewicki \& Bunker, 1996), it appears that little is known about the size and composition of the group one trusts, in other words, the trust circle.

There are several studies which touch upon group size. For instance, the concept of "social distance", a measure of the closeness between players in a strategic interaction that has been acknowledged to have a profound influence on individual decisions (Akelof, 1997), and the circle of inclusion, based on a program which was designed to 
widen people's inclusion to include more people (Houlette et al., 2004) have inspired me to put forward my own opinions about the trust circle.

Whether or not trust varies in different sized groups has been examined in small and large groups (Draeger, 1969; Sato, 1988), but most of this research has been based on experimental studies. As an example of this research, Sato (1988) investigated whether the effect of trust on cooperation diminishes as group size increases. One hundred and seventeen Japanese undergraduates were assigned to a high- or low-trust condition and assigned to either 3- or 7-person groups. Participants were given money and asked to decide how much of it they wished to contribute, which would then generate benefits that would be equally shared by all members of their group. Results indicated that the positive effect of trust on cooperation exists only in small groups. In other words, the effects of trust diminished as the group size increased.

In order to help children widen their circles of inclusion to include people who are different from themselves based on race, sex, and weight, Houlette and his colleagues (2004) asked 830 first and second grade children to participate in a "Green Circle" program over four weeks. When children selected their preferred playmate, the researchers hoped that the children would draw their circle of social inclusion wider to take in others who had previously been excluded in other sessions and the results reached the purpose of the study, the program led children to be more inclusive in selecting their preferred playmate and the green circle became larger.

Fukuyama (1995) suggested that there exists a boundary of trust in different cultures or societies; people in relationships within that boundary are trusted and trustworthy, but people outside of that boundary are not. According to Fukuyama, this 
boundary in China is quite close-in, while in the US this boundary is much farther out. In order to test Fukuyama's idea, Buchan and Croson (2004) examined the boundaries of trust in the United States and China. In their study, Buchan and Croson created 7 types of trustees to represent different levels of social distance, they were: parent, sibling, cousin, fellow student you know well, student from a near-by university, stranger from your home town, stranger from another country.

Studies of the size of groups and the circle of social inclusion are related to the trust circle, and these researches provide useful information in the conceptualization of the trust circle. However, group size is not the same as trust circle size, and the circle of social inclusion is also different from the concept of the trust circle. The trust boundary seems close to the circle of trust but, again, it is not the same. Actually, a trust boundary is a rigid line, dividing those trusted from those not trusted. However the composition of the trust circle not only describes the relationship to the focal person within the trust circle, but also describes a hierarchy of trusting, who will be trusted most, who will be trusted second and so on. On the other hand, the trust circle is a dynamic circle which reflects the number of trustees, the relationship to the focal person, as well as the hierarchy of trusting. Both together form the overall trust circle.

\section{Variability of the Trust Circle}

The argument I wish to present is that trust is not a static thing, rather it varies across domains and degree of risk involved in placing trust in others. The question of how trust varies across time is beyond the scope of this thesis. Instead, the focus is on how trust varies in current, ongoing relationships. These may or may not be long or shortterm; the research reported here is concerned with what people report about existing 
relationships, which no doubt have a history, but the origin of these trusting relationships was not dealt with.

Hardin (2001) believes that trust depends on the context in which it exists. For example, a person who trusts you with gossip does not necessarily trust you on an economic issue. Several researchers have also paid attention to the context of trust (Child $\&$ Mollering, 2003; Hardin, 2001; Rousseau, et al., 1998). The important question is not "how much do I trust?" but "in what areas and in what ways do I trust?" (Rousseau, et al., 1998, p. 395) Indeed, "context is critical to understanding trust." Trust knowledge needs to address whether a particular context has influenced trust (Rousseau et al., 1998, p. 395).

However, it seems very little research has so far explored the relationship of trust and context. It is therefore the task of this dissertation to investigate the trust circle in a situational perspective, that is to explore how the people included in one's trust circle change with domain of trust, with valence of domain, with risk level, with whether one is a truster or a trustee, and whether these show any significant difference between cultures, an area in which one might expect differences in both the size and composition of the trust circle.

Like selecting cultural samples, as has been discussed before, it is necessary to choose a domain sample which is different and can thus provide two different backgrounds to explore whether the trust circle differs in different domains. Like the criteria for choosing culture, the main point for choosing is difference between the two domains. Generally speaking, some background in the material/financial domain differs as much as possible from some background in the personal information-sharing domain. 
While these two main domains are similar in that trust requires degrees of risk, they differ in the content of the "commodity" entrusted, and so for most purposes in this thesis.

In the two basic and different domains, we further need to narrow down specific situations that are suitable for the study, the situations can also be "ideal" and typical representatives of the economic risk and personal information-sharing domains.

Money plays an important role in people's material/financial life and it is also a basic part of people's economic life. How people handle money, whom they entrust their money to, and how people make up their trust circles are a typical domain in which to explore the trust circle in the economic context.

Most often, as long as an individual has his/her personal/social information, thoughts, emotions, feelings and problems, the person may needs to share his/her thoughts, emotions, and feelings with others as well as all kinds of personal information, news, private issues, experiences and so on. How and with whom a person shares such personal information embodies trust (Butler, 1999). A relationship between trust and information sharing is explained very succinctly by a study conducted by Kerr, Stattin and Trost (1999), in which a positive relationship between trust and disclosure of information was found. Indeed, the sharing of personal information with others is another typical domain in which to investigate the trust circle in this study.

Money is a domain in which people can equate value, and it does not encompass other utilities. Generally speaking, probably, there is no good money or bad money in the world. However, in people's personal life, the information people tend to trust and share with others is usually either "good" (positive) or "bad" (negative), so this study will 
examine both positive and negative personal domains in personal information sharing domain. Niu (1995) found that elementary students in China chose different trustees to share their personal information with, according to the type of information or emotions they wanted to share. Cvetkovich, Siegrist, Murray and Tragesser (2002) conducted a study in which they assessed the influence of trust on the effects of good and bad news, they found that the influence of good or bad news differs: those low in general trust judged bad news as more informative than good news than those high in general trust judged. Because there are so many varied things people need to share and those things are not neutral but rather are positive or negative, can we study the domain-based trust circle using only positive (or negative) information and then generalize to all other situations? The answer is no.

It could be expected that people's trust circle in the personal/social domain will not be exactly the same in both positive and negative settings. In this study, I will categorize shared personal information into positive (i.e. good news, pleasant experiences, happy stories and so on) and negative (such as bad news, unpleasant experiences, sad stories, frustrations, problems, etc.) in order to better explore the nature of the trust circle. This makes the personal domain different from the economic risk domain, since there are two positive and negative valences of personal information sharing domain, but no further valence in economic risk domain.

In the positive or negative personal/social domain, the levels of risk in entrusting information differ. For negative information, the issue can vary from a light unpleasant experience to extremely bad news. For positive information, the issue can vary from a 
simple exchange of information to boasting or bragging. This distinction, about anticipated outcomes of trusting someone, is of particular interest in this study because it may affect the size and composition of the trust circle, and further, may show cross cultural differences.

Trust as a reciprocal relation If there are two parties involved in a transaction, whether it involves financial or personal matters, the person who places trust in the other must believe that the other is trustworthy; the person also expects and may receive the other's trust at the same time. People develop attributions about their own trustworthiness, which in turn affect their willingness to accept trusting behavior from others. That is, trust is a reciprocal behavior: "I trust others" and "others trust me". I believe that trust in the "I-trust-others" direction and the "others-trust-me" direction may differ, the study uses locus of trust to describe the two trust states, "I trust others" and "others trust me", because the trust sender and trust receiver differ in the two trust loci, specifically, the trust sender in the locus of "I-trust-others" changes to trust receiver in the locus of "others-trust-me", and the trust receiver in the locus of "I-trust-others" becomes the trust sender in the locus of "others-trust-me". The relationship between the locus of trust and the trust circle will be explored and the concept of trust circle is useful to explore these differences.

In short, the domain (financial and personal), the valence of the domain (positive and negative personal information), the level of risk involved (from mild to serious), and the locus of trust ("I-trust-others" and "others-trust-me") are all issues to be explored in this thesis. The additional question involves the cultural comparison, is now addressed below. 


\section{The Trust Circle in Different Cultures}

"Cultures are seen as products of past human behavior and as shapers of future human behavior. Thus, humans are producers of culture and, at the same time, our behavior is influenced by it" (Segall, 1999, p.23). That is, all human behavior is cultural to some extent because the human species is fundamentally a social one (Berry, Poortinga, Segall \& Dasen, 2002c). Human behavior is meaningful only when viewed in the socio-cultural context in which it occurs (Segall, 1979).

Berry (1974) pointed out the inappropriateness of importing some American social-psychological concepts into Canada, such as studying French Canadian-English Canadian relations using an implicit model derived from African American-White American research. People in any given cultural context gradually develop a set of social behaviors that enables them to function well - naturally, flexibly, and adaptively - in a range of situations, and these situations are fairly common and recurrent in the cultural context (Kitayama, Markus, Matsumoto \& Norasakkunkit, 1997). There are relationships between context variables and psychological variables across different cultures (Berry, 2000). As a kind of social behavior and a psychological tendency, trust is a constituent element of a given cultural system and cannot be separated from it.

Kitayama et al. (1997) assert that an individual's psychological systems must be attuned to and coordinated with the cultural system in which he or she is. Here lies a clear and important opinion that culture and the mind/consciousness of people make each other up. A study about people's psyche (such as mind, awareness, consciousness etc.) should also include their cultural background if a whole picture is expected. Many studies have examined the interaction in a wider scope, such as culture and self-concept (Mastlund, 
Norlander \& Archer, 2001), culture and cognitive style (Engelbrecht \& Natzel, 1997), culture and temperament (Brandstrom, Richter \& Przybeck, 2002), culture and attachment (Ditommaso, Brannen \& Burgess, 2005), culture and self-esteem (Phelps, Taylor \& Gerard, 2001; Zhang, 2005) and so on. As the product of certain cultures, the trust circle should be studied with its cultural background. Since trust and the involved culture are part of each other, separating them in research must give incomplete results. By exploring generalities and specifics of the trust circle in cultural contexts, we can better understand the fundamental features of trust.

The question of cultural differences addresses a more general issue: at what level are there similarities across cultures (universals), and where are there important specific differences? One can see that basic behaviors and basic psychological processes are likely to be common features to all members of the human species; however, culture influences the development and display of psychological characteristics, that is, culture engenders different variations on these underlying themes (Berry et al., 2002c).

Cross-cultural studies give us both culture-general and culture-specific knowledge (Triandis, 1980). As long as people understand the culture-specific trust issue, people can understand culture-general trust better and vice versa. The relationship between culture in the widest sense and psychological phenomenon is the central issue (Jahoda, 1980). It is possible, in the domain considered for this thesis, that cultural differences in the trust circle may shed light on how features of the two cultures influence trusting.

Generally speaking, a functional approach to conducting cultural research is to compare different cultures, thus requiring at least two cultures. People usually try to find cultures that differ from each other as much as possible to serve the cross-cultural study 
purpose. Why Canada and China were chosen for the cultural sample in the study has been mainly discussed previously. The two nations represent two distinct cultures of the West and the East. From a geographical point of view, the two countries are far from each other. China is in the Eastern hemisphere, and Canada is in the Western hemisphere. In addition, the countries' histories, political systems, organizational systems, languages, traditions, lifestyles, etc are very different. In terms of cultural background, Canada and China are meaningful, well-functioning participants in their unique cultural environments. They have gradually developed a particular set of psychological processes and social behaviors that are attuned to and therefore support and match the prevalent patterns of the cultural systems.

Many scholars have demonstrated a number of differences between Asia and the West (Kashima, 2002; Kashima \& Triandis, 1986; Nisbett, Peng, Choi \& Norenzayan, 2001; Triandis et al., 1988). For example, Kitayama et al. (1997) believe that Western cultures are organized according to meanings and practices that encourage individual independence and autonomy of a self that is separate from other similar selves and from social context. On the other hand, many Asian cultures do not highlight the separation of each individual, in contrast, these cultures are organized according to meanings and practices that encourage a fundamental connectedness among individuals with a significant relationship (e.g. family, workplace, and classroom). Individualism, as a cultural pattern, is found in most western regions, including North America, while collectivism is common in Asia (Triandis, et al., 1988).

The construct of individualism and collectivism (I-C) is very wide ranging, multifaceted, and possibly over-extended to the point that it has become a catch-all to 
explain a very large number of psychological differences across cultures (Kagitcibasi, 1997). It is obvious that individualism/collectivism has long been of significance in social thought about human nature and the relationship among human beings. The promise of individualism/collectivism is "perhaps the most important dimension of cultural difference in social behavior across the diverse cultures of the world" (Triandis, 1988, p.60).

Contemporary Canadian culture, in contrast with contemporary Chinese culture, highlights the importance and necessity of making personal choices, forming judgments, and having opinions of one's own. Becoming a well-functioning member in this cultural context requires that one possess a set of psychological resources that generate and keep accessible a wide array of individual attitudes and personal preferences (Kitayama et al., 1997). In individualist countries, everyone is expected to look after him or herself. The opposite of an individualistic culture is a collectivist one. In collectivist countries, people are integrated into strong, cohesive ingroups from birth (Porter, 1997).

The defining difference between individualism and collectivism is a primary concern for oneself in contrast to a concern for the groups(s) to which one belongs. For example, Triandis (1995) proposes that there are four defining attitudes: 1. the definition of the self as personal or collective, independent or interdependent; 2. personal goals having priority over group goals (or vice versa); 3 emphasis on exchange rather than on communal relationships; and 4. relative importance of personal attitudes versus social norms in a person's behavior.

According to general ideas about individualism and collectivism, it is believed that in Eastern countries or collectivist cultures, such as China and Korea, family plays a 
central role; however, in Western countries or individualist cultures (i.e. North America), there is less emphasis on family associations (Porter, 1997). Unlike Western culture, Chinese culture is often noted for its particularism and insistence on building up trust through cultivating personal relationships (Trompenaars, 1993).

However, some scholars disagree with the idea that the East and the West represent collectivism and individualism separately, because the issue is not a simple one. Some scholars argue that Chinese/Asians represent individualism and that Canada/West represents collectivism. For example, Fijineman, Willemsen \& Poortinga (1996) carried out an experiment to test some predictions about differences in behavior between societies previously characterized as collectivist or individualist in Hong Kong, Turkey, Greece, the United States and the Netherlands. They found that emotional closeness to specific social others (e.g., father, sister, cousin, close friend, neighbor, unknown person) explained differences in inputs and outputs, rather than being due to the more general variable of individual-collectivism in all six countries.

Regarding levels of trust among individualist and collectivist countries, such as the United States and China, there have emerged some contradictory conclusions. Fukuyama (1995) suggests that the United States has a higher degree of generalized trust and spontaneous sociability than does China. Also, Fukuyama's concept of the trust boundary depends on the strength of the social ties in relationships. According to Fukuyama, the Chinese trust boundary is quite close-in, accepting only kin to the inner circle, while in the United States, this boundary is much farther out, including individuals outside of one's family. 
Buchan and Croson (2004) tested Fukuyama's ideas by examining reported levels of trust and trustworthiness extended to and expected from various family members (parent, sibling, cousin) and non-family members (student you know well, student from another university, stranger from your home town, stranger from another country) among American and Chinese participants. The study was conducted as an investment game with participants being randomly assigned to roles of proposer and responder. They found higher levels of reported generalized trust in China than in the United States. They also failed to demonstrate a strict boundary of trust in China among kin-related counterparts. Actually, they believed that there is no significant difference between the boundaries of trust among Chinese and Americans.

Based on the data collected in the 1990 wave of the World Values Survey (Inglehart, Basanez \& Moreno, 1998), although levels of general trust between family and non-family for both China and the US fall markedly, levels of trust in the US toward strangers remained relatively high, while those in China dropped. However, Buchan and Croson (2004) did not see a more significant drop in trust among Chinese participants between the family and non-family counterparts than they saw for American participants.

These contradictory results concerning the link between cultures and trust leave us with few conclusive findings and the issue of national differences in trust and trustworthiness is one that we as psychological researchers need to better understand.

Thus, the research project of this thesis is to explore these questions through the conceptual framework of the trust circle. The research allows an important comparison of two cultures in which it might be expected that the trust circle might differ in ways relevant to the cultural differences signified above. 


\section{PURPOSE and RESEARCH DESIGN}

This study is an exploratory study because little is known about the trust circle and there seems to be no similar research to be used as a point of reference.

Given the purpose of the study, exploring the trust circle in both economic and personal domains, I decided to use the "lending of money", an economic domain involving risk, as a basis from which to create a questionnaire because money permeates our world and perhaps embodies trust more than many other behaviors in the current world. A decision about whether or not to lend money to others is based on whether or not the others are trusted and how they are trusted. If there is no trust at all, the reasonable consequence may be that no money is lent, and how you trust others is reflected by how much money you choose to lend to others. Similarly, the economic decision to initiate a risky transaction, such as an internet purchase, involves trust, as does a decision to invest money in a business etc. In all these cases, there is an element of risk of personal harm, as well as personal gain.

I chose "sharing" as another basis from which to create a questionnaire in the personal domain since in this world we live, telling others one's news, stories, or experiences is a very common interaction between people and no doubt also embodies trust because "trust assumes a key position within this transactional process of information exchange or communicative interaction" (Thiede, 2005, p.1452). In contrast to the neutrality of money, personal information has either a positive or negative nature for individuals. As mentioned before, it would be indispensable to explore both contents of the domain in order to understand the trust circle in all its aspects. Also, there is a risk 
involved in engaging in personal domain, such as being jealous by others in sharing positive information with others, reputation being discredited in sharing negative personal information.

The next issue is how to design the study in order to best explore trust circle size and composition in economic risk and personal information sharing domains. Buchan and Croson (2004) believe that an intentional question is more highly correlated with actual behavior than the more attitudinal measure (general question). Why the results of studies conducted by the 1990 World Values Survey (WVS) and a study conducted by Buchan and Croson (2004) are contradictory is related to the measuring issue. In the WVS, the trust question is only generally asked, such as how much can you trust other people? As the result, the study found that Chinese demonstrated less trust overall than did Americans and were much less trusting of people from other countries. On the other hand, in Buchan and Croson's study, specific behavioral intention is elicited, that is in a given situation, the participants were asked how much money they would send or return to their partner. The study shows that there is a higher level of reported trust amongst the Chinese than amongst the Americans.

Some of contradictory results in studies of trust may be partly due to the fact that some researchers emphasize generalized trust in non-specified context, while others investigate trust using specific questions/contexts. The concept of the trust circle makes it possible to suggest that trust may differ, according to responses to several set of specific questionnaires. Responding to "do you trust your friend to lend $\$ 500$ to him/her" versus "how do you trust your friend" would get different results. This study will measure the trust circle size and trust circle composition with specific referents in order to get more 
correlated data. For instance, in the economic domain, participants will be asked "whom, do you think, you trust to lend $\$ X$ to, if possible?" A list of potential trustees will be provided for the participants to choose from and a set amount of money arranged from a small amount to a larger amount.

In addition, each domain will be expressed at 4 different risk levels/degrees to provide a varied domain to examine whether the trust circle differs as the level of domain differs, giving us a opportunity to observe the developmental tendency of the trust circle.

A pilot study was conducted to obtain information about domains of trust, categories of people who are trusted, and levels of perceived risk in trusting others. The pilot study is reported below.

\section{PILOT STUDY}

Many trust studies have been conducted without preliminary work to establish the meanings of the categories that will be employed in the research. For example, in their research examining cross-cultural differences in relationships and group-based trust, Yuki et al. (2005) used 3 scenarios to provide a variety of situations. Participants were asked to first read the scenarios and then indicate the extent to which they would trust specific types of strangers in those situations; the scenarios were created without a pilot study. Buchan and Croson (2004) explored the boundaries of trust in the United States and China with an investment game. In the study, a list of counterparts was provided with increasing social distance order. The 7 types of counterparts were: parent, sibling, cousin, student you know well, student from another university, stranger from home town, 
stranger from another country, and this list was also created without a pilot study. The authors did use prior research to establish their categories, but no evidence was provided that these categories would be meaningful to the people in the group they wished to study.

This trust circle study could also been conducted without a pilot study but I preferred to conduct one because this is a new area of study exploring a new idea. The main purpose of the pilot study was to confirm and provide basic and general information in order to help create an original and appropriate questionnaire.

\section{Sample in Pilot Study}

Thirty-six participants participated in the pilot study, including 19 Canadians and 17 Chinese, half were males, and half females. All Canadians and the majority of Chinese were recruited from psychology 1002 courses at Carleton University. A few of the Chinese participants were recruited "accidentally" or by getting people known to the researcher, since there were not enough Chinese who were registered in the Psychology course 1002 and were also interested in participating in the study.

\section{Procedure in the Pilot Study}

The first aim of the pilot study was to obtain the following information:

1. A possible list of trustees people would like to extend trust to.

2. Minimum and maximum amounts of money people would trust to lend to others, if possible meaning if they had that much money.

3. Some common positive and negative personal information people usually trust and share with others. 
In the economic risk situation, the corresponding questions were thus prepared: "what would be the possible minimum and maximum amounts of money you think you would trust to lend to others, if you had such money?" and "whom do you think you would trust to lend the minimum amount of money to?" In the personal information sharing domain, questions were as follows: "what kinds of positive/negative information (i.e. good/bad news, happy/sad stories, pleasant/unpleasant experiences, etc.) would you trust and share with others?" and "whom would you trust to share the best/worst positive/negative information with?"

Participants were met individually at their convenience and responded to the above questions. All questions were open and participants were free to respond to the questions. Participants were also encouraged to list as many things as they could.

\section{Questionnaire Construction}

The second purpose of the pilot study was to create a set of questionnaires to be used in the main study.

Based on the data obtained from the pilot study (free responses to the open questions, discussions with participants, supervision from my supervisor, my own thinking, as well as the aims and feasibility of the study), an original questionnaire was created (see Appendix A, Appendix B, Appendix C, Appendix D, Appendix E, Appendix F, Appendix G, Appendix H, Appendix I, Appendix J, Appendix K, Appendix L).

After frequency, sorting, and analysis, the following list of potential trustees was created to provide to the participants. The following trustee list would be kept the same for each and every question and in all situations. An important consideration was the frequency with which certain categories of people appeared on the list of trustees. While 
trying not to lose important information, and also not to create a clumsy list, I combined some trustees based on commonality and the overall theoretical framework of the trust circle. For example, husband, wife, boyfriend, girlfriend, partner, lover, are combined as one item, being close personal relationships; I also separated some trustees according to their distinguishing characteristics, for example, best/close friend is one category while friend is another since the function and role of best/close friend is different from that of common friend (Kenrick \& Trost, 2000).

\begin{tabular}{|c|c|c|c|}
\hline Acquaintance /Neighbor & 1 & Grandparents & 8 \\
\hline Bank manager/Accountant & 2 & $\begin{array}{l}\text { Husband (wife) /Boyfriend } \\
\text { (girlfriend) /Partner /Lover }\end{array}$ & 9 \\
\hline Best/close friend & 3 & Parents /Guardian & 10 \\
\hline Boss /Supervisor /Teacher & 4 & Psychologist /Professional counselor & 11 \\
\hline Children /Grandchildren & 5 & Relative & 12 \\
\hline Classmate/Co-worker/Colleague & 6 & Sibling & 13 \\
\hline Friend & 7 & Stranger & 14 \\
\hline
\end{tabular}

The trustee list is made distinctively and fully because it comes from people, not just from the researcher's own imagination and those trustees who overlap have already been combined into one item; in addition, the participants' freedom to suggest ensures as wide a scope of trustees as possible and is more effective than a result taken for granted. In order to examine what the trust circle might look like when the risk degree of economic domain changes, several levels of financial risk are needed, so a range of $\$ 10$, $\$ 500, \$ 10,000$ as well as $\$ 100,000 \&$ more was chosen as the 4 levels of money lending risk based on the results from the participants' responses in the pilot study. I tried to make each economic level significantly different from the next level; for instance, 
choosing $\$ 10, \$ 20, \$ 30, \$ 40$ as the 4 levels of financial risk would not provide much difference between levels. Thus 4 questions were asked and each question aimed at each economic risk level/variant with a total of 4 levels: $\$ 10, \$ 500, \$ 10,000, \$ 100,000 \&$ more.

In the Chinese version, the 4 financial risk levels correspondingly are $¥ 10$, $¥ 500$, $¥ 10,000, ¥ 100,000$ and more. If we use market exchange rates, $¥ 10$ obviously does not equal \$10. However, if we use the idea of Purchasing Power Parity (P.P.P.), which is necessary when comparing standards of living between countries, since an important part of a family's expenditures is on locally produced goods and especially labor services, the actual purchasing power of $¥ 10$ is roughly equal to $\$ 10$, based on buying routine goods such as eggs, bread, coffee, rice etc (Cooper, 2005).

Almost everyone, generally speaking, regardless of whether they are university students or not, has some kind of educational experience, personal information related to education may therefore be more common than other experiences. This expectation was confirmed by the responses in the pilot study. Thus the personal information was chosen from the most often mentioned education background, and in both positive and negative valence.

Based on the following three criteria, the 4 levels/variants of the positive and negative personal information were chosen.

First of all, they should differ from each other, for example, failing a course and failing a program were not felt to be very different by some participants because, sometimes, failing a course implies failing a program, thus I combined the two items into one. 
Secondly, the levels should be in a suitable range; for example, if being the best student in school/university is chosen, it would be meaningless to choose being best student in the city, in the nation, since people usually already trust and share the positive information (best student in your school/university) as much as possible, they may not go any further

Thirdly, the situation should be one which is meaningful in both cultures; for example, in the face of two possible choices (if you had good comments on your assignment /homework and if you had good comments on your presentation), the former is chosen since in China students hardly get the chance to make presentations, but they do have assignments/homework quite often, like Canadians students.

Therefore, the 4-levels/variants of positive personal information chosen were: 1 . if you had good comments on your assignment/homework; 2. if you got a good mark/grade on your test/examination; 3 . if you were the best student in your class; 4 . if you were the best student in your school/university.

In order to compare and analyze both the positive and negative personal information sharing situations, I tried to parallel the 4-levels of the negative personal information with the four levels of the positive personal information: 1. if you had bad comments on your assignment/homework; 2. if you got a bad mark/grade on your test/examination; 3. if you failed your course/program; 4. if you were expelled from your school/university. It was initially intended that these four variants should be parallel to the four levels of risk presented in the economic domain, but there was no way, in the present study, to calibrate the levels of risk exactly. Nor was it possible to design parallel domains of risk from the personal domain to the economic domain. However, since the 
pilot study indicated that respondents differentiated the transmission of positive vs. negative information to a trustee, this distinction was used in the materials subsequently developed for the main study.

All questionnaires were in both English and Chinese, the Chinese version being created by me, since my first language is Chinese. All Chinese versions were double checked by a professor of English at Yunnan Normal University in China. The Canadian participants were given the English version and the Chinese participants were given the Chinese version.

\section{MAIN STUDY}

The study explores the size and composition of the trust circle in two domains (economic and personal). Within the personal domain, there were two sets of questions, one concerning positive and one concerning negative information sharing It was initially intended that the economic and personal domains would each represent increasing levels of risk of entrustment. Economic, or financial risk was represented in terms of money entrusted; risk in the personal domain was represented in terms of positive and negative information about the self. This produced a design that was not balanced, but given the exploratory nature of the study, it seemed to be a reasonable way to proceed.

\section{Method}

\section{Participants}

There is a sample bias problem that is common in studies of social psychological phenomena. Frequently, participants are first year psychology students. In theory, the 
ideal sample should contain people of all kinds if the results of the experiment or survey are to apply to all people. There is strength in this argument because, in many important ways, university students do not differ from the population at large, although they are younger, more educated, and likely to come from a higher socio-economic group.

However, can the results of a study using only university students as participants be applied to all people? The answer is a wider sample is desirable, particularly when the study is about cultural differences and variables which may change in value across the life span (e.g. existence of a spouse, children, and so on). In order to deal with the problem of a restricted sample, or to reduce the bias of the sample, Yamagishi \& Yamagishi (1994) used two types of samples, one being a student sample and the other being a general population sample.

The sample for this dissertation will be comprised of two samples. One will be university students and the other will be gathered from the general population off-campus and will include various age groups, education levels, economic levels, occupations and so on. Thus what the study explores is not only the trust of college educated students but also the trust of common people with a sampling of males and females aged 18-77, which represents a broad spectrum of levels of social and economic status within each culture.

In the study, there were 202 volunteer participants, 101 male and 101 female. The average age was 32.44 with a median age of 22.00 . One hundred and two of the 202 participants were Canadian (50 male and 52 female) with a mean age of 31.94 . The other 100 participants were Chinese (50 male and 50 female) with a mean age of 32.92 . In both Canadian and Chinese groups, half were university students and the other half were drawn from the general population. 
For the university student sample, the 52 (25 male, 27 female) Canadian students, with mean age 20.23, were from Carleton University in Ottawa, Canada. They took introductory psychology courses and were recruited within the campus; for their participation, they received a credit in a scheme for recruiting participants, which translated into one percentage point of their overall grade (maximum grade possible, $100 \%$ ). The 50 Chinese students (25 male, and 25 female, mean age 19.34) were from Yunnan Normal University (foreign language major), Kunming Science and Technology University (computer science major) and Kunming Poly-Technical College (law major) in Southwest China; they all volunteered, when asked by their instructor if they would be interested in participating.

For the sample from the general population, the sampling criteria were devised to ensure diversity in age, gender and education/occupation Thus, the Chinese participants (male 25, female 25, mean age 46.50) in Kunming were recruited in a public service, a driving school, and a residence centre. Similar criteria were used for the Canadian sample. For this part of the Canadian group, 50 people (male 27, female 23, mean age 44.12) were recruited from parents at Glebe Collegiate, a community board, an apartment building, and two staff members at Carleton University. All participants from the general population were volunteers.

To choose the sample in mainland China, it was important to ensure that participants could be correctly described as Chinese. This is difficult to demonstrate empirically, but as a native Chinese speaker from Kunming, I was confident that all of the respondents regard themselves as Chinese. In China, there are few ethnic groups or immigrants; the people who participated in the study were native Chinese speakers. 
During the Canadian sample recruitment, I made sure to avoid international students; in addition, through the question "how many years have you lived in Canada", no Canadian participant had lived in Canada less than 3 years (permanent residents who live in Canada for 3 years can apply to become Canadian citizens). In light of the fact that Canada is an immigrant country, Canadians come from many different countries, but the dominant culture is Anglo-Canadian, and the sample was comprised of people who identified themselves as "Canadian".

\section{Procedure}

Canadian students individually completed the questionnaire on computer in the lab with my guidance. Chinese university students completed their questionnaires on paper in 3 groups in classrooms at the 3 universities with my guidance after their usual class was over.

The Canadian non-student participants individually completed their questionnaires either on paper or by computer, and were guided by me. The Chinese general population participants were organized in small groups of 6-7 people and answered the questionnaire in these groups with my assistance.

During the procedure, I paid attention to several research controls: controlling for understanding, it is important that participants understood the required task. Because the principal researcher is bilingual in Chinese and English, it was possible to assess level of comprehension, and to assist people who may had difficulty with the task. General instructions were provided at the top of each questionnaire, and the detailed instructions were presented for each question thus controlling for experimenter effects, to insure that the research was carried out in a similar manner in each country. While it would have 
been desirable to have multiple researchers to ensure inter-tester reliability, resources were not sufficient to allow this.

There are 4 sections presented in this discussion of the main study. The first deals with the trust circle in the economic risk domain, while the second concerns the trust circle in the personal domain. The third part compares the trust circle between the two domains. The final section deals with trust loci (I trust others and others trust me). In each of these, cross-cultural data will be considered. While it is not a central aim of this thesis to examine differences of gender, age, etc., however, when this is relevant, these data will be presented.

\section{TRUST CIRCLE in the ECONOMIC RISK DOMAIN}

The results of this exploratory study about the trust circle in the economic domain are expected to answer these questions:

1. whether the trust circle and trust composition are changeable as the level of economic risk varies and, if so, how do they change? What are the regularities?

2. whether different Western-Eastern cultures affect trust circle sizes and trust circle composition, if the trust circle is influenced by culture, how Canadians' trust circle differs from Chinese's trust circle.

In the pilot study, the following set of questions was used in the economic domain part, with 4 different economic risk levels, (lending $\$ 10, \$ 500, \$ 10,000, \$ 100,000 \&$ more to others). These levels were chosen to represent increasing risk levels to the trustee. 
The question asked: "whom do you think you would trust to lend $\$ 10$ to, if possible? Choose possible person(s) from the following list, and place an $\mathrm{X}$ within the corresponding small box". The words "if possible" dealt with the issue, which arose in pilot testing, that people might not be in a position to lend large amounts of money.

\begin{tabular}{|l|l|l|l|}
\hline Acquaintance & 1 & Grandparents & 8 \\
\hline Bank manager/Accountant & 2 & $\begin{array}{r}\text { Husband (wife)/Boyfriend } \\
\text { (girlfriend)/Partner/Lover }\end{array}$ & 9 \\
\hline Best/close friend & 3 & Parents/Guardian & 10 \\
\hline Boss/Supervisor/Teacher & 4 & Psychologist /Professional counselor & 11 \\
\hline Children/Grandchildren & 5 & Relative & 12 \\
\hline Classmate/Co-worker/Colleague & 6 & Sibling & 13 \\
\hline Friend & 7 & Stranger & 14 \\
\hline
\end{tabular}

It was possible for people to check none, one, some or all of the options; the larger number of people included is an index of a larger trust circle size. The 14 categories came from the free response data in the pilot study as mentioned previously. The groups of people included in the list checked by respondents represent "composition" of the trust circle. Since it was not known, a priori, whether respondents would group people into any particular categories (e.g. family, friends, professionals), several examples of each of these were provided.

Trust circle size and trust circle composition in the economic risk domain will be discussed first, followed by the results relevant to cultural differences in the trust circle size and composition. Then I will present data on the relationship between the trust circle and age, gender, education level and family income to see whether these have an influence on the trust circle. 


\section{Results and Discussion}

\section{Trust Circle Size in the Economic Risk Domain}

Circle size is reflected by the numbers of people nominated by respondents as people they would be willing to lend money (of different amounts) to. As Figure 1 shows, the average chosen number of trustees whom people would lend $\$ 10$ to was $9.76(S D=$ 3.45 ), the average number of trustees whom people would lend $\$ 500$ to decreased to 6.50 $(S D=2.59)$, the average trustee number for lending $\$ 10,000$ was much lower $(4.55, S D=$ 2.32), and the chosen trustee mean for lending $\$ 100,000 \&$ more was the lowest of all $(3.59, S D=2.95)$.

Figure 1: Mean Trustee Numbers at the 4 Economic Risk Levels

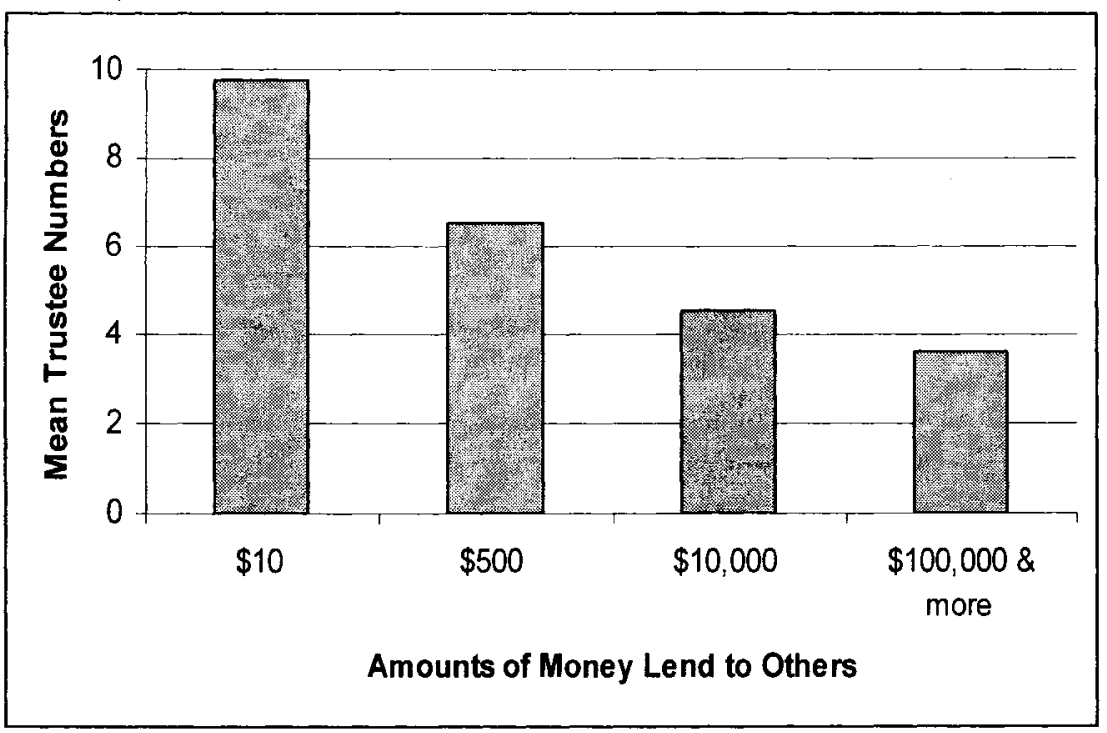

After an initial ANOVA across the four levels of risk showed a significant effect, post-hoc comparisons on the pairwise differences were conducted. It showed that trustees were increasingly less willing to lend money to others, trustees between lending $\$ 10$ to others and lending $\$ 500$ to others are significant differences $(t(201)=17.91, p<.001)$, 
the difference of trustees between lending $\$ 500$ and lending $\$ 10,000$ is also significant $(t(201)=14.67, p<.001)$, and trustees between lending $\$ 10,000$ and lending $\$ 100,000 \&$ more, too $(t(201)=7.10, p<.001)$.

Trust is important and necessary in risky situations and would not be needed if actions could be undertaken with complete certainty and without risk (Lewis \& Weigert, 1985; Hardin, 2001; Yamagishi \& Yamagishi, 1994). There is agreement by most researchers on the condition that risk must exist for trust to arise. Risk is one condition considered essential in psychological, sociological, and economic conceptualizations of trust (Rotter, 1967; Williamson, 1993; Yamagishi \& Yamagishi, 1994).

The results confirm and also develop the previous knowledge with the indication that increasing risk is associated with the range of people one would trust; as risk increases, the average chosen trustee number decreases and the trust circle size therefore becomes smaller. Thus, it is likely that when dealing with a small amount of money, say $\$ 10$, the risk is correspondingly smallest, the trustor would trust a largest number of trustees; when the money lent to others is increased to $\$ 500$, people may think about whether they believe that the trustee is worthy of their confidence, not everyone who is trusted with a $\$ 10$ loan can be trusted with a $\$ 500$ loan, and thus, some of the trustees are excluded from the smaller trust circle. When the money lent is increased to $\$ 10,000$, people are more likely to think whether they will continue to trust the same set of people they trust with a $\$ 500$ loan. When a new situation is riskier and, as a result, the number of trustees decreases, the trust circle then becomes smaller, and so on.

Trust circle size explains an important attribute-trust width, how many people are trusted, and also mirrors the level of trust. In the study, the four different risk levels of 
lending money correspond to different chosen trustee number means, or each level of risk in the economic domain matches its own circle size, and the trust circles vary according to the different levels of economic risk. Larger amounts of money lent correspond to smaller average trustee numbers; on the contrary, smaller amounts of money lent correspond to larger average trustee numbers. As the cost of default increases, the willingness to trust decreases.

\section{Trust Circle Composition in the Economic Risk Domain}

In addition to size, composition is another component of the trust circle. Who will be in the trust circle? What kinds of relationship exist for the focal person? Do those trustees change when the financial risk changes?

For analysis of composition of the trust circle, I used a summary measure, which used the frequency of people who ticked a particular person from the list, as someone they would trust at the four levels of lending. The rank of 1 was given if most participants chose that trustee, the rank of 2 if this was the second most chosen trustee, and so on. The average rank represents as index of the hierarchy of trusting particular others in the trust circle. Also, the rank is based on the proportion, for example shown in Table 1, proportion .96 means that $96 \%$ of participants tick best/close friend to lend $\$ 10$ to, since it is the highest choosing proportion, so the corresponding rank is 1 , sibling is chosen by $89 \%$ of participants to trust in lending $\$ 10$ to, the matching rank is 2 , and the proportion is .89 .

As can be seen, the ranks and proportions varying of the 14 trustees in the 4 economic risk levels are shown in Table 1. If the mean proportion of each possible trustee 
varies, then the rank varies. Majority trustees' ranks in the Table 1 change, and some stay the same across the levels of risk

Table 1: Ranks and Proportions of Trustees at the 4 Economic Risk Levels

\begin{tabular}{|c|c|c|c|c|c|c|c|c|}
\hline & \multicolumn{2}{|c|}{$\begin{array}{c}\$ 10 \\
(N=202)\end{array}$} & \multicolumn{2}{|c|}{$\begin{array}{c}\$ 500 \\
(\mathrm{~N}=202)\end{array}$} & \multicolumn{2}{|c|}{$\begin{array}{l}\$ 10,000 \\
(\mathrm{~N}=202)\end{array}$} & \multicolumn{2}{|c|}{$\begin{array}{c}\$ 100,000 \\
\& \text { more } \\
(\mathrm{N}=202)\end{array}$} \\
\hline & Rank & Prop & Rank & Prop & Rank & Prop & Rank & Prop \\
\hline Best/close friend & 1 & .96 & 1 & .85 & 4 & .59 & 5 & .37 \\
\hline Sibling & 2 & .89 & 3 & .82 & 2 & .69 & 3 & .50 \\
\hline Parents/Guardian & 3 & .87 & 2 & .84 & 1 & .77 & 1 & .70 \\
\hline Relative & 4 & .87 & 6 & .60 & 7 & .32 & 7 & .15 \\
\hline Husband(wife) /Boyfriend & 5 & .81 & 4 & .79 & 3 & .67 & 2 & .53 \\
\hline Friend & 6 & .78 & 9 & .31 & 10 & .10 & 10 & .05 \\
\hline Classmate /Co-worker / & 7 & .73 & 10 & .28 & 11 & .09 & 11 & .04 \\
\hline Grandparents & 8 & .73 & 5 & .69 & 5 & .56 & 4 & .39 \\
\hline Boss/Supervisor/Teacher & 9 & .65 & 8 & .33 & 8 & .16 & 8 & .09 \\
\hline Acquaintance/Neighbor & 10 & .64 & 11 & .24 & 12 & .08 & 12 & .03 \\
\hline Children/Grandchildren & 11 & .55 & 7 & .40 & 6 & .34 & 6 & .24 \\
\hline Bank manager/Accountant & 12 & .38 & 12 & .17 & 9 & .12 & 9 & .09 \\
\hline Psychologist / Counselor & 13 & .36 & 13 & .08 & 13 & .02 & 13 & .02 \\
\hline Stranger & 14 & .18 & 14 & .00 & 14 & .00 & 14 & .00 \\
\hline
\end{tabular}

Generally speaking, each chosen proportion of trustee tends to decrease gradually as the money lent increases, so the ranks vary based on the corresponding proportion comparing other trustees in the same economic risk level.

In detail, most people would lend a small amount of money to almost all of the categories of others, most people would only lend larger amount of money to few of the categories of others, and there are 3 following changing styles. 
1. Some trustees rank change from a higher rank (or high chosen proportion) to a lower rank (or lower chosen proportion), they are best/close friend (from rank 1 to rank 4 and finally to rank 5), relative (from rank 4 to 6 to 7 ), friend (from rank 4 to 6 to 7 ), classmate /co-worker/colleague, and acquaintance/neighbor. In the descending tendency, most of these types of people are outside of the family circle and thus, to some people, they are seen as outsiders.

2. On the contrary, some trustees choosing proportions change from a lower rank to a higher rank; this may suggest that trustworthiness of those trustees increases when the amount of money lent goes up; they are parents/guardian (from rank 3 to 1), spouse /partner (from rank 5 to 4, 3 and 2), grandparents, children /grandchildren and bank manager/accountant. In the ascending tendency, most of the trustees are family members with the exception of bank manager/accountant. In the fourth level (lending $\$ 100,000 \&$ more), the top 7 ranks are all family members except for best/close friend (rank 5).

3. At the same time, some trustees keep almost the same rank regardless of the amount of money lent: they are sibling, boss/supervisor/teacher, psychologist /professional counselor, and stranger. In addition, several types of persons (psychologist/professional counselor, and stranger) are always located in the last ranks therefore showing no changes in rank.

One can see that, in addition to the chosen trustee numbers gradually diminishing as the amount of money lent increases, people's choice changes correspondingly (Table 1). For example, best/close friend was ranked first in lending $\$ 10$ and $\$ 500$, but was less preferred (ranked $5^{\text {th }}$ ) in lending $\$ 100,000 \&$ more. This suggests that most people trust their best/close friend with a loan of $\$ 10$ and $\$ 500$, while when faced with larger 
financial risk, people's trust in them decreases to the fifth rank. Siblings seem to be trusted across all levels of risk with almost the same rank. Parents /guardians, in contrast, are trusted increasingly as the amounts of lending money increase.

Moving from the risk level of lending $\$ 500$ to the highest risk of lending 100,000 $\&$ more, the trustees ranked in the top 7 are all family members (Table 1), with the exception of best/close friend. It is the family which brings a person into the world and takes care of him/her, the financial link between a person and his or her family continues through a whole lifetime. Families always tend to provide unconditional financial support to their members as much as possible. Compared with people who are outside the family, in the crucial financial situation, people's tendency to trust family members seems more understandable (Clarke, Heaton, Israelsen \& Eggett, 2005).

It is a very interesting finding that close/best friend is trusted most and thus ranks first in lending $\$ 10$ and $\$ 500$. In the lending $\$ 10,000$ domain, best/close friend is still trusted, following only parents/guardian, sibling, and spouse /partner/lover. Even in the highest risk economic domain, people still trust best/close friend more than 2 family members, children/grandchildren and relative.

The position of best/close friend among all potential trustees is really unique and important. Several researchers have particularly studied the best friend relationship (Endo, Heine \& Lehman, 2000; Guttmann, 1993; Sakai, Sugawara, Maeshiro, Sugawara \& Kitamura, 2002), and a study conducted by Endo et al. (2000) in particular investigated the equal/close relationship between best friend and family members or a romantic partner. One could say that best/close friend is the most trustworthy trustee among all other out-of-family trustees and is even trusted the same as some family members based 
on our results. I cautiously propose that the composition of best/close friend seems to be equal with family member in people's minds, or in other words, best /close friend is in the core of the trust circle like family members in the economic risk domain. I will continue to examine the role of best/close friend in the personal domain to see if it behaves similarly.

In summary, as the risk of the economic domain increases, the people listed as trustees alters. That is, the hierarchy of trustees chosen in different economic levels is different: at the lower economic risk level, the hierarchy and relationship for the focal person are best/close friend, sibling, parents/guardian, relative, spouse/partner, friend and classmate/co-worker in the first seven ranks; at the higher economic risk level, the composition of the trustees changes to parents/guardian, spouse/partner, sibling, grandparents, best/close friend, children/grandchildren, and relative in the first seven ranks. Best/close friend is still the top trustee among non-family trustees even though the rank decreases to number 5, and among family trustees, parents /guardian rank number one.

\section{Cultural Effects on Trust Circle Size in the Economic Risk Domain}

A aim of this study was to examine whether the trust circle differs in different cultures, which differ in social structures that may shape people's beliefs about who is, and is not, trustworthy. We will first examine trust circle size in the Canadian and Chinese samples, followed by a consideration of the composition of the trust circle in each country.

When comparing the two cultures' trust circle size, a general circle size of each culture is needed, thus an average trust circle size mean is used. Figure 2 indicates that 
when Canadians and Chinese lend only $\$ 10$ to others, the Canadian chosen trustee mean number is 10.05 and the Chinese chosen trustee mean number is 9.47 , showing no significant differences between the 2 trustee numbers with repeated measures analysis $(F(1,200)=1.45, p>.05)$. However, as the amount of lending money increases to $\$ 500$, the Canadian trustee mean number decreases sharply to 5.95 while the Chinese trustee mean number, though it also decreases, decreases less than the Canadian sample, to 7.07. The two trustee means of the Canadians and the Chinese (5.95 vs. 7.07) are significantly different $(F(1,200)=9.91, p<0.01)$, and the Chinese seem to trust a larger number of people than do the Canadians. This pattern continues when the amount of money lent increases to $\$ 10,000$, the numbers of people trusted by Canadians and Chinese are 4.01 and 5.08 respectively $(F(1,200)=10.84, p<0.001)$. The trustee average numbers of Canadians (2.89) and Chinese (4.30) show a significant difference $(F(1,200)=12.17, p<$ 0.001 ) when the amount of money lent goes up to $\$ 100,000 \&$ more.

Figure 2: Mean Trustee Numbers of Canadians and Chinese at the 4 Economic Risk Levels 


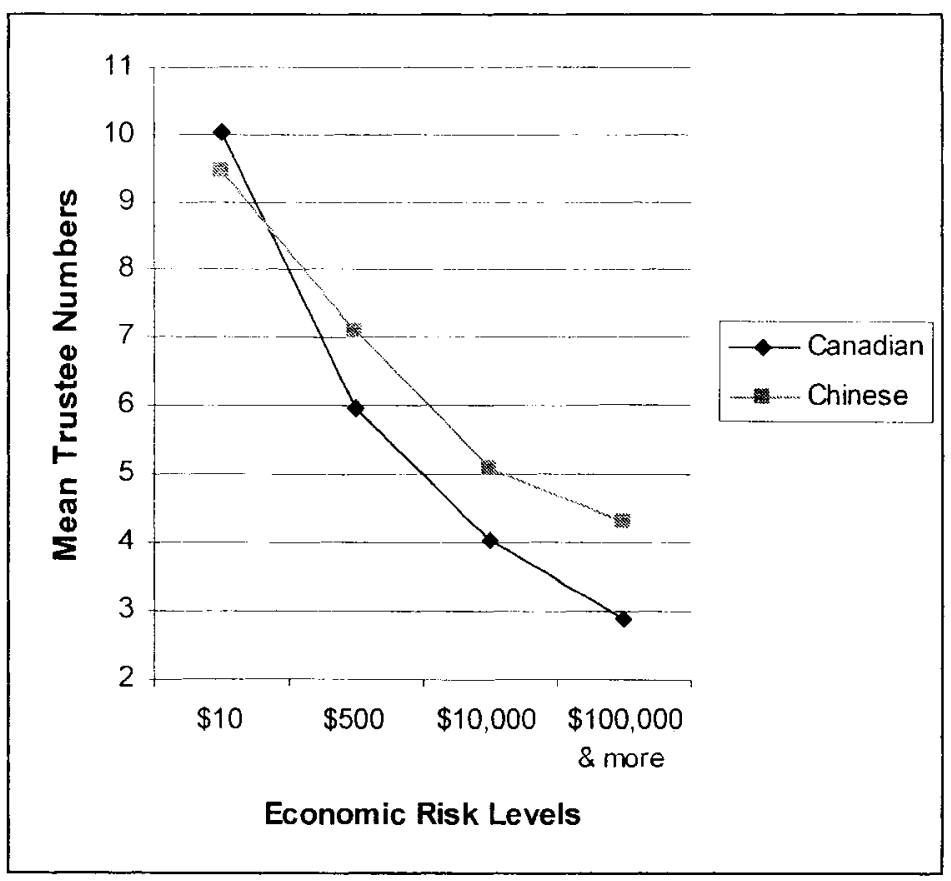

That is, when the financial risk is smaller, there is no real difference in the trust circle size in both cultures but when the economic risk becomes higher, cultural differences appear. The higher the economic risk, the larger the cultural difference with the Chinese tending to trust a larger number of other people than Canadians and the analyses of repeated measure found that culture affects trust circle size significantly $(F$ $(1,196)=11.92, p<.001)$

To control for differences across participants that could influence results, variables identifying age, education, family income, and participant status (student or non-student) were added as covariates to the analyses of repeated measure, and then it was found that culture affects trust circle size significantly, $F(1,196)=11.92, p<.001$.

Compared to Canada, China is still a developing country; the financial levels of majority are not very high, and the general population's income is still comparatively lower than it is in Canada. Thus, in general, Chinese may have a more difficult time in dealing with financial issues, so lending money to each other happens more often in 
China. Furthermore, when people face with some major/serious financial need, such as going abroad, getting sick, marrying, losing a family member, etc, they tend to seek support from their family members, friends, and those who have previously lent money from them (Logan \& Bian, 2003; Pei \& Pillai, 1999). Lending and borrowing money is a relatively common part of Chinese life, compared with Canadians, Chinese are more familiar with this economic practice.

One possible reason for Chinese's reliance on family and friends in meeting economic need is that members of the general population in China have a more difficult time getting loans from the government or from banks than Canadians do, even when they desperately need money in critical times. The most likely approach to overcome a time of need in China is to borrow money from other people. In other words, financial support is probably more easily obtained from banks or from the government in Canada (Ontario Ministry of Training, Colleges and Universities, 2005; Zhang, 2005). Therefore, the idea of lending and borrowing money is not a rare thing in China and the Chinese are fairly comfortable with the idea. This is not to say that all Canadians have access to institutional assistance, but it is more available in Canada than in China.

Moreover, in China, people usually pay more attention to relationships or guanxi (Chow \& Ng, 2004; Farh et al., 1998; Hwang, 1997). If someone asks to borrow money from you, you probably feel that they trust you and, if you have the money, you usually lend it to them to keep a good relationship. Furthermore, when you lend money to someone, it is seen as a favor, the borrower remembers this and may returns it at a later time in some other way. This is also an action for future possible benefits from others. It seems that Chinese Guanxi contributes to a larger trust circle size. Chinese culture 
dictates that people should rely on other people's financial support in critical times. Thus Chinese people have a long tradition of such financial behavior which may result in them trusting a larger number of people even for comparatively large amounts of money.

On the other hand, in Canada, it seems common in the culture to avoid lending or borrowing money from private individuals. In general, Canadians seem more likely to follow the rule of "Never Lend Money, Car and Wife" as I have heard. Canadians tend to be more independent, which includes financial independence (Kerr, Normand, 1992; Watkins, Mortazavi \& Troffmova, 2000). Lending or borrowing money may not be considered as independent behavior, let alone when a larger amount of money is involved, and the availability of financial support from non-personal sources in Canada contributes to each individual's perceived independence.

Finally, Figure 2 shows that both Canadians and Chinese tend to hesitate trusting many others as the economic risk becomes greater. Generalizations and similarities across cultures could be expected due to our single-species heritage and the necessity of adapting to environmental constraints (Munroe \& Munroe, 1997); certain features may be common to all cultures. On the other hand, as long as the involved cultures differ, the trust, as a product of culture, will display some unique/specific features. That is, when the economic domain becomes riskier, Chinese seem to trust more others than Canadians do but, when the economic situation is not risky, both Canadians and Chinese trust identically.

\section{Cultural Effects on Trust Circle Composition in the Economic Risk Domain}

According to the results of the study (Figure 3), one can clearly recognize the trust circle composition differences between Canadians and Chinese. First of all, in the 
financial risk domain, when faced with lending money to someone, no trustees were trusted exactly the same by both Chinese and Canadians. The majority of trustee choices vary significantly between Canadian and Chinese participants, meaning that the trust circle composition differs across cultures.

Figure 3: Fourteen Trustee Proportions of Canadians and Chinese in the Economic Risk Domain

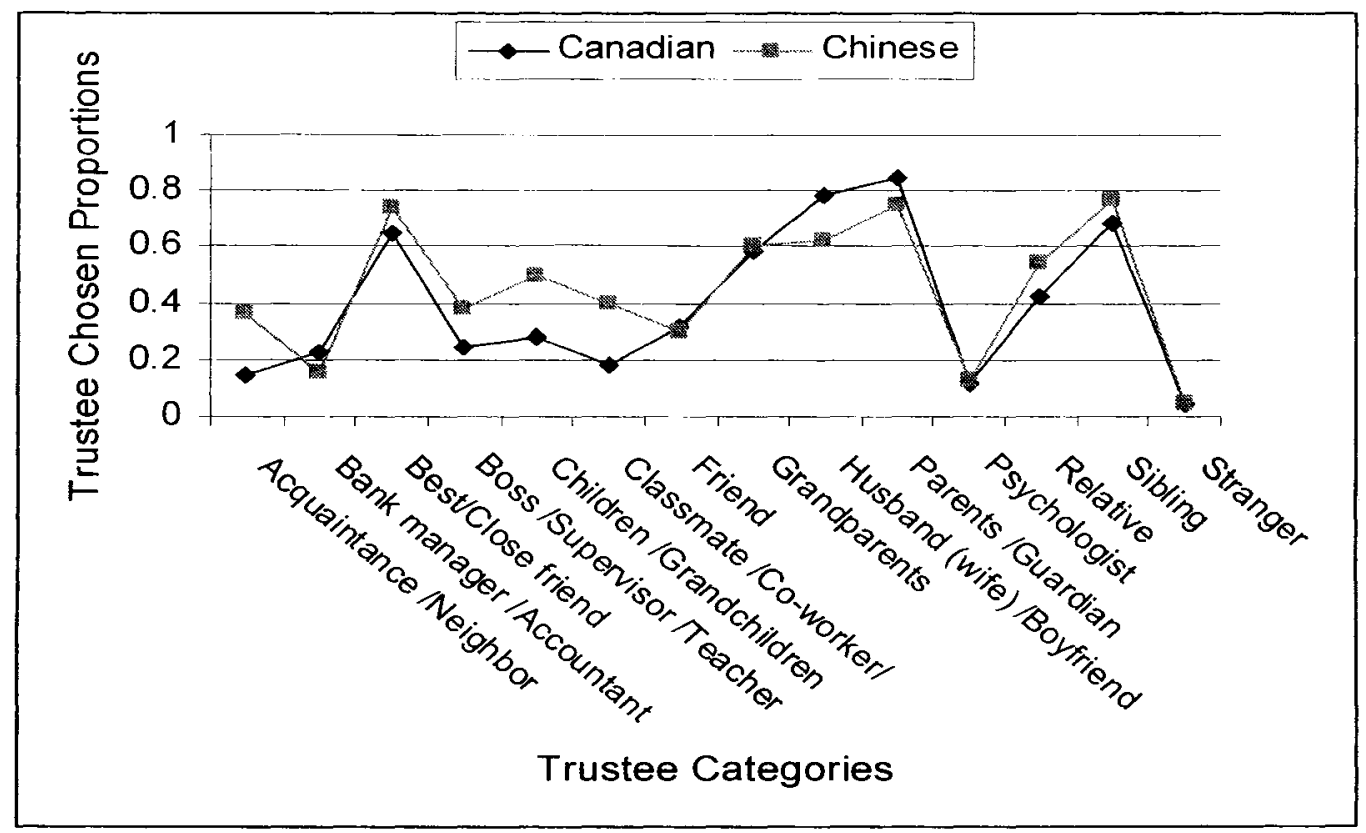

In Figure 3, although majority trustee categories are trusted more by Chinese than by Canadians, Canadians are likely to trust spouse/partner and parents/guardian more than Chinese do and the difference in the trust of the spouse/partner is most outstanding.

I would like to stress the trust difference of "spouse/partner of family vs. other members of family" at this point. Based on personal experience and observation, as well as communications with Canadians, I realize that in Canada the spousal relationship is the most intimate relationship in both the material life and the mental world. Canadians view the spouse/partner as the most important relationship to the exclusion of all others unlike 
Chinese. All other family relationships may break down or be temporary, for example, one could separate from one's parents when one is 18 years old and become a totally independent individual, and one could also separate from their children when their children are 18 years old. Similar circumstances happen to other family members. The longest lasting relationship most Canadians have may be with their spouse/partner, rather than with any others; the position of spouse/partner in Canadians' mind is superior to that of other family members.

However, in China, even though the spousal relationship is also important within the family, it is not particularly emphasized. In traditional Chinese culture, sometimes priority is given to other family members rather than spouse and it is every Chinese's unshakable duty to provide support to older and younger generations (Pei \& Pillai, 2000). One can see from Chinese around or in the media etc. that often Chinese choose to pay the price of damaging their spousal relationship in order to maintain the interests of the whole family. To satisfy his/her parents, to raise sisters/brothers, to support relatives etc., a Chinese person may ignore or go against his/her spouse's interests or opinions (Jiang, Chaiwan, Panuthai, Yiang, Lei \& Li, 2002; Li \& Tracy, 2000).

The cultural differences in attitudes toward spouse/partner and other family members are reflected in the results. Canadians seem to trust the majority of trustees less than Chinese do, but Canadians seem to trust their spouse/partners more than Chinese do and the difference degree is at the top among family members.

Table 2 shows a hierarchy of trust of whom Canadians and Chinese trust most, whom will be the second to be trusted and so on in the economic risk domain. The rank is an average rank of 4 economic risk levels, and the means of proportion is the percentage 
of participants who have chosen the trustee. Among family members, Canadians trust parents/guardian most, then spouse/partner, followed by sibling, grandparents, relative, and lastly, children /grandchildren. Chinese first prefer to choose sibling, then parents /guardian etc, followed by spouse/partner and grand parents and so on. For non-family members, best/close friend is the first trustee in both cultures.

Table 2: Trustee Chosen Proportions and Standard Deviations of Canadians and Chinese in the Economic Risk Domain

\begin{tabular}{ccllcc}
\hline \multicolumn{3}{c}{ Canadian $(\mathrm{n}=102)$} & \multicolumn{3}{c}{ Chinese $(\mathrm{n}=100)$} \\
\hline SD. & Prop. & Trustee & Trustee & Prop. & SD. \\
\hline .26 & .84 & Parents /Guardian & Sibling & .77 & .30 \\
.28 & .78 & Spouse /Partner & Parents/Guardian & .75 & .36 \\
.33 & .68 & Sibling & Best/close friend & .74 & .30 \\
.25 & .65 & Best/close friend & Spouse /Partner & .62 & .37 \\
.37 & .59 & Grand parents & Grand parents & .61 & .38 \\
.27 & .42 & Relative & Relative & .54 & .30 \\
.22 & .32 & Friend & Children/Grandchildren & .49 & .40 \\
.33 & .28 & Children/Grandchildren & Classmate/Co-worker/ & .40 & .28 \\
.21 & .24 & Boss/ Supervisor /Teacher & Boss/ Supervisor/Teacher & .38 & .32 \\
.30 & .23 & Bank manager /Accountant & Acquaintance /Neighbor & .36 & .24 \\
.15 & .18 & Classmate/Co-worker/ & Friend & .30 & .24 \\
.12 & .14 & Acquaintance /Neighbor & Bank manager/Accountant & .16 & .27 \\
.17 & .12 & Psychologist/Counselor & Psychologist/Counselor & .13 & .20 \\
.09 & .04 & Stranger & Stranger & .05 & .10 \\
\hline
\end{tabular}

The above results indicate that the effects of culture on the composition of the trust circle are substantial. Those who are trusted most by the Chinese are not trusted in the same order by Canadians, and vise verse. Different cultures have their own favorite 
trustees. The majority of possible trustees are trusted differently. For example, the top 4 trustees for Canadians are parents /guardian, spouse/partner, sibling and best/close friend in proper order, while for Chinese, the top 4 trustees are sibling first, parent /guardian second, then best/close friend third followed by spouse/partner.

In order to explore the differences of trustee choices between Chinese and Canadians, I use discriminant analysis, since it is used to explore corresponding discriminates between two corresponding variables. As Table 3 shows, larger absolute discrimination values correspond to variables with greater discriminating ability and smaller absolute values correspond to variables with smaller discriminating ability. The positive numbers mean that Chinese trust more than Canadians and the negative numbers mean that Chinese trust less than Canadians. For example, the number -.24 in Table 3 tells us that Chinese are less likely to trust spouse /partner than Canadians, and even the discrimination number between Chinese and Canadians is in the fifth rank, it is the biggest difference with Canadian trust spouse/partner more than Chinese do, unlike the previous 4 trustees who all are trusted by Chinese more. At the same time, we can see the difference is significant at the $p<.001$ level.

Table 3: Discriminations of Trustees between Canadians and Chinese in the Economic Risk Domain 


\begin{tabular}{lccc}
\hline Trustees & $\begin{array}{c}\text { Discrimination } \\
(\mathrm{N}=202)\end{array}$ & $\mathrm{F}$ & Sig. \\
\hline Acquaintance /Neighbor & .55 & 64.01 & .000 \\
Classmate /Co-worker/Colleague & .47 & 47.07 & .000 \\
Children/Grandchildren & .29 & 17.86 & .000 \\
Boss/ Supervisor /Teacher & .26 & 13.94 & .000 \\
Spouse /Partner & -.24 & 12.26 & .001 \\
Relative & .20 & 8.74 & .003 \\
Best/close friend & .17 & 5.71 & .018 \\
Parents /Guardian & -.16 & 5.06 & .026 \\
Sibling & .14 & 3.83 & .052 \\
Bank manager/Accountant & -.12 & 3.04 & .083 \\
Friend & -.04 & .37 & .543 \\
Stranger & .03 & .17 & .667 \\
Grand parents & .03 & .13 & .717 \\
Psychologist /counselor & .02 & .08 & .777 \\
\hline
\end{tabular}

Table 3 also reveals that 9 of the 14 trustees chosen by Canadians and Chinese show significant differences, and the corresponding difference ranks are arranged from the biggest difference to the smallest difference. For those trustees Canadians trust more than Chinese do, the biggest discriminating trustees are spouse /partner among family members and bank manager /accountant among non-family members. For those trustees Chinese trust more than Canadians, the biggest discriminating trustee choices are acquaintance /neighbor among non-family members and children/grandchildren among family members.

The hierarchy of the trust circle is influenced by culture as shown with the ranks of trustees chosen by Canadians and Chinese. The first, second, third etc., core trustees of each culture differs and the majority of the differences between the 2 cultures are 
significant. Among them, the choice of spouse/partner showed the biggest difference between Canadians and Chinese with Canadians trusting spouse/partner more often than Chinese do; choosing acquaintance /neighbor showed the biggest difference between the 2 cultures by being trusted more by Chinese than by Canadians.

\section{Age, Education Level, Family Income, Gender, Participant Status and the Trust Circle in the Economic Risk Domain}

Gender, age, family income, and participant status (university student or common person) do not seem to contribute much to the trust circle in the economic risk domain. However, repeated measures analysis reveals that education levels (here the lower education level includes elementary school and high school; the higher education level includes college, university and graduate studies) do have an influence to the trust circle. It seems that people who have a lower education level tend to trust fewer others than those with higher levels of education (Figure 4), the difference being significant $(F(1$, $196)=19.44, p<.001)$. Also, people with higher level of education are likely to trust all 14 trustee categories more than people with lower education level (Figure 5); the difference between the two groups is also significant $(F(1,196)=19,98, p<.001)$.

Figure 4: Mean Trustee Numbers of People with Lower and Higher Levels of Education at the 4 Economic Risk Levels 


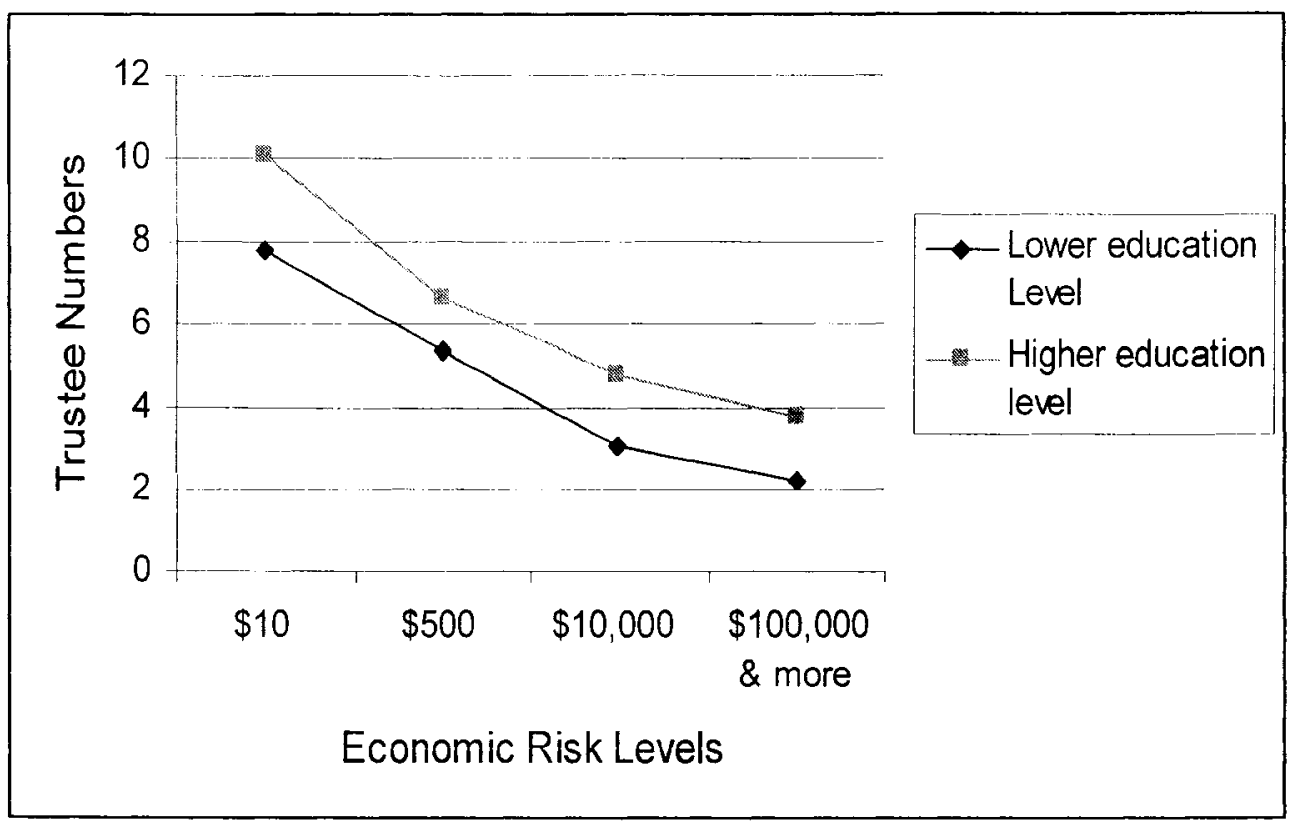

Figure 5: Fourteen Trustee Chosen Proportions of People with Lower and Higher Education Levels in the Economic Risk Domain

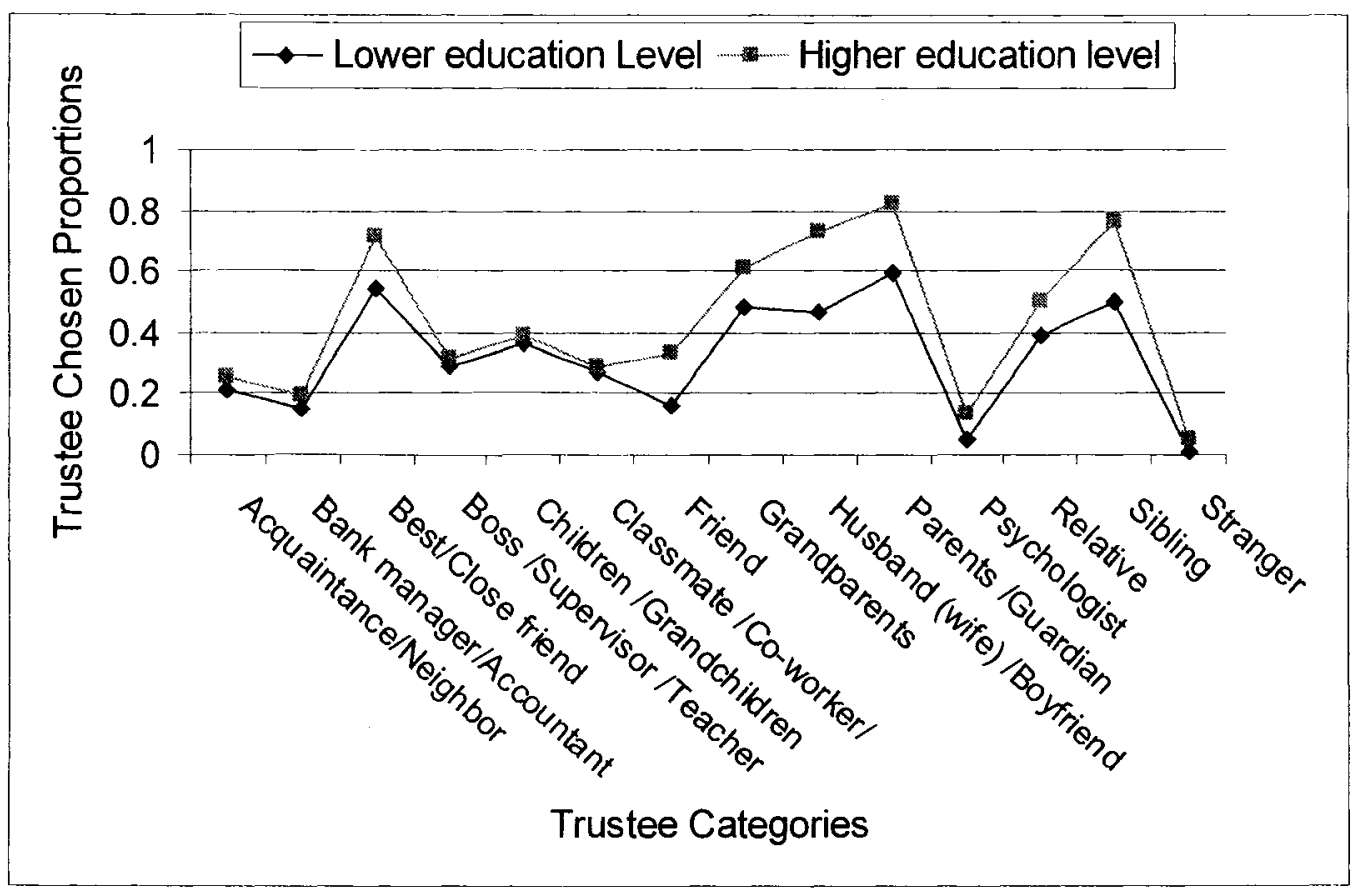


The results support the idea that higher education levels tend to be associated with trust, helpfulness etc. whereas lower education levels tend to be associated with distrust and selfishness etc. (Sares, 1998).

\section{Conclusion about the Trust Circle in the Economic Risk Domain}

Now, the 2 questions mentioned before can be answered based on the results of the trust circle exploring study in the economic domain.

1. The size of the trust circle, meaning how many trustees people would trust, is changeable, and it is affected by the level of risk of the economic domain. Different risk levels are associated with different circle sizes. Specifically, as the supposed lending money increases (the risk increases), the size of the trust circle decreases, meaning that fewer others are trusted, and vice versa.

2. The trust circle composition, meaning the relationship for the focal person and the hierarchy of trusting in the trust circle, is also changeable, and it also depends on the degree of risk of the economic domain. Different risk and demand situations are associated with different trust circle compositions. The ranks of family members tend to be higher than out-of-family members as the economic situation becomes more critical and risky; the ranks of non-family members are inverse.

3. Culture affects trust circle size and composition: Chinese tend to trust more others than Canadians do in higher economic risk, that is Chinese trust circle size tends to be larger than Canadians' trust circle size when the economic situation becomes riskier and more serious; Chinese trust a majority of others more than Canadians, however, a few trustees are trusted more by Canadians than Chinese, the biggest difference occurring with spouse/partner of family. 
4. Age, family income, gender and participants status do not cause differences in trust circle size and composition in the economic domain. However, education level does. More highly educated people seem to have a larger trust circle size and trust almost all trustee categories more than people with lower education level.

\section{TRUST CIRCLE in the PERSONAL INFORMATION SHARING DOMAIN}

The trust circle is also explored in the sharing personal information with others domain, corresponding to "sharing money" (lending to) with others. How likely people are to share their personal issues with others, as an important factor, reflects how likely people trust others in their personal/social life.

As mentioned before, the trust circle is examined in two different contents of personal information, positive and negative, because in real life, people's personal/social information tends to be either positive (e.g. good news, happy story, pleasant experience) or negative (e.g. bad news, sad story, unpleasant experience), thus, an integrated study should include the 2 contents of information. Furthermore, even in the same positive or negative personal information valences, they may differ on the level of positive or negative, from extremely good news to light pleasant, from a little upset to very sad punishment etc. Using these varying positive and negative information sharing variants, the trust circle was explored.

The aim of this part is to examine the size and composition of the trust circle in both positive and negative personal information sharing domains, as well as the cultural influences on the trust circle. Specifically, it is expected to find 
1. Whether the valence (positive and negative) of personal information affects the trust circle; 2. Whether the variant of positive and negative valence of personal information sharing domain influences the trust circle, and 3. Whether the trust circle is affected by culture, if so, how the valence, variant of valence and culture affect trust circle size and trust circle composition.

The section begins with trust circle size in the personal information sharing domain, continues with trust circle composition, then cultural effects on trust circle size are presented. After that, cultural effects on trust circle composition will be discussed and before the conclusion, the trust circle and other factors are presented.

\section{Results and Discussion}

Based on information from the pilot study, the following questions were used in the personal information sharing domain.

For the 4 variants of the positive personal information sharing domain:

1. If you had good comments on your assignment/homework, whom would you trust and share this information with? Choose possible person(s) from the following list and place an $\mathrm{X}$ within the corresponding small box.

\begin{tabular}{|l|l|l|l|}
\hline Acquaintance & 1 & Grandparents & 8 \\
\hline Bank manager/Accountant & 2 & $\begin{array}{r}\text { Husband (wife)/Boyfriend } \\
\text { (girlfriend) /Partner /Lover }\end{array}$ & 9 \\
\hline Best/close friend & 3 & Parents/Guardian & 10 \\
\hline Boss /Supervisor/Teacher & 4 & Psychologist /Professional counselor & 11 \\
\hline Children /Grandchildren & 5 & Relative & 12 \\
\hline Classmate/Co-worker/Colleague & 6 & Sibling & 13 \\
\hline Friend & 7 & Stranger & 14 \\
\hline
\end{tabular}


2. If you got a good mark/grade on your test/examination, whom would you trust and share this information with? The checklist is the same as the one used in question 1.

3. If you were the best student in your class, whom would you trust and share this information with? The checklist is the same as the one used in question 1.

4. If you were the best student in your school/university, whom_would you trust and share this information with? The checklist is the same as the one used in question 1.

For the 4 levels of negative personal information:

1. If you had bad comments on your assignment/homework, whom would you trust and share this information with? Choose possible person(s) from the following list and place an $\mathrm{X}$ within the corresponding small box.

\begin{tabular}{|l|l|l|l|}
\hline Acquaintance & 1 & Grandparents & 8 \\
\hline Bank manager/Accountant & 2 & $\begin{array}{r}\text { Husband (wife)/Boyfriend } \\
\text { (girlfriend)/Partner/Lover }\end{array}$ & 9 \\
\hline Best/close friend & 3 & Parents/Guardian & 10 \\
\hline Boss/Supervisor/Teacher & 4 & Psychologist/Professional counselor & 11 \\
\hline Children/Grandchildren & 5 & Relative & 12 \\
\hline Classmate/Co-worker/Colleague & 6 & Sibling & 13 \\
\hline Friend & 7 & Stranger & 14 \\
\hline
\end{tabular}

2. If you got a bad mark/grade on your test/examination, whom would you trust and share this information with? The checklist is the same as the one used in question 1.

3. If you failed your course/program, whom would you trust and share this information with? The checklist is the same as the one used in question 1. 
4. If you were expelled from your school/university, whom would you trust and share this information with? The checklist is the same as the one used in question 1.

\section{Trust Circle Size in the Personal Information Sharing Domain}

Figure 6 shows the average trust numbers of the 4 positive and negative levels of the personal information sharing domains. First of all, the trustee numbers look different between positive and negative information sharing domains with the number of trustees in sharing positive personal information being larger than the number of trustees in sharing negative personal information.

One can also see that in the sharing positive personal information domain, chosen trustee numbers tend to be different at the 4 levels (good comments on assignment, good mark/grade on test/exam, best student in class and best student in school/university). For sharing good comments on assignment information, the average trustee mean is 5.01 ( $S D$. $=3.96$ ), which is smaller than the average trustee mean of sharing good mark/grade on test/exam information $(5.99, S D .=3.70)$, and it is also smaller than the average mean of sharing the information (best student in class) $6.19(S D .=3.18)$. Among the 4 variants, the largest average trustee mean $(M=6.52, S D .=3.21)$ is in sharing best student in school/university information.

Figure 6: Mean Trustee Numbers of the 4 Positive (left) and Negative (right) Variants of the Personal Information Sharing Domain 


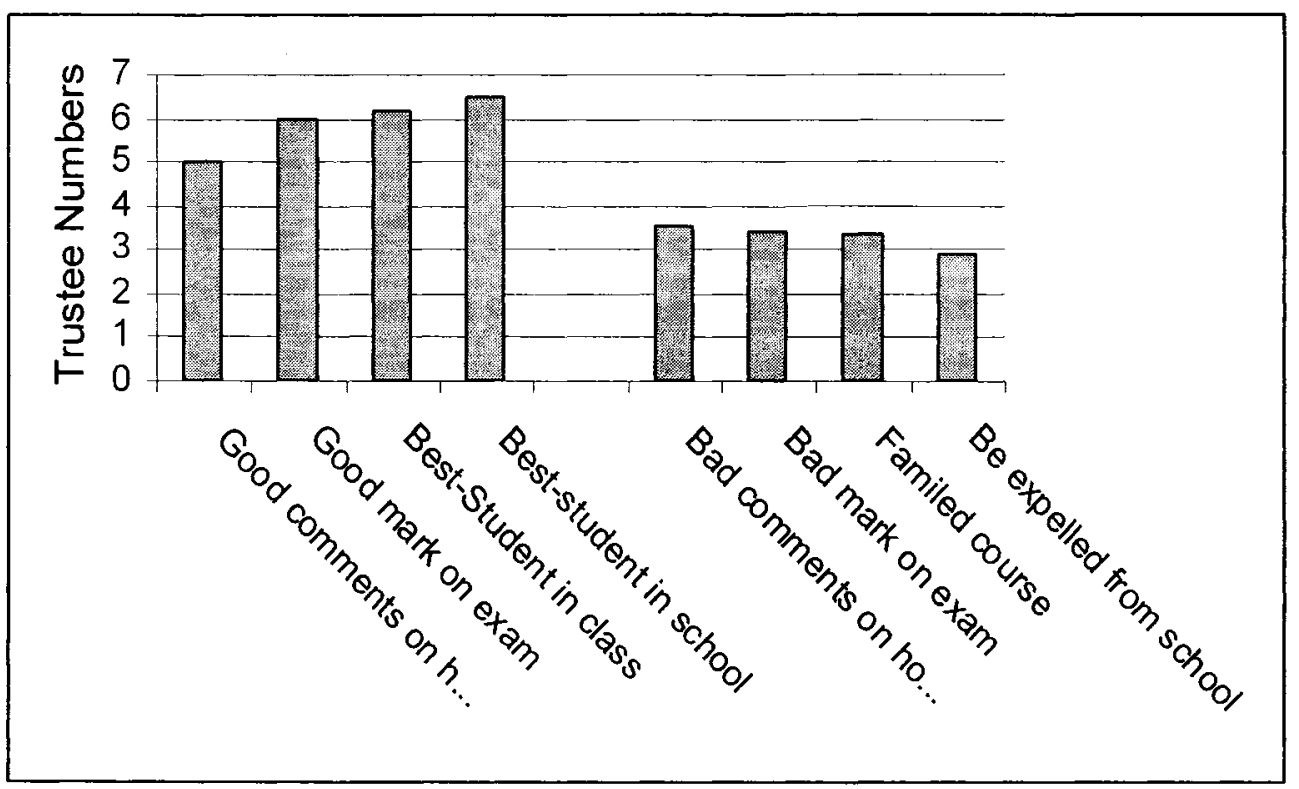

The developing pattern of the average trustee number means in the sharing positive information is based on how positive of the information. Generally speaking, the most positive information (if you were best student in your school/university) corresponds to a largest trustee number, and the least positive information (if you got good comments on your assignment) corresponds to the smallest trustee number.

The results demonstrate that the size of the trust circle is affected by how strong the positive personal information people would trust and share with others is. The trust circle size tends to increase as the positive level goes up. Indeed, people usually like to tell more people their best news, happiest experience and so on.

Similarly (Figure 6), in the sharing of negative personal information situation, choosing trustees also differs at the 4 different negative variants (bad comments on assignment, bad mark/grade on test/exam, failed course/program and expelled from school/university). For sharing bad comments on assignment, the average chosen trustee mean is $3.55(S D .=2.33)$, which is larger than the average mean in sharing bad mark/grade on test /exam, which is $3.43(S D .=2.22)$, and it is still larger than the average 
mean of sharing failed course/program $3.31(S D .=2.15)$. Among all 4 variants, the smallest chosen trust number mean $(M=2.87, S D .=1.70)$ is in sharing expelled from school/university information.

The most negative information (if you were expelled from your school/university) corresponds to the smallest average trustee mean, and the least negative information (if you got bad comments on your assignment) corresponds to the largest average chosen trustee number mean. It implies that the size of the trust circle is based on how negative the personal information people would trust and share with others is. In other words, the trust circle size tends to decrease as the negative information level goes up.

Moreover, as we can see in Figure 6, all the chosen trustee numbers in the 4 levels of positive personal information sharing are larger than all the chosen trustee numbers in the 4 negative levels of personal information-sharing. The average trustee number $5.92(S D .=3.08)$ of sharing positive information is different from the average trustee number $3.29(S D .=1.86)$ of sharing negative information by a significant margin $(t(201)=14.99, p<0.001)$. Indeed, the content of the shared personal/social information affects trust. Cvetkovich et al. (2002) conducted a study to explore the relationship between new information and social trust. They found that good news or bad news affected the level of trust, and people in low and high trust positions judged good/bad news differently.

In the study, people's trust circle size in the positive domain differs from their trust circle size in the negative domain with the trust circle size of sharing positive personal information being larger than that of sharing negative personal information. 
In summary, based on the valence (positive and negative) of the sharing personal information domain, trust circle size differs, a positive personal information-sharing domain is associated with larger trust circle size, and a negative personal informationsharing domain is associated with smaller trust circle size. In addition, trust circle size tends to be larger if the positive level of the shared personal information becomes stronger, and on the contrary, as the negative level of the shared personal information becomes stronger, the trust circle size tends to be smaller. In other words, the more positive the personal information is, the more others the people would like to share it with; the more negative the personal information is, the fewer others people would like to share it with.

\section{Trust Circle Composition in the Personal Information Sharing Domain}

As discussed above, trust circle size is the first variable of the trust circle and composition, which we will discuss here now, is the second one.

Looking at Table 4, when the shared personal information is positive, the first 5 trustee chosen ranks (best friend, parents/guardian, spouse/partner, sibling, and grandparents) stay the same hierarchically from positive variant 1 to positive variant 4 . The last 5 trustee ranks (children/grandchildren, acquaintance/neighbor, psychologist /counselor, bank manager, stranger) also keep the same hierarchy, with no one going up or down in the 4 positive levels. Only the trustees ranked in the middle (classmate, friend, relative, and boss) show some differences, such as classmate decreasing in ranking from 6 to 7 to 9 , and boss/supervisor/teacher increasing in ranking from 9 to 8 to 7 .

Table 4: Ranks and Proportions of Chosen Trustees at the Shared 4 Different Positive Information Variants 


\begin{tabular}{lcccccccc}
\hline Trustee & $\begin{array}{c}\text { Good } \\
\text { Comments on } \\
\text { Assignment } \\
(\mathrm{N}=202)\end{array}$ & $\begin{array}{c}\text { Good Mark } \\
\text { on Test } \\
(\mathrm{N}=202)\end{array}$ & $\begin{array}{c}\text { Best Student } \\
\text { in Class } \\
(\mathrm{N}=202)\end{array}$ & $\begin{array}{c}\text { Best Student } \\
\text { in School } \\
(\mathrm{N}=202)\end{array}$ \\
\cline { 2 - 9 } & $\mathrm{R}$ & $\mathrm{P}$ & $\mathrm{R}$ & $\mathrm{P}$ & $\mathrm{R}$ & $\mathrm{P}$ & $\mathrm{R}$ & $\mathrm{P}$ \\
\hline Best/close friend & 1 & .69 & 1 & .84 & 1 & .86 & 1 & .87 \\
Parents /Guardian & 2 & .66 & 2 & .80 & 2 & .81 & 2 & .83 \\
Husband (wife)/ & 3 & .64 & 3 & .76 & 3 & .74 & 3 & .77 \\
Sibling & 4 & .54 & 4 & .67 & 4 & .72 & 4 & .74 \\
Grandparents & 5 & .43 & 5 & .49 & 5 & .58 & 5 & .64 \\
Classmate /Colleague / & 6 & .41 & 7 & .43 & 9 & .34 & 9 & .35 \\
Friend & 7 & .36 & 8 & .43 & 7 & .40 & 8 & .42 \\
Relative & 8 & .31 & 6 & .44 & 6 & .51 & 6 & .54 \\
Boss /Supervisor/ & 9 & .25 & 9 & .31 & 8 & .38 & 7 & .46 \\
Children /Grandchildren & 10 & .25 & 10 & .29 & 10 & .30 & 10 & .31 \\
Acquaintance /Neighbor & 11 & .19 & 11 & .24 & 11 & .22 & 11 & .22 \\
Psychologist/Counselor & 12 & .15 & 12 & .15 & 12 & .18 & 12 & .20 \\
Bank manager/ & 13 & .07 & 13 & .10 & 13 & .09 & 13 & .10 \\
Stranger & 14 & .06 & 14 & .07 & 14 & .05 & 14 & .07 \\
\hline
\end{tabular}

Looking at the results, we see that 10 of the 14 trustees were chosen in the same important order, with no differences in the 4 variants of positive personal information sharing, the 10 trustees being either in the top ranks ( 5 of them) or in the bottom ranks ( 5 of them) and 4 of the 14 trustees being chosen in slightly different order. One may conclude that whom people trust to share their positive personal information with, generally speaking, does not change much at the different positive variants.

The trustee rankings show that in the positive personal information-sharing domain, best/close friend is the top favorite trustee or, in other words, people trust best/close friend much more than any other kind of trustee. After best/close friend, the 
following 4 trustees are all family members. Parents/guardian's trust order is higher than spouse/partner, which is higher than sibling which, in turn, is higher than grandparent. People tend to trust bank manager/accountant and stranger least.

Unlike sharing positive personal information, when sharing negative personal information, only the 2 top ranking trustees (best/close friend and spouse/partner) keep the same rank hierarchy through the 4 negative personal information variants (see Table 5) and only the 2 bottom ranking trustees (stranger and bank manager/accountant) are the same at the 4 different negative personal information sharing levels. The other 10 middle ranking trustees are ranked in different order at the 4 shared negative personal information variants. It seems that compared with sharing positive information, the hierarchy of trustees in sharing negative personal information is more diversified.

Table 5: Ranks and Chosen Proportions of Trustees at the 4 Different Negative Information Variants 


\begin{tabular}{lcccccccc}
\hline Trustee & $\begin{array}{c}\text { Bad Comments } \\
\text { on Assignment } \\
(\mathrm{N}=202)\end{array}$ & $\begin{array}{c}\text { Bad Mark } \\
\text { on Test } \\
(\mathrm{N}=202)\end{array}$ & $\begin{array}{c}\text { Failed Course } \\
(\mathrm{N}=202)\end{array}$ & $\begin{array}{c}\text { Expelled from } \\
\text { School } \\
(\mathrm{N}=202)\end{array}$ \\
\cline { 2 - 9 } & $\mathrm{R}$ & $\mathrm{P}$ & $\mathrm{R}$ & $\mathrm{P}$ & $\mathrm{R}$ & $\mathrm{P}$ & $\mathrm{R}$ & $\mathrm{P}$ \\
\hline Best/close friend & 1 & .75 & 1 & .76 & 1 & .76 & 1 & .68 \\
Spouse /Partner & 2 & .66 & 2 & .66 & 2 & .67 & 2 & .65 \\
Sibling & 3 & .43 & 3 & .44 & 4 & .40 & 4 & .38 \\
Parents /Guardian & 4 & .43 & 4 & .42 & 3 & .47 & 3 & .52 \\
Friend & 5 & .29 & 5 & .28 & 6 & .19 & 5 & .13 \\
Classmate /Colleague / & 6 & .25 & 6 & .21 & 5 & .19 & 9 & .07 \\
Grandparents & 7 & .17 & 7 & .14 & 7 & .13 & 6 & .11 \\
Relative & 8 & .13 & 10 & .11 & 9 & .11 & 8 & .08 \\
Psychologist /Counselor & 9 & .13 & 9 & .12 & 8 & .11 & 7 & .09 \\
Boss /Supervisor / & 10 & .12 & 8 & .12 & 10 & .10 & 11 & .04 \\
Acquaintance /Neighbor & 11 & .07 & 12 & .06 & 12 & .05 & 12 & .04 \\
Children /Grandchildren & 12 & .07 & 11 & .07 & 11 & .09 & 10 & .06 \\
Stranger & 13 & .03 & 13 & .03 & 13 & .02 & 13 & .02 \\
Bank manager / & 14 & .02 & 14 & .01 & 14 & .01 & 14 & .01 \\
\hline & & & & & & & & \\
\hline
\end{tabular}

Among the 14 potential trustees (Table 4 and Table 5), only best/close friend was ranked as the top choice to place trust in both positive and negative personal information sharing, and through all 4 variants, whereas other trustees' hierarchy of trusting is not stable in the two domains: for instance, parents /guardian was ranked in the second order to be trusted in all 4 positive levels of information sharing domain, but not in the negative domain.

Figure 7 shows the trustee chosen proportions in both positive and negative personal information sharing domains. First of all, the composition of the trust circles in the positive personal information sharing domain is different from the composition of the trust circle in the negative personal information sharing domain. Most proportions of all 
14 trustees chosen to be trusted sharing negative personal information are much fewer than the proportions in sharing positive personal information.

Figure 7: Proportions of 14 Trustees in the Positive and Negative Personal Information Sharing Domains

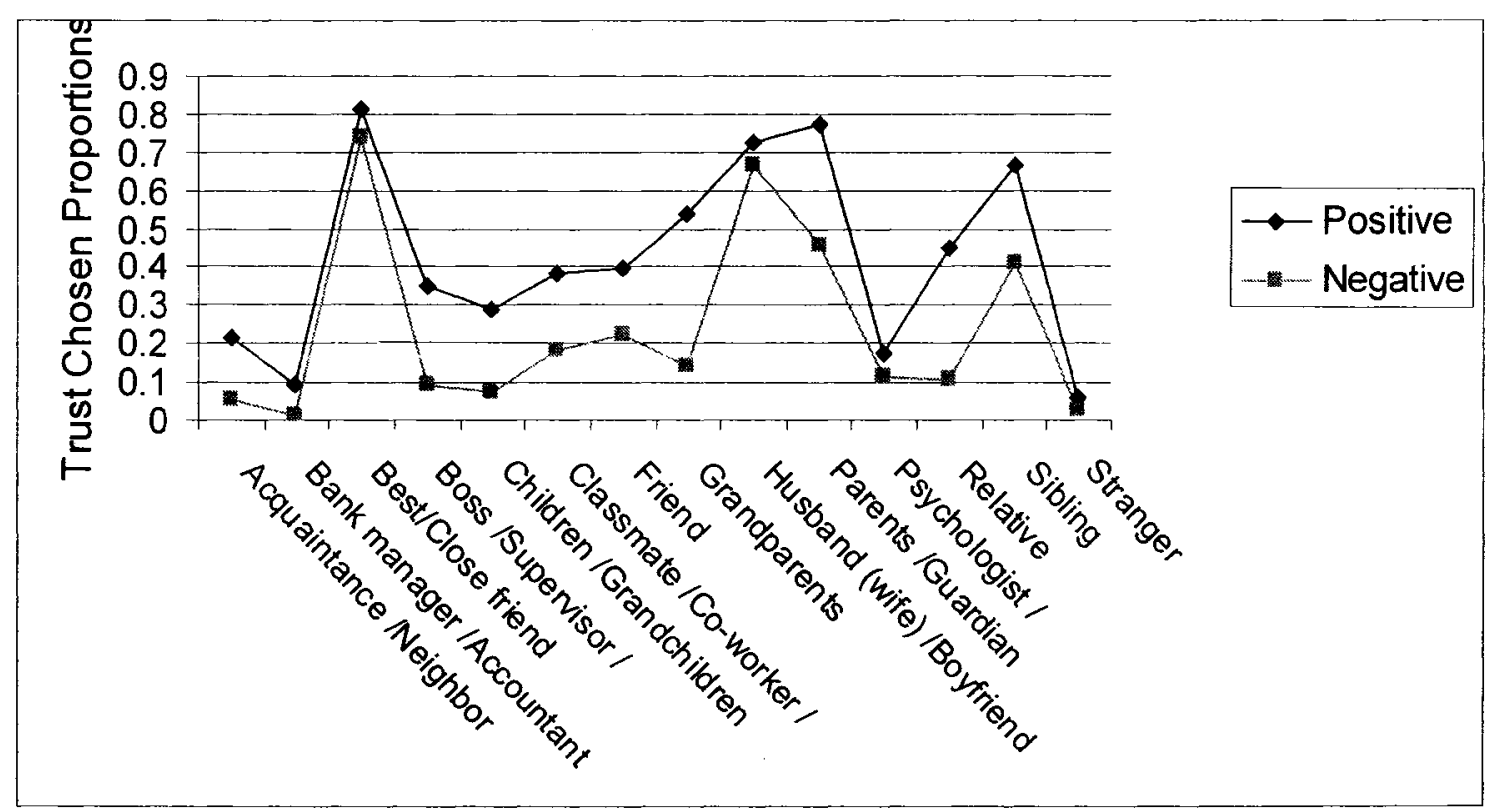

A repeat measure test indicates that chosen trustee proportions are significantly different between the two positive and negative personal information sharing domains ( $F$ $(1,201)=224.55, p<.001)$. It means that trustees chosen in certain domains differ in other domains; for example, $54 \%$ of people would trust and tell grandparents their good comments, good mark, good achievement etc., while only $14 \%$ of people would tell grandparents their bad comments, bad mark, bad achievement etc.; parents /guardian was ranked second in the positive domain but ranked in the $3^{\text {rd }}$ order in the negative domain, the ranking of spouse/partner is in the $3^{\text {rd }}$ order in the positive information sharing domain but in the $2^{\text {nd }}$ in the negative personal information sharing domain. 
The ranks of some family members, such as grandparents, relative, children /grandchildren, as well as boss supervisor/teacher are higher hierarchically when sharing positive personal information than when sharing negative personal information, meaning that they are trusted more in the positive information sharing domain than in the negative information sharing domain. On the contrary, some out-of-family trustees, such as friend, classmate/fellow student/co-worker/colleague, and psychologist/counselor are trusted more in the negative information sharing domain than in the positive information sharing domain.

The hierarchy of trusting, as well as relationships to the focal person in positive and negative information sharing domains change. The top 6 trustees in the positive personal information sharing domain are best/close friend, parents/guardian, spouse /partner, sibling, grandparents and relative separately. In sharing negative personal information situation, the top 6 trustees are best/close friend, spouse/partner, parents/guardian, sibling, friend and classmate /co-worker/colleague (Figure 7). In both domains, best/close friend is always in the top spot, parents /guardian in the second top rank in the positive domain and the third top rank in the negative domain, while spouse/partner, on the contrary, is in the third top rank in the positive and in the second top rank in the negative personal information sharing domain. Some extended family members (grandparents, relative, children /grandchildren) tend to be less trusted in the negative information sharing domain, some out-of-family trustees (friend, classmate/coworker colleague, psychologist /counselor) are more trusted but some out-of-family trustees (boss /supervisor /teacher, acquaintance) are trusted less in the negative information sharing domain. 
In order to further analyze the composition of the trust circle, I classify the 14 potential trustees into 4 categories: 1 . best/close friend, since it is dominant and it is distinct from all other trustees, also distinct from general peers as results of this study and idea of Rotenberg, Boulton and Fox (2005), 2. casual-relationship, including bank manager/accountant, psychologist /professional counselor, stranger, 3. friendship, including acquaintance /neighbor, boss/supervisor/teacher, classmate/fellow student/coworker /colleague, friend, and 4 kinship, which includes all family members: children /grandchildren, grandparents, spouse/partner parents/guardian, relative, sibling.

As we sea in Figure 8, people do not choose trustees randomly in both information sharing domains, meaning people particularly trust some more, trust some less, people usually pick the trustee they would like to trust and would like to share information with, since there is a big proportional difference between each of the 4 trustee categories chosen proportions. People choose best-friend to share both their positive and negative personal information with most often, then family members second. Following that, people prefer friend /classmate /acquaintance /neighbor etc. and those casual-relationship others (e.g. bank manage, accountant, psychologist) were chosen last.

The choosing differences of best-friend and family members, family members and friends, friends and casual relationships are all significant in sharing both positive and negative information (see Table 6 for results of post-hoc comparison measures).

Figure 8: Comparing Proportions of the 4 Trustee Categories Chosen in the Positive and Negative Personal Information Sharing Domains 


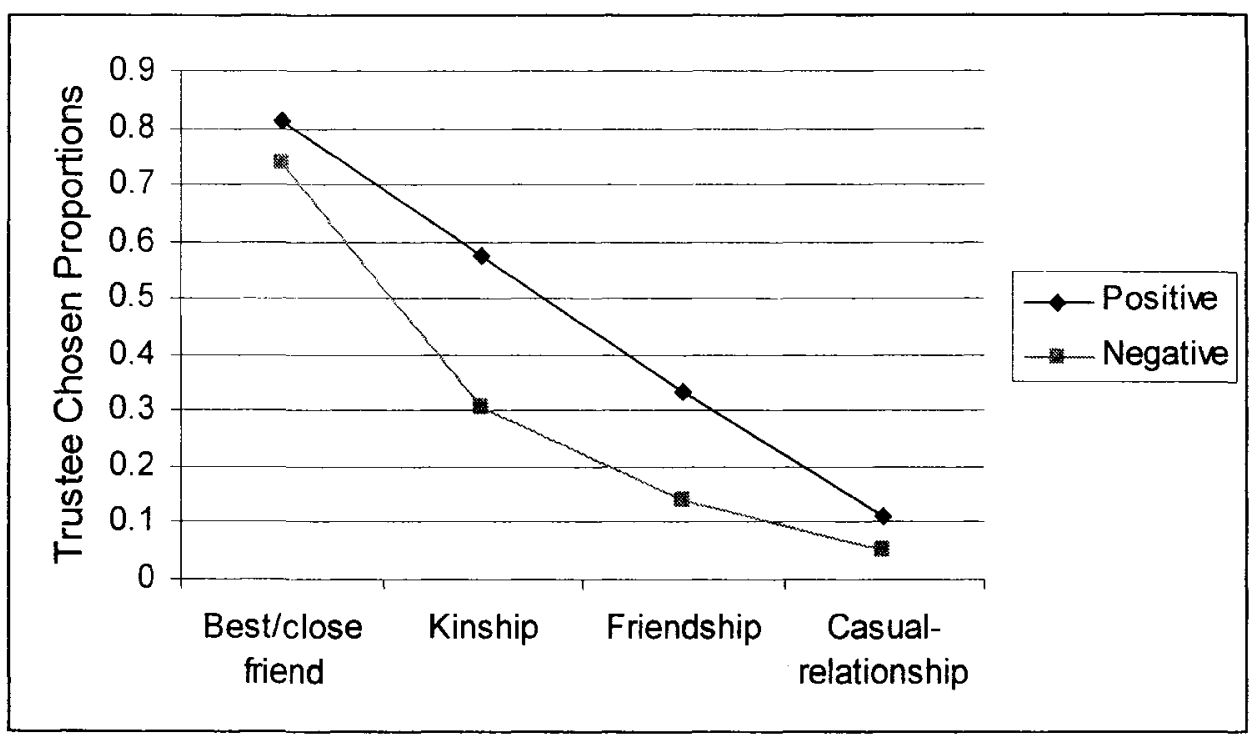

Table 6: Pair Trustee Proportion Differences of the 4 Trustee Categories within Positive and Negative as well as between Positive and Negative Personal Information Sharing Domains

\begin{tabular}{llccccc}
\hline \multicolumn{1}{c}{ Pairs of Trustee Chosen Proportion } & $\begin{array}{c}\text { Proportion } \\
\text { Differences }\end{array}$ & $\begin{array}{c}\text { Std. } \\
\text { Deviation }\end{array}$ & t & df. & Sig. \\
\hline Within & Best-friend \& Kinship & .24 & .34 & 10.10 & 201 & .000 \\
Positive & Kinship \& Friendship & .24 & .23 & 14.66 & 201 & .000 \\
& Friendship-Casual-relationship & .23 & .24 & 13.34 & 201 & .000 \\
\hline Within & Best-friend \& Kinship & .43 & .36 & 16.85 & 201 & .000 \\
Negative & Kinship \& Friendship & .17 & .22 & 10.89 & 201 & .000 \\
& Friendship-Casual-relationship & .09 & .17 & 7.07 & 201 & .000 \\
\hline Between & Best-friend & .07 & .39 & 2.65 & 201 & .009 \\
Positive & Kinship & .27 & .23 & 16.74 & 201 & .000 \\
and & Friendship & .20 & .28 & 10.22 & 201 & .000 \\
Negative & Casual-relationship & .06 & .19 & 4.32 & 201 & .000 \\
\hline
\end{tabular}

The dominant important trust position of best/close friend, regardless of the valence of the information shared, may tell us more about trust but more research is needed to continue to explore the phenomenon. The results, revealing a unique characteristic of 
best/close friend, are worthy of our attention and further discussion, since it is contradictory of some other studies; for instance, a study by Coulson (2002) showed that family is the most trusted, newspapers are the least trusted, and others are in the middle. In addition to significant differences between each of the 4 trustee categories, each category itself also shows some differences between sharing positive and negative personal information. Generally speaking, when sharing negative personal information, people seem to trust all others less than when sharing positive personal information (Figure 8). It is riskier to share negative personal information with others than to share positive personal information with others.

As can be seen from Figure 8 and Table 6, for those being trusted most and least, best/close friend and casual relationship trustees, the trusting difference between positive and negative personal information sharing is less than those being trusted in the middle, family members and friends. For example, the $81 \%$ of participants who would trust and share their positive personal information changes to $74 \%$ of participants who would share their negative information with best/close friend, not big difference; however, the $57 \%$ of people who would like to trust and share their positive personal issues drops to only $31 \%$ of people who would like to trust and share their negative personal information with their family members.

It may suggest that the valence (positive/negative) of information sharing affects trustee choosing non-averagely. Specifically the valence of the shared information influences those trustees who are on the two sides of the trusting scope (either being trusted more or less) less than those trustees who are in the middle. It seems as long as I trust you most, regardless of the content of the information, I always like to share with 
you; similarly, once I don't trust you to share my personal information with, it does not matter whether the information is good news or a happy experience. However, a basic information valence effect on trustee exists, because all trustees are trusted less when it comes to sharing bad news, a sad story etc. than when sharing good news, a happy story, a pleasant experience.

Based on the results, I would like to emphasize the characteristic of trusteechangeability. For those trustees who are trusted comparatively unchangeably, such as best/close friend, the term "Always Trustee" may be considered to use, and for those trustees who are trusted comparatively changeably, such as family members, "Changeable Trustee" may also be considered as a term to explain this phenomenon.

As a result of the above discussion, it is found that people pick out their favorite trustees, rather than randomly choosing their trustees, when sharing personal information. Best/close friend is chosen as the top trustee when sharing both positive and negative personal information. The trust circle composition is different between positive and negative personal information sharing domains; the biggest difference between the two domains happens among family members, so-called "Changeable Trustees," and the smallest change happens for best/close friend, a so-called "Always Trustee".

\section{Cultural Effects on Trust Circle Size in the Personal Information Sharing Domain}

Looking at trust as a cultural phenomenon is the domain of the cultural approach in trust research. It is important and necessary to understand how others' trust differs from our own. It is not within the scope of this study to trace historical changes in the basis and forms of trust, but rather to use cross-cultural comparisons to examine whether 
or not identifiable differences in the dominant form of groups in a culture have an impact on the size and composition of the group to whom a person will extend trust.

Figure 9 shows that in both positive and negative personal information-sharing domains, the average chosen trustee numbers of Canadians and Chinese differ. Canadians, on average, choose $7.13(S D=3.12)$ trustees to share their positive personal information with, and Chinese only choose $4.70(S D .=2.51)$ trustees on average, the 2 means varying significantly $(F(1,201)=36.90, p<0.001)$ and Canadians trusting more trustees than do Chinese. When sharing negative personal information, Chinese only choose $2.72(S D .=1.62)$ trustees on average, which is significantly less than the average of Canadian trustees chosen at $3.85(S D .=1.92)$. Chinese and Canadians vary greatly $(F$ $(1,201)=20.44, p<0.001)$ about how many trustees they trust to share their negative personal information with, and Canadians tend to trust more and Chinese tend to trust fewer people.

The results imply that culture affects people's trust circle size; Canadians' trust circle size is larger than Chinese's trust circle size in both positive and negative personal information-sharing domains. In other words, Canadians trust more others than Chinese do when sharing all kinds of personal information.

Figure 9: Mean Trustee Chosen Numbers of Canadians and Chinese in the Positive and Negative Personal Information-Sharing Domains 


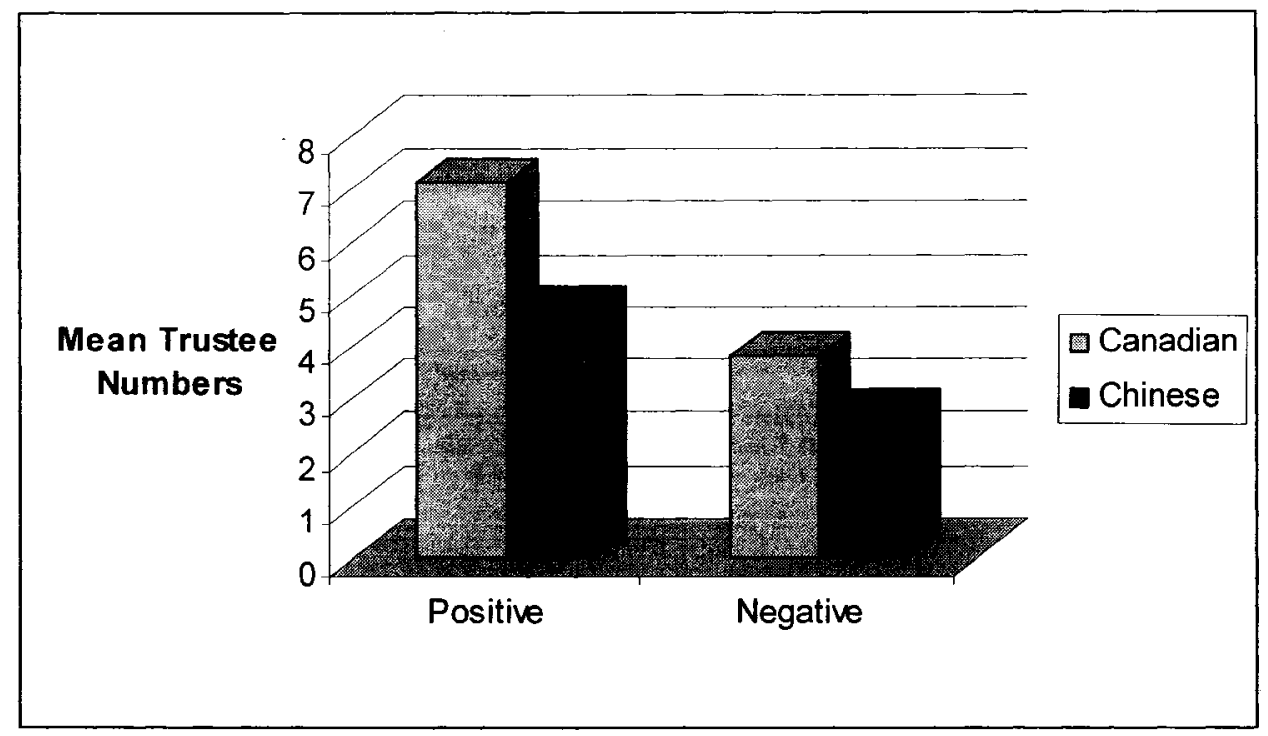

It is particularly worth pointing out that the trust circle size difference between Chinese and Canadians in the positive personal information-sharing domain is bigger than the trust circle size difference between Chinese and Canadians in the negative information-sharing domain. This means that Chinese in particular choose fewer trustees to share their positive personal information with compared with Canadians. It may confuse people to see that Chinese trust fewer others than Canadians do when not only sharing negative but also sharing positive personal information.

Chinese, as collectivists, should display their trust by telling more others about their personal information, if based on the following idea:

Collectivism as a syndrome of attitudes and behaviors embodies in (a) consideration of implications of one's own decisions and/or actions for other people, (b) sharing of material resources, (c) sharing of nonmaterial resources, (d) susceptibility to social influence, (e) self-presentation and face-work, (f) sharing of outcomes, and (g) feeling of involvement in others' lives. Individualism is, on the other hand, absence of the above (Hui \& Yee, 1994, p.410). 
According to the above, Chinese should be more likely to share their personal information, as nonmaterial resources, with others than Canadians (individualism). However, as can be seen, the data from this study do not support the general idea of what individualism /collectivism should be like. Instead, Chinese tend to hesitate in sharing their nonmaterial resources with others since Chinese trust fewer others to share their personal information with than Canadians do as the results of this study show. It may be too early to say which state is more correlated with fact at this time. Together with some other results that cannot be explained by general variables of individual-collectivism (such as Fijineman et al., 1996), it is time to begin considering whether individualism /collectivism can describe /explain the entire cultural characteristics of a nation.

While the research was conducted, some Chinese participants were asked after they finished their questionnaire why they did not trust and choose more trustees to share their good news with (got good comment, got good mark, was best student in class, university). They gave answers like: because I'm afraid people may think I'm showing off too much if I tell too many people; I won't tell many people about being best student in class because I don't want the extra attention, therefore I only tell the people who are close to me; People often don't like those who do better than you, the more positive you do compared to others, the more difficult it is to keep good relationships with others, and so on.

Unfortunately, it is very hard to find related published research to explore the phenomenon of Chinese trusting fewer others than Canadians in both positive and negative personal information sharing domains. I believe this information could shed light on the inner world of the Chinese, Canadians and their cultural differences, as well 
as correctly describing the true characteristics of the Chinese. It could be a very interesting and meaningful field of research.

\section{Cultural Effects on Trust Circle Composition in the Personal Information Sharing Domain}

As well, Figure 10 and Figure 11 show the differences between the compositions of the trust circle in the personal information-sharing domain between Canadians and Chinese separately.

In the positive personal information-sharing domain, the discriminates of trustees chosen proportions between Canadians and Chinese are all significant except for sibling (in Table 7, trustees are ranked by the amount of different $F$ between Canadians and Chinese), and Canadians trust all trustees more than Chinese do. The biggest difference for trustees chosen in the positive domain between the two groups is spouse, then psychologist/counselor, best/close friend following, and sibling showing the smallest difference in the two groups. Canadians and Chinese only trust sibling the same when sharing positive personal information, and all others trustees are trusted differently between Canadians and Chinese.

Figure 10: Proportions of Trustees Chosen by Canadians and Chinese in the Positive Personal Information-Sharing Domain 


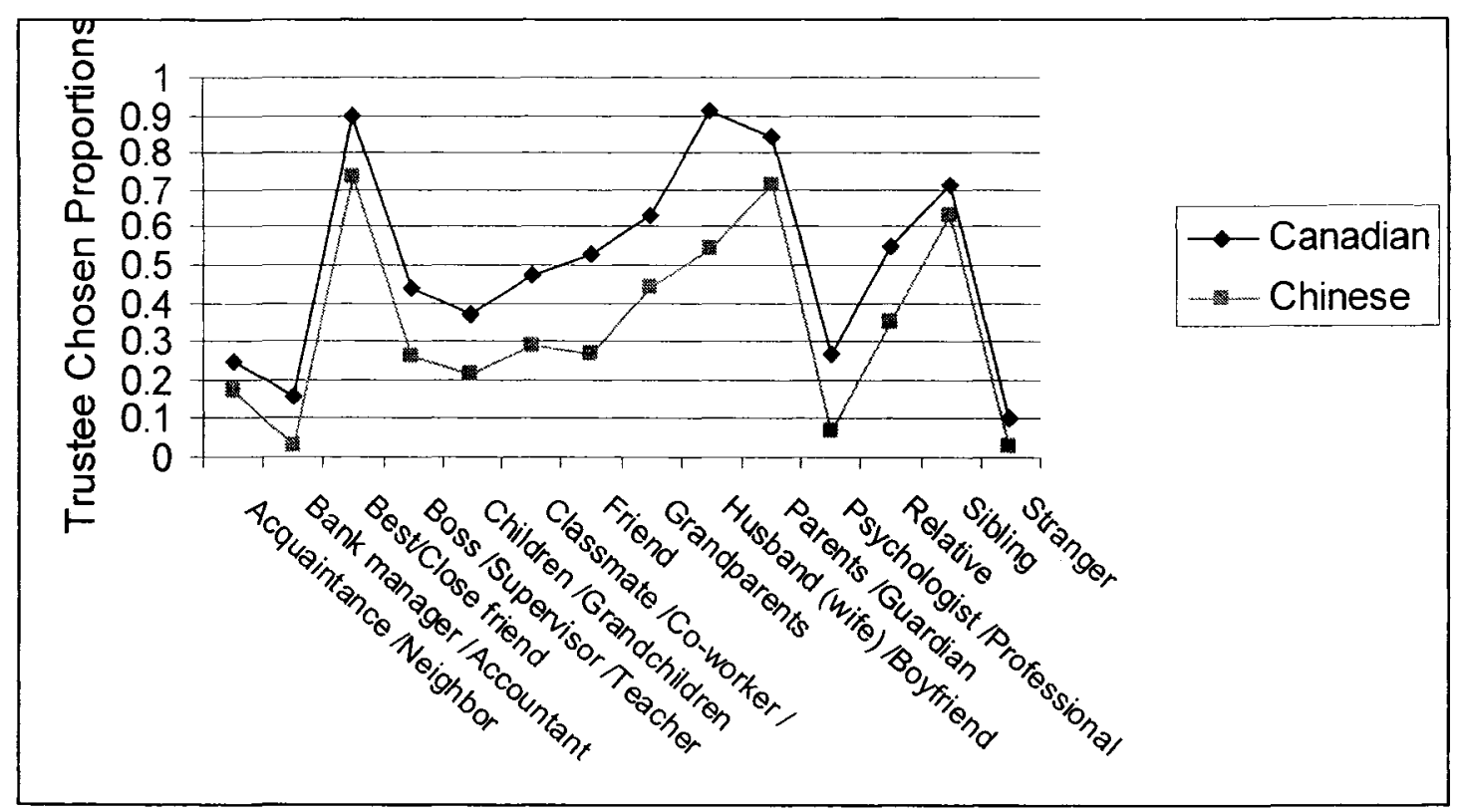

Table 7: Trustee Chosen Discriminants between Canadians and Chinese in the Positive and Negative Personal Information-Sharing Domains

\begin{tabular}{|c|c|c|c|c|c|c|c|c|c|c|}
\hline & & $\begin{array}{l}\text { Posit } \\
(\mathrm{N}=2\end{array}$ & & & & & $\begin{array}{l}\text { Neg } \\
(\mathrm{N}=\end{array}$ & & & \\
\hline & Trustee & Prop & rtion & $\mathrm{F}$ & Sig. & Trustee & Prop & rtion & $\mathrm{F}$ & Sig. \\
\hline & & Can & Chi & & & & Can & Chi & & \\
\hline 1 & Spouse /Partner & .91 & .54 & 60.05 & .000 & Parents/Guardian & .61 & .31 & 30.20 & .000 \\
\hline 2 & Friend & .53 & .27 & 26.16 & .000 & Spouse /Partner & .81 & .51 & 29.35 & .000 \\
\hline 3 & Psychologist / & .27 & .07 & 25.73 & .000 & Grandparents & .19 & .09 & 6.73 & .010 \\
\hline 4 & Best/close friend & .90 & .73 & 18.56 & .000 & Sibling & .49 & .34 & 6.45 & .012 \\
\hline 5 & Bank manager / & .16 & .03 & 16.13 & .000 & Best/Close friend & .80 & .68 & 6.37 & .012 \\
\hline 6 & Relative & .55 & .35 & 13.56 & .000 & Relative & .14 & .07 & 3.39 & .067 \\
\hline 7 & Boss/Supervisor/ & .44 & .26 & 12.66 & .000 & Children/Grand & .09 & .05 & 1.73 & .190 \\
\hline 8 & Classmate & .47 & .29 & 12.12 & .001 & Acquaintance / & .04 & .07 & 1.45 & .230 \\
\hline 9 & Grandparents & .63 & .44 & 11.79 & .001 & Boss/Supervisor/ & .11 & .08 & 1.05 & .306 \\
\hline 10 & Children /Grand & .37 & .21 & 9.72 & .002 & Stranger & .02 & .03 & 1.01 & .317 \\
\hline 11 & Parents /Guardia & .84 & .71 & 8.79 & .003 & Psychologist / & .13 & .10 & .73 & .393 \\
\hline 12 & Stranger & .10 & .03 & 6.64 & .011 & Bank manager / & .0 & .01 & .3 & .561 \\
\hline 13 & Acquaintance / & .26 & .17 & 4.63 & .033 & Friend & .23 & .21 & .21 & .647 \\
\hline 14 & Sibling & .71 & .63 & 2.45 & .119 & Classmate / & .17 & .19 & .14 & .707 \\
\hline
\end{tabular}


In the negative personal information sharing domain (Figure 11), the dominant differences of chosen proportions between Canadians and Chinese are parents/guardian and spouse with Chinese trusting and telling their parents/guardian, as well as their spouse, negative information much less than Canadians do. Regarding family members, based on the results, for those Canadians who need to share their positive personal information, they tend to first trust spouse, then parents /guardian, sibling, grandparents, relative and children/grandchildren. On the other hand, Chinese first trust parents/grandparents then sibling to share their positive personal information with, while spouse only ranks third. The ranking order of other family members for Chinese was grandparents, relative and children.

In the sharing negative personal information domain, the family member trust hierarchy of Canadians stays the same as in the sharing positive personal information domain beginning with the spouse (Figure 10, Figure 11). However, in the sharing negative information domain, Chinese shift their first 3 trustees of family members (parents /guardian, sibling, spouse/partner) to spouse/partner, sibling and parents /guardian in turn.

Similarly, as shown before, putting trust in the spouse expresses a considerable cultural difference between Canada and China. When sharing both positive and negative personal/social information, Canadians always trust their spouse more than Chinese do, since the spouse choosing rank of Canadians is always in the first rank, Chinese's spouse rank is in the fourth (positive information sharing) order and in the second (negative information sharing); the spouse choosing proportion difference between the 2 cultures is the most significant (positive, $F(1,200)=60.05, p<.000$ ) and almost the most 
significant (negative, $F(1,200)=29.35, p<.000)$. The results match those in the economic risk domain and confirm again that spouse plays a more important trust role in Western culture than in Eastern culture.

Figure 11: Proportions of Trustees Chosen by Canadians and Chinese in the Negative Personal Information-Sharing Domain

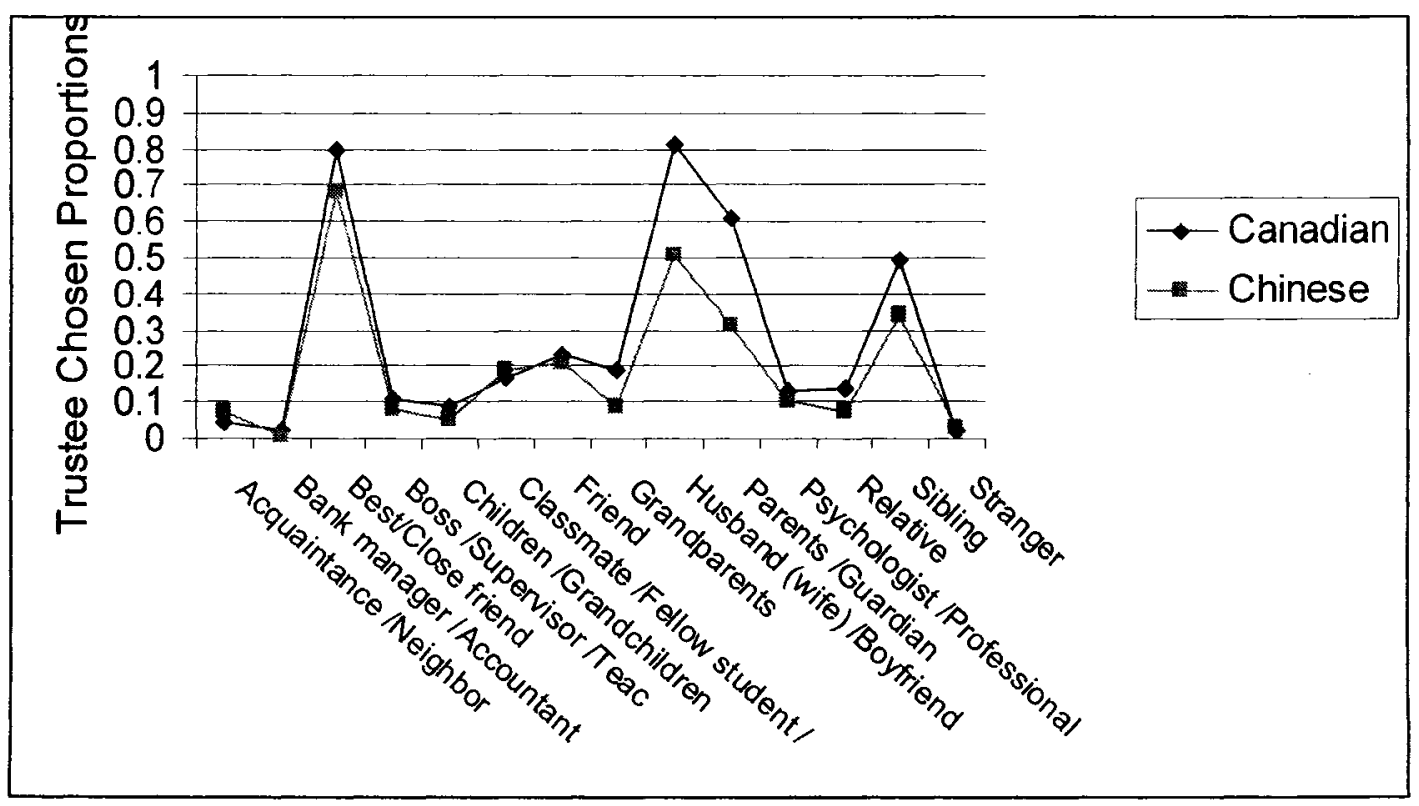

For out-of-family members, Chinese trust best/close friend more than anyone else, even more than any family members in both positive and negative personal information sharing domains; however Canadians only trust best/close friend as the second most important trustee in both positive and negative information sharing domains. These findings need to be compared with other related research in the future.

As above Figure 11 shows, Canadians still trust more for 12 of 14 trustees than Chinese when sharing negative personal information. In addition, 5 of the 14 trustees show significantly chosen discrimination between the two cultures, with the biggest 
difference being parents/guardian, the second being spouse and the third being grandparents and so on.

I further explore the 4 trustee categories (best/close friend, casual-relationship, friendship and kinship) in cultural contexts (Figure 12). When sharing good news /happy stories /pleasant experiences with others, both trust lines of Canadians and Chinese display the same slope, meaning that Canadians and Chinese trust best/close friend, family members, friends and casual-relationship with the same decreasing degree; however, Chinese show significantly less trust tendency in all 4 trustee categories (Table $8)$.

Figure 12: Four Trustee Categories Chosen Proportions of Canadians and Chinese in the Positive (left) and Negative (right) Personal Information-Sharing Domains

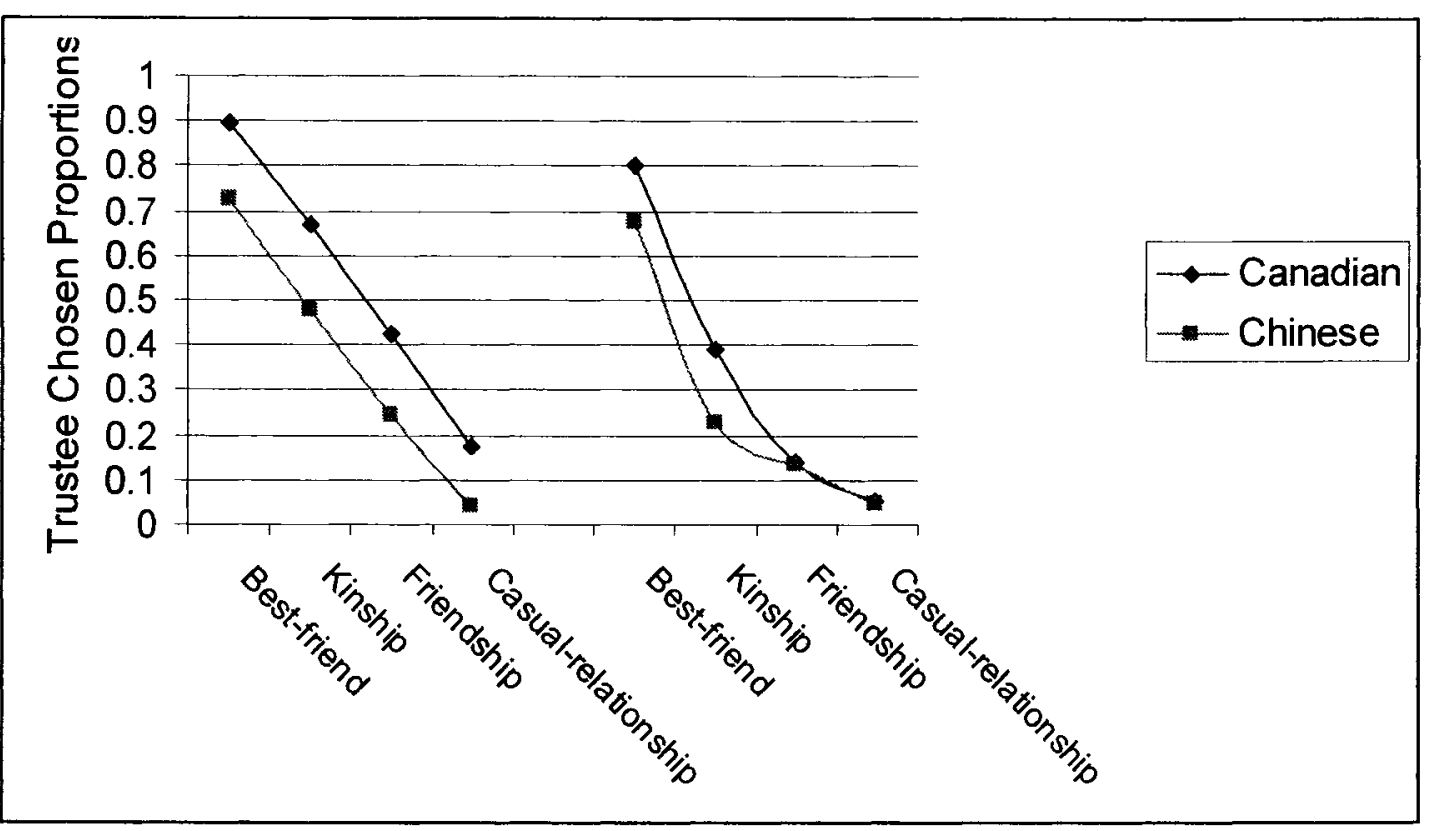

Table 8: Proportions, Standard Deviations and Differences of the 4 Trustee Categories between Canadians and Chinese in the Positive and Negative Personal Information Sharing Domains 


\begin{tabular}{lllccccc}
\hline Domain & \multicolumn{1}{c}{ Trustee } & \multicolumn{2}{c}{$\begin{array}{c}\text { Canadian } \\
(\mathrm{n}=102)\end{array}$} & \multicolumn{2}{c}{$\begin{array}{c}\text { Chinese } \\
(\mathrm{n}=100)\end{array}$} & \multirow{2}{*}{ F } & \multirow{2}{*}{ Sig. } \\
\cline { 3 - 6 } & & Prop & SD. & Prop & SD. & & \\
\hline \multirow{3}{*}{ Positive } & Casual-relationship & .19 & .26 & .04 & .08 & 30.09 & .000 \\
& Kinship & .67 & .25 & .48 & .25 & 28.69 & .000 \\
& Friendship & .43 & .31 & .25 & .26 & 20.10 & .000 \\
& Best/close friend & .90 & .20 & .73 & .35 & 18.56 & .000 \\
\hline \multirow{3}{*}{ Negative } & Kinship & .39 & .20 & .23 & .18 & 35.79 & .000 \\
& Best/close friend & .80 & .33 & .68 & .38 & 6.37 & .012 \\
& Casual-relationship & .06 & .13 & .04 & .08 & -1.99 & .159 \\
& Friendship & .14 & .18 & .14 & .17 & 0.01 & .927 \\
\hline
\end{tabular}

When sharing bad news /sad stories /unpleasant experiences, the trust differences between the 2 cultures differ. The two cultural groups show no differences when they choose general friends /classmate etc. and casual-relations to share their negative personal information with. However, choosing best/close friend and family members showed some differences between Canadians and Chinese with Chinese trusting best/close friend and family members less than Canadians in the negative personal information-sharing domain. It seems that compared with Canadians, Chinese are particularly fearful of their bad mark, bad comment (their failure), being known by their family and best/close friend, which are intimate relationships of one.

Kinship choosing represents the biggest difference between Canadians and Chinese, or Chinese are more likely to not want their family members to know they failed a course /program, they were expelled from school, they received bad comments or marks than Canadians do $(F(1,200)=35.79, p<0.001)$. It may be because Chinese tend to conceptualize family members as one whole body. For instance, Chinese parents 
usually call their children as their own flesh and blood, brotherhood is frequently seen as the relationship between hands and feet (Hwang, 1997; Hwang, 1999). If a person does a bad thing (e.g. fails school) /commits a crime etc.), then that person's parents will be held responsible for not teaching their child better. The family may suffer socially for the bad thing / crime etc. of the offspring. A long time ago, in traditional China, punishment for murder was meted out not only on the individual culprit, but also on all other family members (Bedford \& Hwang, 2003). In such traditional circumstances, in a culture of such honour/disgrace, it seems reasonable that people would not like to tell their family their bad news/sad stories when compared with people who grew up in another culture.

In summary, the cultural differences regarding trust circle composition can be described as people in each culture having their own different top trustees. Canadians and Chinese show different trustee compositions with the biggest cultural difference being spouse with Canadians trusting spouse more than Chinese do. In addition, the difference (Chinese trust fewer) when sharing positive information is bigger than when sharing negative information. I think a specific term is needed to describe the phenomenon of Chinese not only trusting fewer others when dealing with negative personal information, but also when sharing positive information. Finally, Chinese also show more hesitation when sharing their negative personal information with their family compared to Canadians.

\section{Age, Education Level, Family Income, Gender, Participants' Status and the Trust Circle in the Personal Information Sharing Domain}

Besides culture, age, gender, education, family income and participant status are 
all tested to see if they are factors influencing trust circle size and composition. It is found that they do not contribute differences to trust circle size in either positive or negative personal information sharing domains. Moreover, age, education, family income, gender and participant status all have no significant effect on trust circle composition in both the positive and negative personal information sharing domains.

\section{Conclusion about the Trust Circle in the Personal Information Sharing Domain}

Now, the questions put forward previously regarding the relationship between trust circle and the valance of the shared personal information (positive and negative), the variant of the positive/negative personal/social information, and culture can all be answered by the following results.

1. Trust circle size is decided by the valence of the personal information; positive information sharing is associated with larger trust circle size than it is when sharing negative information. Trust circle size is also affected by the variant of the valence of the shared personal information: as the variant of the positive information increases, the trust circle size tends to increase, and as the level of the negative information increases, the trust circle size tends to decrease.

2. Trust circle composition is based on the valence of the personal information shared: the hierarchy of trusting of trustees changes more in the negative information sharing domain and changes less in the positive information sharing domain; the biggest difference of chosen proportion between positive and negative information sharing is for kinship with people tending to tell their family members good news, happy stories etc. but not their bad news, sad stories etc. Best/close friend, regardless of the content of the 
shared information, tends to be trusted by most people and its hierarchy is at the top of trustees chosen.

3. Trust circle size is affected by culture; in this study, Canadians trust more other trustees than Chinese do in both positive and negative personal information sharing domains. A new, specific term may be needed to generalize the tendency of Chinese to trust fewer others not only when sharing negative personal information but also when sharing positive personal information.

Cultural issues also affect the composition of the trust circle; people in each culture have their own favored trustees in different content of shared personal information. The trust circle composition difference between the two cultures is bigger in the positive personal information sharing than in the negative; husband (wife) /boyfriend (girlfriend) /partner /lover, as spouse of family, is trusted more by Canadians than by Chinese, or spouse's trusting order is always higher for Canadians than for Chinese in both positive and negative personal information sharing. Canadians keep the same hierarchy of trust for the top 4 trustees (beginning with spouse, then best/close friend, parents /guardian and sibling) as the valence of the information changes, but Chinese choose different top trustees as the shared personal information goes from positive to negative: when sharing positive information, they begin with best/close friend, then parents/guardian, sibling and spouse; when sharing negative information, they begin with best/close friend, then spouse, sibling and parents/guardian).

4. Age, education, family income, gender and participants' status do not affect trust circle size and trust circle composition. 


\section{THE TRUST CIRCLE in DOMAIN-BASED TRUST}

The trust circle within each economic risk domain and each personal sharing domain has been discussed previously and many important findings have been indicated. However, the trust circle still needs to be explored across the two domains to see if it differs when it is in the two different domains. Thus the questions I expect to answer in the section are:

1. Whether domain affects trust circle size, 2. whether domain affects trust circle composition, and 3. whether culture influences the trust circle (size and composition). The questions will be considered in this order and the section will only deal with the circumstances in which "I trust others".

\section{Results and Discussion}

\section{Trust Circle Size in the Economic Risk and Personal Information-Sharing Domains}

Figure 13 presents the trustee chosen numbers in 4 risk variants of the economic domain, and in 4 positive and negative variants of shared personal information. I should directly compare the 2 trust circles with the 2 specific trust chosen numbers of the 2 domains, but, because the shared personal information has 2 either positive or negative valences, it is impossible to decide which trust circle size represents the trust circle size of the personal information sharing domain. Using either only the trust circle size of the positive or the negative information sharing domain or the average of the 2 trust circle sizes will not work. However, we can still see how trust circle size varies with only the 
parallel average trustee numbers chosen by participants in Figure 13 which provides a convincing picture that trust circle sizes differ depending on different domains.

Figure 13: Mean Trustee Chosen Numbers at the 4 variants of the Economic Risk Domain (left), Positive (middle) and Negative (right) Personal Information-Sharing Domains

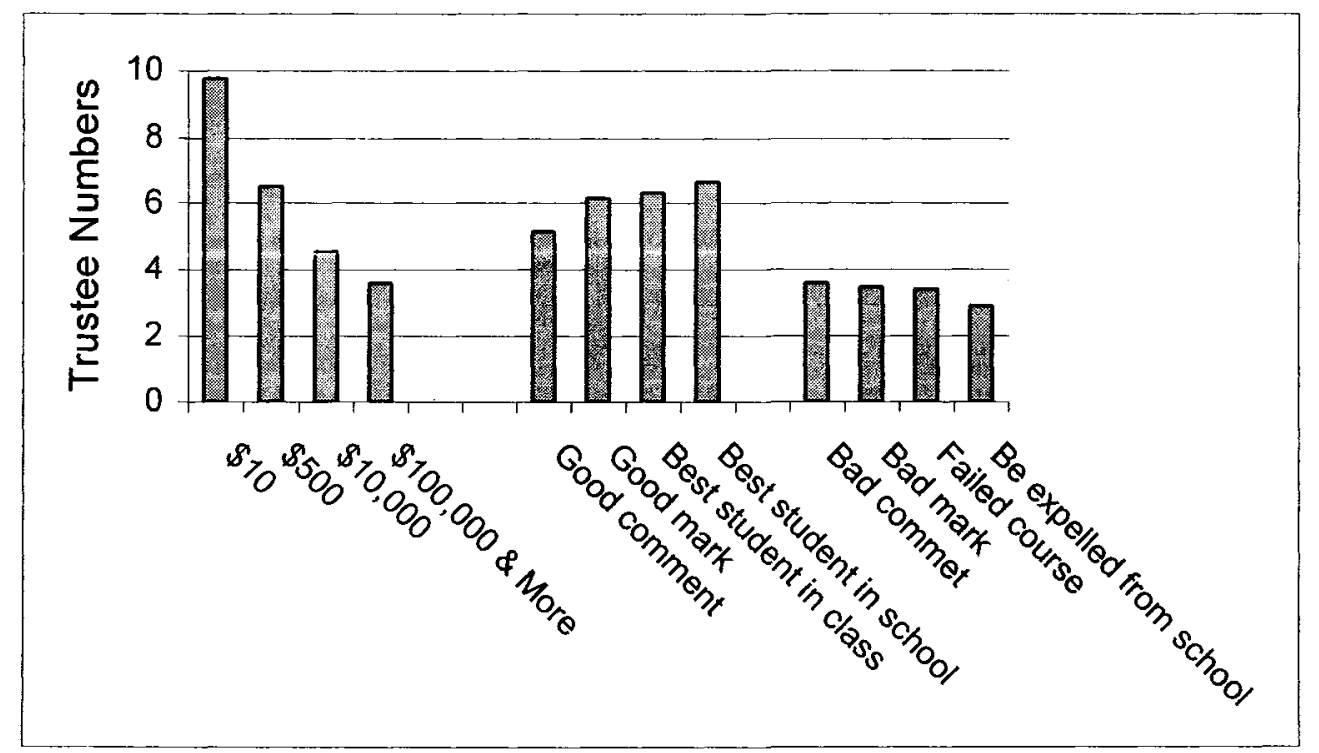

It appears that the size of the trust circle follows a different pattern in the financial domains than in the domains of sharing positive and negative personal information. People overall, in the personal domain, seem more willing to share positive than negative information. That is, in people's economic/material life, when doing things such as lending money to others, the trustee numbers tend to be different from the trustee numbers in both the positive and the negative personal information sharing domains. The findings support the general idea posted by Hardin (2001) that trust depends on context. Here we see that people adjust their trustees (add, reduce or keep the same) based on specific domains and the content of the domain. 
In addition, one can see that the number of trustees listed in the economic risk domain declines rapidly when the risk increases compared with the trust number varying in the personal information sharing context. As the variants of positive/negative information sharing domain change, the chosen trustee numbers vary, but the varying occurs gradually, not sharply. These results are further proof that the number of trustees people would like to trust is associated with domain and the valence of the circumstance.

As Figure 13 showed, in each domain, risk increases in the 4 different variants, the corresponding trustee numbers changed, further work would be necessary to ensure that in the personal domain, the information sharing examples were indeed perceived to imply different levels of risk. It would be circular to argue that issues for which there were few trustees listed, are also the riskier ones. However, the data for the economic domain, where one can be more confident about increasing levels of risk with increasing amounts lent, is consistent with the idea that trust circle size shrinks with increased risk.

Regarding the relationship between trust circle size and domain, valence and variant, the summary may help to organize: first of all, if the related domains are not the same (e.g. from economic risk to personal information sharing), the corresponding trust circle sizes differ; secondly, if the valence (e.g. positive/negative personal information) of a domain differs, the corresponding trust circle sizes tend to differ; thirdly, if the variant (e.g. economic risk level, positivity /negativity level) of the domains varies, the corresponding trust circle sizes tend to differ.

\section{Trust Circle Composition in the Economic Risk and Personal Information Sharing Domains}


Is the position of each trustee in the hierarchy the same in different domains?

Figure 14 provides us with a general picture to answer this question qualitatively. In the economic risk domains, people trust parents most, then sibling(s), followed by spouse/partner and best/close friend and so on. The trusting order of trustees in the economic domains is not the same as the trusting order of trustees in the personal information sharing domains in which best/close friend is in the most trusted position, while parents are in the second position when sharing positive information and spouse /partner is in the second position when sharing negative personal information. This may remind people of the importance of the domain which affects trust composition differently.

Figure 14: Fourteen Trustee Chosen Proportions in the Economic Risk, Positive and Negative Personal Information-Sharing Domains

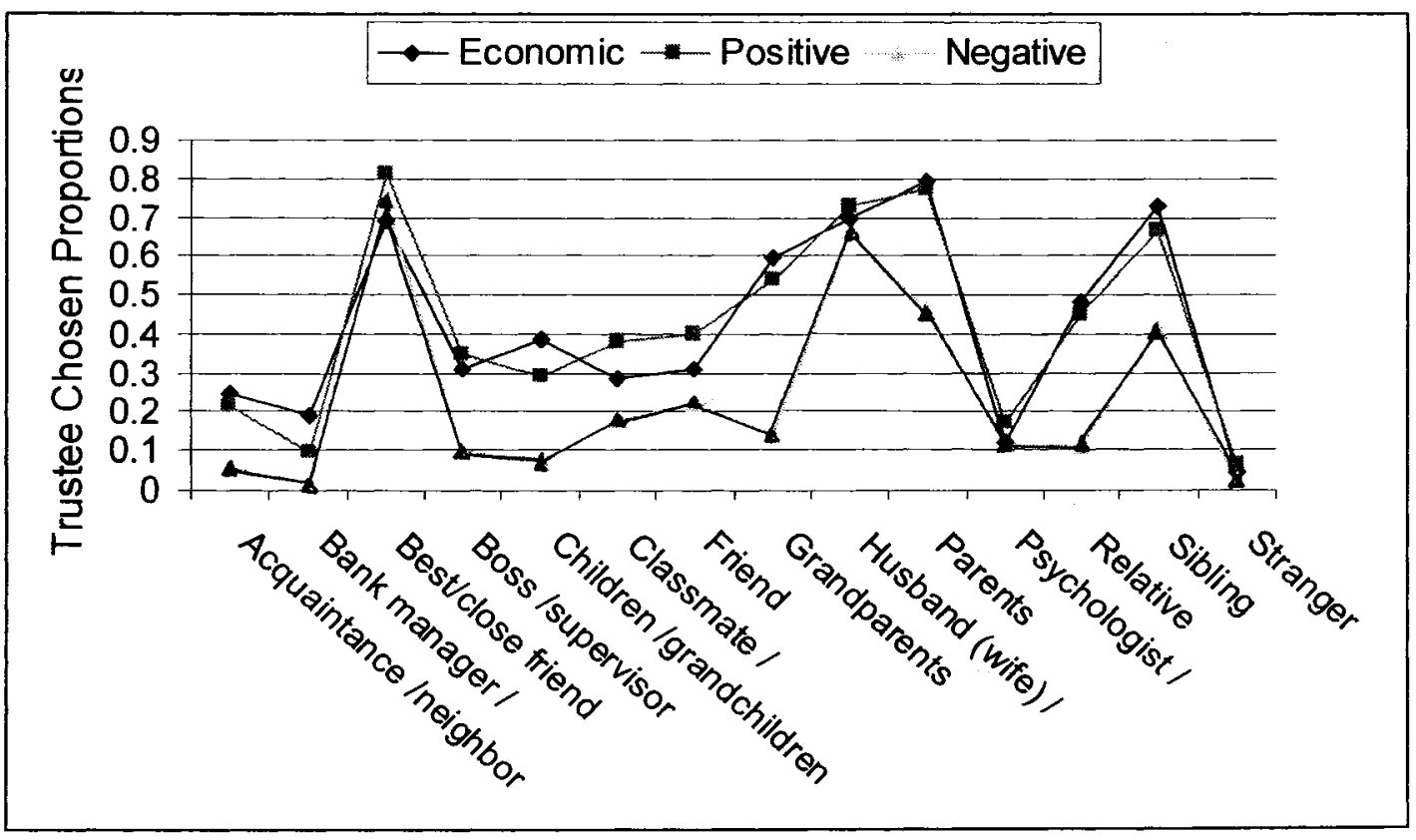


Figure 15 further shows trust circle composition in the 4 categories that are grouped from the 14 trustees as previously mentioned. On the one hand, some similarities can be seen; for example, best/close friend never falls out of the top trust position, and the casual-relationship trustee category is always trusted least in both economic risk and personal information sharing domains. On the other hand, the effect of domain on the trust circle composition can also be seen: even best/close friend is trusted dominantly in both the economic risk and the personal information-sharing domains compared with three other trustee categories, best/close friend is particularly trusted more in the personal domain than in the economic domain. Family members are trusted secondly in all 3 domains, but particularly in the economic risk domain and in the positive valence of personal information sharing; however, when sharing negative personal information, the proportion of people choosing family members drops greatly. Friends' choosing pattern is like kinship's; casual-relationship type trustees, as the least trusted trustees, tend to mirror the most trusted trustee (best/close friend) tendency.

Figure 15: Chosen Proportions of the 4 Trustee Categories in the Economic Risk, Positive and Negative Personal Information Sharing Domains 


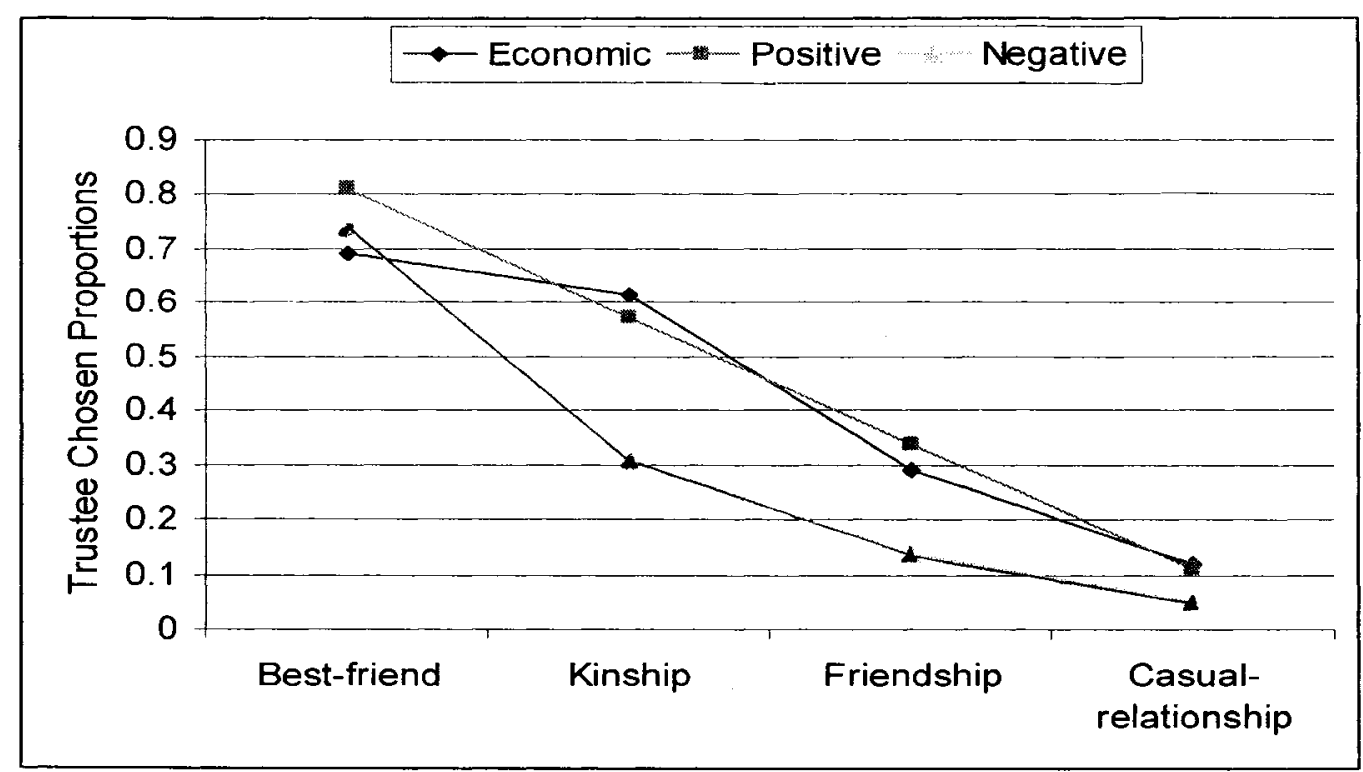

It is easy to see that the 3 lines in Figure 14 represent 3 hierarchies of trusting and corresponding relationships in the 3 different trust circumstances. If the 3 lines are not the same shape, it implies that the hierarchies of trusting and relationships represented differ; that is, the trust circle composition changes in the different domains and in the different valence of the domains. However, there is clearly a great deal of similarity in content of the trust circles across the three domains, with the majority of trustees being simply described as "close friends and family".

As mentioned, in distinct domains, in distinct valances of domains, and in distinct variants of domain or variants of valance, trust circle compositions tend to be different. For example, in the economic domain, best/close friend went from being the top trustee when lending $\$ 10$ to being only the $5^{\text {th }}$ trustee when lending $\$ 100,000$ and more; in the positive content of the personal information sharing domain, the rank of boss /supervisor /teacher went from $9^{\text {th }}$ at the lowest variant of positivity (got good comments on assignment /homework) to $7^{\text {th }}$ rank at the highest variant of positivity (best student in school /university). Also, classmate/co-worker/colleague decreased in rank from $6^{\text {th }}$ in the 
lowest negative personal information sharing domain (got bad comments on assignment /homework) to $9^{\text {th }}$ in the highest negative valence (being expelled from school /university). This, like trust circle size discussed before, also indicates qualitatively that trust circle composition is affected by the domain and the content of the domain.

In short, the composition of the trust circle is affected by the domains, or different domains (like economic risk and personal information sharing) may cause different trust circle composition. The composition of the trust circle is influenced also by the valence of the domain: if the valence of the domain is dissimilar (like positive or negative), then the trust circle composition may differ. Finally, the variant of the domain or the variant of valance of the domain may also cause the composition of the trust circle to differ if there are several variants in a domain, or in the valence of a domain.

\section{Cultural Effects on Trust Circle Size in the Economic Risk and Personal Information Sharing Domains}

Whether different cultures, such as Eastern and Western cultures, also affect the trust circle will be discussed by looking at trust circle size and trust circle composition in the two separate sections.

In Figure 16, one can see that in the same domain, participants from the two cultures included in this study, show differing mean size of the trust circle. In the economic domain, the average number of trustees chosen by Chinese $(6.48, S D=2.54)$ is larger than the trustee number of Canadians $(5.73, S D=2.03)$, and the test results revealed significant cultural effects on trust circle size $(t(2,189)=.021, p<.05)$. However, in contrast, when sharing positive personal information, the average trustee number for Canadians $(7.12, S D=3.12)$ was significantly higher than that reported by 
Chinese $(4.70, S D=2.51, t(2,192)=6.08, p<.001)$. Similarly, the average trustee number of Canadians $(3.85, S D=1.92)$ is larger than the average trustee number of Chinese $(2.72, S D=1.62)$ by a significant margin $(t(1,195)=4.52, p<.001)$ when sharing bad news /sad story /unpleasant experience etc.

Figure 16: Mean Trustee Numbers Listed by Canadians and Chinese in the Economic, Positive and Negative Personal Information-Sharing Domains

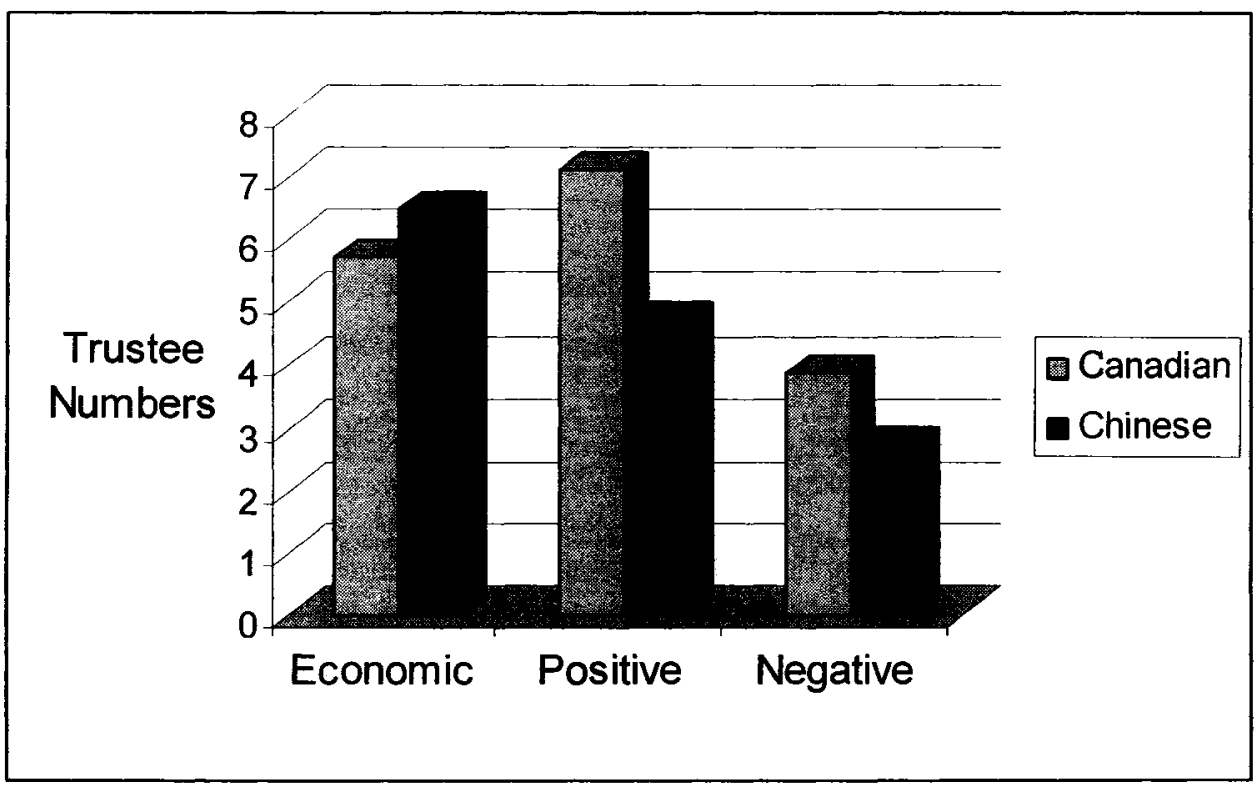

The results confirm that cultural influence on trust circle size depends on the domain of trust. Chinese respondents had a somewhat larger trust circle than Canadians for economic matters, but in the personal domain, both positive and negative valences, the Chinese listed fewer trustees than Canadians did. As long as culture differs, the trust circle size differs; all trustee circle sizes between Chinese and Canadians in all situations are significantly different.

Therefore, Chinese express their trust to more others than Canadians in the economic risk domain, but Canadians place their trust in more others than Chinese in 
personal information sharing domains, as the trust circle size of the two cultures in the 2 domains showed in Figure 16.

Further, it is found that the effects of culture on the economic risk domain are different from the effects of culture on the personal information sharing domain. That is in the former, Chinese trust circle size is larger than Canadians' trust circle size, and in the latter, the varying culture makes Chinese trust circle size smaller than Canadians'. Or in other words, Canadians trust fewer others than Chinese when facing economic domain risk, but trust more others than Chinese when thinking of whether to trust others to share their personal information with.

Canadians and Chinese place their trust in others differently in the different situations; however, the bigger trust circle size difference between Canadians and Chinese is in the positive personal information sharing domain. It implies that Chinese trust fewer others to share their good news, happy story etc. compared with Canadian do.

I would like to stress the importance of exploring the interesting phenomenon of the Chinese trusting fewer others than Canadians not only in the negative information sharing domain, but also in the positive personal information sharing domain. Further, this knowledge may reveal more about the Chinese.

Fukuyama (1995) describes the United States, Germany, and Japan as high trust cultures, and Chinese and Korea as low-trust cultures. The result s of this study contradict Fukuyama's idea. The data from the personal information sharing domain appear to support Fukuyama's idea since Chinese appear to trust fewer others (low trust culture) compared with Canadians, but the data from the economic domain do not support the idea, given that Chinese trust more others than Canadians. The contradictory results warn 
us to understand individualism-collectivism in a more profound way. I believe that

Chinese themselves are very diverse and can be described in a very wide view and that simple collectivism may not explain them in an in-depth way.

\section{Cultural Effects on Trust Circle Composition in the Economic Risk and Personal Information Sharing Domains}

Figure 17 describes how the trust circle composition of Canadians and Chinese differs in the economic risk and the personal information-sharing domains.

Figure 17: Trustee Chosen Proportions of Canadians and Chinese in the Economic Risk (left), Positive (middle), and Negative (right) Personal Information Sharing Domains

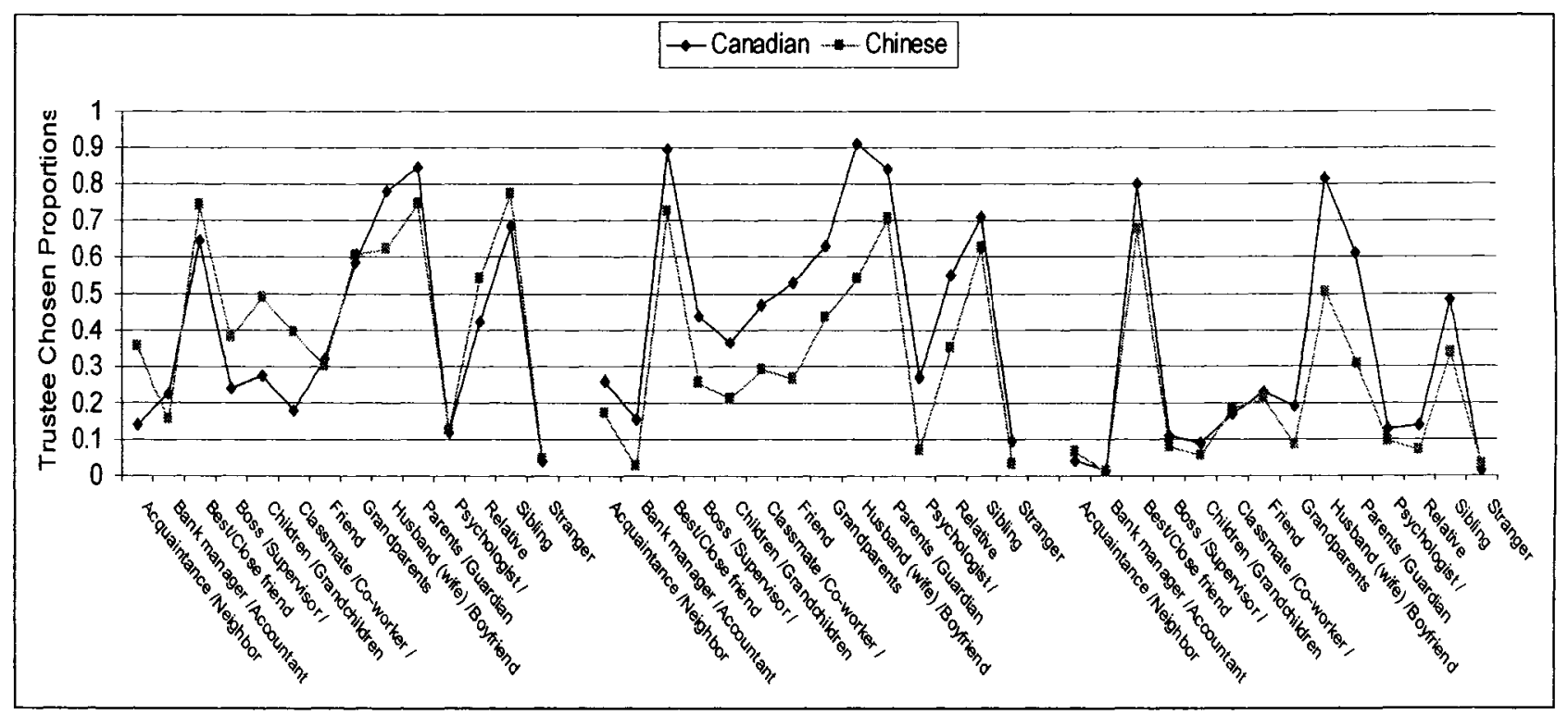

Each trusting hierarchy of each culture is described by each line of a culture in Figure 17. It is obvious that no lines of Canadians and Chinese in each specific situation overlap completely, which generally tells us that culture is a factor which affects trust circle composition. One can further see that the relationships and hierarchies of trust 
(who is trusted most, who is trusted second, third, until last) of Canadians in all 3 circumstances are different from the hierarchies of Chinese.

Generally speaking, those trustees people are more likely to trust are more important. It is meaningful to see how the top four trustees in Figure 17 are trusted by the two cultures in both the economic and personal domains. Table 9 displays the top 4 proportions of four trustees of Canadians and Chinese in the economic, as well as the positive and negative personal information sharing domains.

Table 9: The First 4 Top Trustee Chosen Proportions of Canadians and Chinese in the Economic Risk, Positive and Negative Information Sharing Domains

\begin{tabular}{llllllllllll}
\hline \multicolumn{3}{c}{ Economic } & \multicolumn{4}{c}{ Positive } & \multicolumn{3}{c}{ Negative } \\
\hline $\begin{array}{l}\text { Canadian } \\
(\mathrm{n}=102)\end{array}$ & $\begin{array}{c}\text { Chinese } \\
(\mathrm{n}=100)\end{array}$ & $\begin{array}{c}\text { Canadian } \\
(\mathrm{n}=102)\end{array}$ & $\begin{array}{c}\text { Chinese } \\
(\mathrm{n}=100)\end{array}$ & \multicolumn{2}{c}{$\begin{array}{c}\text { Canadian } \\
(\mathrm{n}=102)\end{array}$} & $\begin{array}{c}\text { Chinese } \\
(\mathrm{n}=100)\end{array}$ \\
\hline Trustee & $\mathrm{P}$ & Trustee & $\mathrm{P}$ & Trustee & $\mathrm{P}$ & Trustee & $\mathrm{P}$ & Trustee & $\mathrm{P}$ & Trustee & $\mathrm{P}$ \\
\hline Parents/ & .84 & Sibling & .77 & Spouse/ & .91 & Best-F & .73 & Spouse / & .81 & Best-F & .68 \\
Spouse / & .78 & Parents/ & .75 & Best-F & .90 & Parents/ & .71 & Best-F & .80 & Spouse/ & .51 \\
Sibling & .68 & Best-F & .74 & Parents/ & .84 & Sibling & .63 & Parents/ & .61 & Sibling & .34 \\
Best-F & .65 & Spouse/ & .62 & Sibling & .71 & Spouse/ & .54 & Sibling & .49 & Parents/ & .31 \\
\hline
\end{tabular}

In all domains and in both cultures, first of all, the first top four positions of the trust circle are occupied by the following trustees: best/close friend, spouse/partner, parents, and sibling for both Canadians and Chinese. It seems apparent that the 3 family members (parents, spouse, and sibling) and an out of family trustee (best/close friend) are the most trust-worthy trustees regardless of the culture, which points out a similarity of trustee circle composition in varying cultures. 
However, the hierarchy of the top 4 trustees of the 2 cultures in different domains and different valence of domains are not similar. Canadians rank parents/guardian first but Chinese rank them second in the economic risk domain. It is somewhat complex in the sharing of personal information: the parents' rank of Canadians is lower than the Chinese's rank in positive personal information sharing, but in the negative personal information sharing domain things are reversed with parents/guardian's ranking higher with Canadians than with Chinese. Chinese are usually more reluctant to share their negative personal information with their parents than Canadians are.

Spouse occupies a less important position in the order of trusting in Chinese culture than in Canadian culture in all circumstances; in dealing with the economic issue, Canadians rank spouse second, while Chinese rank spouse fourth. In sharing positive personal information, spouse is the most trusted by Canadians, while Chinese still only rank their spouse fourth. Even though Chinese rank spouse second in trustee ranks for sharing negative personal information, it is still lower than the first rank position chosen by Canadians. In other words, regardless of the domain, and valence of the domain, Westerners tend to trust their husband (wife) /boyfriend (girlfriend) /partner /lover more than Easterners tend to.

As for sibling's trust order, it is higher in the Chinese mind than in the Canadian mind in all different domains. The trust hierarchy of sibling of both Chinese and Canadians in the economic domain are the first and the third separately. That is Chinese are more likely to trust and lend money to their siblings than Canadians are. Faced with the sharing of positive and negative personal information, the sibling rank positions among Chinese (third) are all higher than Canadians' are $\left(4^{\text {th }}\right)$. 
Best/close friend occupies a higher trust-worthy hierarchy in Chinese's trust circle than Canadians' regardless of the domain and the content of the domain. In lending money to others, best/close friend was trusted as the third trustee and higher than spouse by Chinese, while best/close friend was trusted as the $4^{\text {th }}$ trustee, lower than spouse by Canadians. In sharing positive personal information, best/close friend is in the top trust hierarchy among Chinese, but best/close friend's trust order among Canadians is in second place. In sharing negative personal information, Chinese still treat best/close friend as the number one trustee more than any other trustees and Canadians' best/close friend is still in the second rank. This means that Chinese regard best/close friend more highly than Canadians do.

That is, even though in all domains and in both cultures, the first four trustees chosen are the same, their hierarchies are not the same. Each domain, each valence of domain and each culture has its own unique trustee order with Canadians trusting parent most and Chinese trusting sibling most in the economic domain, as well as Canadians trusting spouse most and Chinese trusting best/close friend most in both the positive and negative personal information sharing domains.

Some researchers believe that there is a sharp distinction between members of ingroups and out-groups in collectivist societies (Triandis, 1995), that Chinese cultures are highly collectivistic (Hofstede, 1980), and that the dominant in-group for Chinese has traditionally been the extended family (Huff \& Kelley, 2005). Based on this, family members (kinship category) should be chosen more often by Chinese participants, not Canadian participants; however, the facts show another story. In the economic risk domain, Chinese trust their kinship (in-group) the same as Canadians do, and in both 
positive and negative personal information sharing domains, Chinese trust their kinship members (in-group) even less than Canadians do (Figure 17).

\section{Conclusion about the Trust Circle in Domain-based Trust}

After comparing the trust circle across the 2 domains, the relationship between domain and trust is known as:

1. Trust is variable as mentioned before (Willing, 1993), and it is varied because trust circle size and composition, as 2 components of the trust circle, change. Different domains, different valences of the domain and different variants of the domain/ valence are associated with the variability of trust circle size and composition.

2. The term "Domain-based Trust" is therefore suggested to describe the changeable distinguishing features of trust.

3. Trust circle size is first affected by distinct domains, for example, the trust circle size in the economic risk domain is different from the trust circle size in the personal information sharing domain. The trust circle size is also influenced by the valence of domains if there are dissimilar valences in the domains; for instance, trust circle size in the positive personal information sharing domain is larger than that in the negative domain. Third, trust circle size is affected by the variant of the domain (different economic risk variants), when risk increases, the trust circle sizes decrease, as well as being affected by the variant of the valence (positive/negative variants of shared personal information), when positive/negative variants become stronger, the trust circle size changes.

4. Similarly, domain affects trust circle composition, for instance, in the economic domain, the trust circle composition is built with parents/guardian being in the most 
important position; and in the personal information sharing domains, the trust circle composition is built with best/close friend being in the most important rank. Second, the valence of a domain also influences the trust circle composition if the valence is applicable, for instance, family members are trusted more in the positive personal information sharing domain than in the negative information sharing domain. Thirdly, the variants of the domain /valence of the domain affect trust circle composition, for example, best/close friend's position is in the first rank when lending $\$ 10$ to others, then falls to $4^{\text {th }}$ when lending $\$ 10,000$ and finally to $5^{\text {th }}$ when lending $\$ 100,000$ and more to others.

5. Culture exerts its influence on trust circle size. When looking at both Canadians and Chinese in the economic domain, the trust circle size of Chinese is larger than Canadians' trust circle size in higher risk level, but in the personal domain, Chinese's trust circle size is greatly reduced to being smaller than Canadians' trust circle size. Chinese trust others less than Canadians do not only in sharing negative personal information, but also in sharing positive personal information.

6. Cultural issues also exert their influence on trust circle composition. For example, in the economic domain, Canadians' trust circle composition begins with parents/guardian (first) and spouse (second); Chinese's trust circle composition begins with sibling (first) and parents/guardian (second). As for the valence of domain, spouse is always trusted most by Canadians in both positive and negative personal information sharing domains, and best/close friend is always trusted most by Chinese in both positive and negative personal information sharing domains. It seems that spouse's trust role is 
more important in Western culture than it is in Eastern culture since Canadians always rank their spouse higher than Chinese do.

7. The results of the study are contradictory with some individualism /collectivism ideas, thus it is suggested that Chinese culture may not be explained wholly and thoroughly only by collectivism.

\section{TRUST OTHERS AND OTHERS TRUST ME}

Indeed, trust behavior happens in two directions, meaning that not only can Mary trust David and Tom (trust from Mary goes to David and Tom), but Mary can also receive trust from David and Tom (trust from David and Tom goes to Mary); Mary, David and Tom all play the double role of trust issuer and trust receiver.

Some researchers have considered the concept of reciprocity in their trust research (Gill, Boies, Finegan \& McNally, 2005). For example, Fijneman, Willemsen and Poortinga (1996), in their study, asked participants how much they expected to receive from and give to others. In Foddy and Dawes' (2003) trust and cooperation study, they placed two real participants in a dyad: a sender and a receiver; the sender decided how much of the endowment to send to the receiver, and the receiver also decided how much to send back to the sender. Buchan and Croson (2004) designed an experiment to explore trust boundaries, participants were randomly assigned to roles of either proposer or responder in a game which involved a proposer and a responder, and proposers and responders were all asked to state how much money they would send to the other, and how much they would expect to receive from the other. 
This dissertation clearly point out that trust happens in two ways: I trust others and others trust me and thus designed the study. In the economic domain, this affects how much money I would like to lend to others, or entrust to them, and how much I would expect to receive from others. It is important to explore trust from the two opposing directions-"I-trust-others" and "others-trust me"-, because trust happens in both directions, and it is likely that the trust relationship is reciprocal, but not entirely so. The two kinds of trust circles may not be exactly the same in the two trust loci, since their trusting directions are different; however, the two kinds of trust circles may have some things in common since they are involved in the same trust process. This section examines the trust circle in the locus of "others-trust-me", and compares how entrusting and perceived trustworthiness are related. This is done through a comparison of results about "I trust others" and "I believe others trust me" in the context of economic risk and personal information sharing domains to provide additional insight into this reciprocal trust.

In order to carry out the comparison, the questionnaires used to explore the trust circle in the locus of "others-trust-me" were parallel to the questionnaires used to examine the trust circle in the locus of "I-trust-others". Both questionnaires have the same numbers, the same sections and the same meanings; however, in order to reflect the shift from "I-trust-others" to "others-trust-me", the pronoun whom becomes who. For instance, in the economic risk domain, the question was previously "whom, do you think, you would trust to lend $\$ 10$ to, if possible?" Now the corresponding question is "Who, do you think, would trust you to lend you $\$ 10$, if possible? Choose possible person(s) from the following list, and place an X within the corresponding small box". 


\begin{tabular}{|l|l|l|l|}
\hline Acquaintance & 1 & Grandparents & 8 \\
\hline Bank manager/Accountant & 2 & $\begin{array}{c}\text { Husband (wife)/Boyfriend } \\
\text { (girlfriend)/Partner/Lover }\end{array}$ & 9 \\
\hline Best/close friend & 3 & Parents/Guardian & 10 \\
\hline Boss/Supervisor/Teacher & 4 & Psychologist/Professional counselor & 11 \\
\hline Children/Grandchildren & 5 & Relative & 12 \\
\hline Classmate/Co-worker/Colleague & 6 & Sibling & 13 \\
\hline Friend & 7 & Stranger & 14 \\
\hline
\end{tabular}

The above question is also used for three other risk levels, with only the amount of lending money varying from $\$ 500, \$ 10,000$, to $\$ 100,000$ and more separately.

In the positive personal information sharing domain, it was previously asked for the "I-trust-others" case "Whom, do you think, would you trust to share this information with, if you got good comments on your assignment/homework?" Now the participants were asked the following for the locus of "others-trust-me":

"Who, do you think, would trust you to share this information with you, if he/she had good comments on his/her assignment/homework? Choose possible person(s) from the following list, and place an X within the corresponding small box"

\begin{tabular}{|l|l|l|l|}
\hline Acquaintance & 1 & Grandparents & 8 \\
\hline Bank manager/Accountant & 2 & $\begin{array}{c}\text { Husband (wife)/Boyfriend } \\
\text { (girlfriend)/Partner/Lover }\end{array}$ & 9 \\
\hline Best/close friend & 3 & Parents/Guardian & 10 \\
\hline Boss/Supervisor/Teacher & 4 & Psychologist/Professional counselor & 11 \\
\hline Children/Grandchildren & 5 & Relative & 12 \\
\hline Classmate/Co-worker/Colleague & 6 & Sibling & 13 \\
\hline Friend & 7 & Stranger & 14 \\
\hline
\end{tabular}


The above question was also used for the three other positive levels of personal information sharing for the locus of "others-trust-me": if he/she got a good mark/grade on his/her test/examination, if he (she) was best student in his/her class, if he (she) was best student in his/her school/university.

In sharing negative personal information, the question for the locus of "I-trustothers" was phased as "whom, do you think, would you trust to share this information with, if you got bad comments on your assignment/homework?" becomes the following for the locus of "others-trust-me":

"Who, do you think, would trust you to share the information with you, if he/she got bad comments on his/her assignment/homework? Choose possible person(s) from the following list, and place an $\mathrm{X}$ within the corresponding small box".

\begin{tabular}{|l|l|l|l|}
\hline Acquaintance & 1 & Grandparents & 8 \\
\hline Bank manager/Accountant & 2 & $\begin{array}{r}\text { Husband (wife)/Boyfriend } \\
\text { (girlfriend) /Partner /Lover }\end{array}$ & 9 \\
\hline Best/close friend & 3 & Parents /Guardian . & 10 \\
\hline Boss /Supervisor/Teacher & 4 & Psychologist/Professional counselor & 11 \\
\hline Children /Grandchildren & 5 & Relative & 12 \\
\hline Classmate/Co-worker/Colleague & 6 & Sibling & 13 \\
\hline Friend & 7 & Stranger & 14 \\
\hline
\end{tabular}

The question was also used for the three other negative levels of personal information sharing for the locus of "others-trust-me": if he/she got bad marks on his/her assignment/homework, if he/she failed his/her course/program, if he (she) was expelled from his/her school /university. As noted earlier, these were intended to represent increasing levels of negative disclosure. 
Through the parallel questionnaire and comparison with previous data, we will see: 1 . whether the trust circle size of the "I-trust-others" locus is different from the trust circle size of the "others-trust-me" locus, and 2. whether trust circle composition differs across the 2 trust loci.

\section{Results and Discussion}

\section{Size of the Trust Circle: Comparing "I-trust-others" and "Others-trust-me"}

Figure 18 shows the results for trust circle size across the loci of "I-trust-others" and "others-trust-me" and how trust circle size is related to the economic risk and personal information sharing domains.

Figure 18: Mean Trustee Numbers for the Loci of "I-trust-others" and "others-trust-me" in the Economic Risk, Positive and Negative Personal Information Sharing Domains

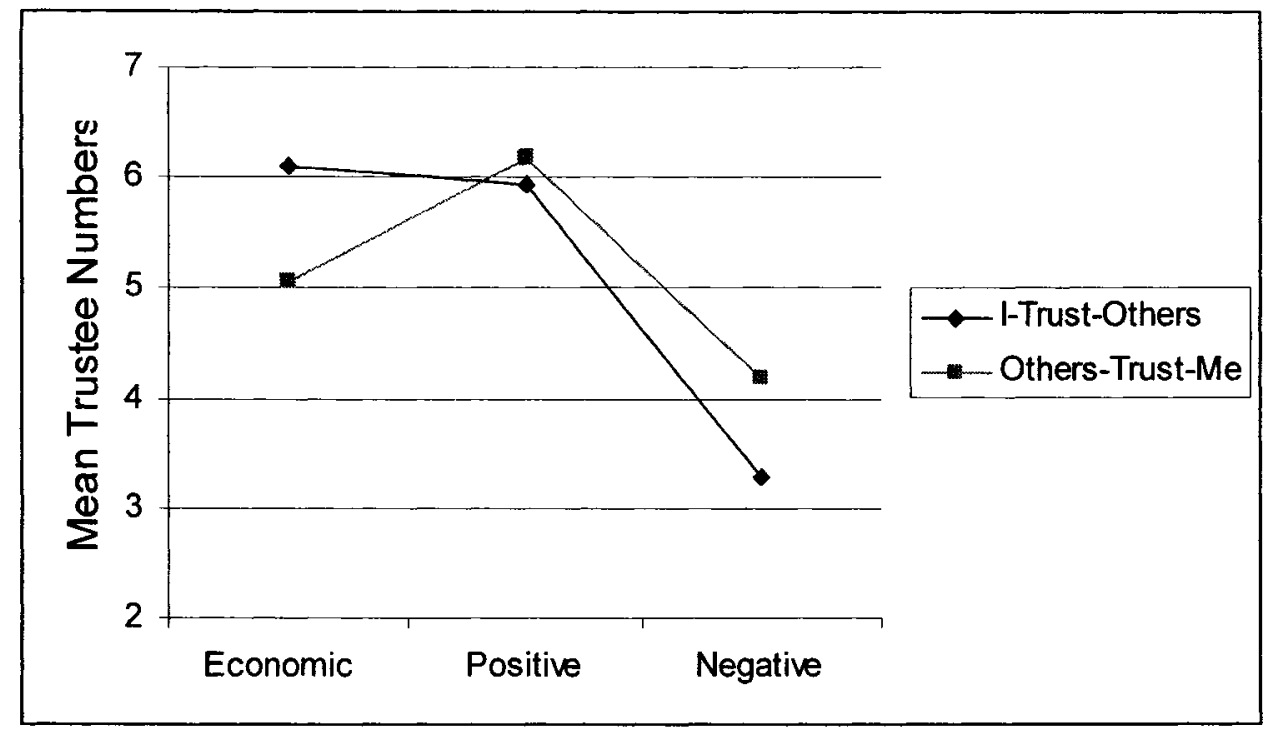

As the figure indicates, the trustee numbers chosen by participants in the locus of "I-trust-others" do not show a big difference from the mean trustee numbers in the locus 
of "others-trust-me" in the positive personal information sharing domain. That is, the trust circle size was similar for "I trust-others" $(M=5.93, S D=3.08)$ and "others trust me" $(M=6.17, S D=3.09), t(201)=1.41, p>.05$

However, there were differences across the economic risk and negative personal information sharing domains. In the economic risk domain, people believe that they would trust more others $(M=6.10, S D=2.32)$ than others would trust them $(M=5.07$, $S D=1.89$ ), showing significant differences between the 2 trust loci, entrustment vs. expectation that others will trust me $(t(201)=8.66, p<.001)$. When sharing negative personal information, people think that they would trust fewer others $(3.29, S D=1.86)$ than others would trust them $(4.17, S D=2.16)$, the difference being significant $(t(201)=$ $7.26, p<.001)$

One possible reason for these differences in trust is the fact that the risks, when lending money to others and sharing bad news /sad story /unpleasant experience with others, are larger or more serious than when sharing positive personal information. In lower risk circumstances (positive information sharing), people tend to believe others trust them the same as they trust others. However, in higher risk circumstances, people tend to believe others trust them differently from the way they trust others, for example in meeting financial risk or sharing negative issues circumstances, people believe others' trust of them is different from their trust of others, as expressed in Figure 18.

Thus one can see that the trust circle size differences between the two loci in both economic risk and the negative personal information sharing domain differ, show dissimilarities are not the same. For example, in meeting financial risk, people believe they trust others is greater than others trust them, while in sharing negative personal 
information, on the contrary, people believe others trust them is greater than they trust others.

People are generally in the belief that others will reciprocate with trust toward them. In some conditions with lower risk (such as sharing positive personal information), people's trust matches their expectation/belief about others' trust, so that the trustee numbers in both "I trust others" and "others trust me" are the same; how many others people would like to trust telling their positive information to matches how many others would trust telling other's good news to people. On the other hand, in other higher risk circumstances, say in the economic risk domain, people tend to believe fewer others would like to lend them money than they would like to lend money to. Perhaps people believe that others are not as generous as they are, in other words, people tend to believe themselves to be more openhanded/generous than others when dealing with financial leconomic issues.

Similarly, people perhaps think they are more honorable, responsible, and available than others, so more others would trust them and share others' negative personal information with them. Thus the trust circle size of "others-trust-me" is larger than the trust circle size of "I-trust-others" when sharing various bad news /sad stories /unpleasant experiences. It is expected that related researches will be done in the future to further discuss the issue.

On the basis of the results, it appears that the reciprocal nature of trust, and expected entrustment, varies across domains of trust. In the positive personal information sharing domain, comparative lower risk situation, people tended to trust others, and expected reciprocal trust from the same others. In the negative personal information 
sharing domain (higher risk situation), the trust circle size in the locus of "others-trustme" was larger than for "I-trust-others", suggesting that people usually think others would be more likely to trust them than they would be to trust others when sharing bad news, sad stories and unpleasant experiences, etc. On the other hand, the trust circle size in the locus of "I-trust-others" is larger than the trust circle size in the locus of "otherstrust-me" when meeting financial/economic risk. The reason for these distinct trust circle differences may come from the idea that people prefer to believe "I am a better person than others". As well, whether trust circle size differences emerge may be related to whether or not the risk of the domain is greater. Determining the reasons why is left for later research.

\section{Composition of the Trust Circle: Comparing "I-trust-others" and "Others-trust-} me"

In this section, the similarities and differences of trust circle composition between the two loci are discussed.

Table 10 shows how people chose the 14 potential trustees in both loci, "I-trustothers" and "others-trust-me", giving the rank order and mean proportions of people who included each of the 14 persons in their responses. Here I will focus more on the rank ordering (I made the rank order of proportions based on participant ticked each category). The most trusted others in the locus of "I-trust-others" are not always the same as those a person believes would trust them.

Table 10: Trustee Ranks and Proportions in the Loci of "I-trust-others" and "Others-trustme" in the Economic Risk, Positive and Negative Personal Information Sharing Domains 


\begin{tabular}{|c|c|c|c|c|c|c|}
\hline & \multicolumn{2}{|c|}{$\begin{array}{c}\text { Economic } \\
(\mathrm{N}=202)\end{array}$} & \multicolumn{2}{|c|}{$\begin{array}{l}\text { Positive } \\
(\mathrm{N}=202)\end{array}$} & \multicolumn{2}{|c|}{$\begin{array}{l}\text { Negative } \\
(\mathrm{N}=202)\end{array}$} \\
\hline & $\begin{array}{l}\text { I trust } \\
\text { others }\end{array}$ & $\begin{array}{l}\text { Others } \\
\text { trust me }\end{array}$ & $\begin{array}{l}\text { I trust } \\
\text { others }\end{array}$ & $\begin{array}{l}\text { Others } \\
\text { trust me }\end{array}$ & $\begin{array}{l}\text { I trust } \\
\text { others }\end{array}$ & $\begin{array}{l}\text { Others } \\
\text { trust me }\end{array}$ \\
\hline & $\begin{array}{c}\text { Rank } \\
\text { (Prop) }\end{array}$ & $\begin{array}{c}\text { Rank } \\
\text { (Prop) }\end{array}$ & $\begin{array}{c}\text { Rank } \\
\text { (Prop) }\end{array}$ & $\begin{array}{c}\text { Rank } \\
\text { (Prop) }\end{array}$ & $\begin{array}{c}\text { Rank } \\
\text { (Prop) }\end{array}$ & $\begin{array}{c}\text { Rank } \\
\text { (Prop) }\end{array}$ \\
\hline \multirow[t]{2}{*}{ Parents /Guardian } & 1 & 1 & 2 & 4 & 3 & 4 \\
\hline & $(0.80)$ & $(0.83)$ & $(0.77)$ & $(0.59)$ & $(0.46)$ & $(0.43)$ \\
\hline \multirow[t]{2}{*}{ Sibling } & 2 & 3 & 4 & 3 & 4 & 3 \\
\hline & $(0.73)$ & $(0.67)$ & $(0.67)$ & $(0.75)$ & $(0.41)$ & $(0.62)$ \\
\hline \multirow{2}{*}{$\begin{array}{l}\text { Husband (wife)/Boyfriend } \\
\text { (girlfriend) /Partner /Lover }\end{array}$} & 3 & 2 & 3 & 2 & 2 & 2 \\
\hline & $(0.70)$ & $(0.73)$ & $(0.73)$ & $(0.80)$ & $(0.66)$ & $(0.73)$ \\
\hline \multirow[t]{2}{*}{ Best/Close friend } & 4 & 4 & 1 & 1 & 1 & 1 \\
\hline & $(0.70)$ & $(0.65)$ & $(0.81)$ & $(0.88)$ & $(0.74)$ & $(0.81)$ \\
\hline \multirow[t]{2}{*}{ Grandparents } & 5 & 5 & 5 & 9 & 7 & 9 \\
\hline & $(0.60)$ & $(0.52)$ & $(0.54)$ & $(0.40)$ & $(0.14)$ & $(0.21)$ \\
\hline \multirow[t]{2}{*}{ Relative } & 6 & 6 & 6 & 7 & 9 & 7 \\
\hline & $(0.48)$ & $(0.39)$ & $(0.45)$ & $(0.49)$ & $(0.11)$ & $(0.25)$ \\
\hline \multirow[t]{2}{*}{ Children /Grandchildren } & 7 & 7 & 10 & 8 & 11 & 8 \\
\hline & $(0.38)$ & $(0.25)$ & $(0.29)$ & $(0.42)$ & $(0.07)$ & $(0.22)$ \\
\hline \multirow[t]{2}{*}{ Boss /Supervisor /Teacher } & 8 & 10 & 9 & 11 & 10 & 11 \\
\hline & $(0.31)$ & $(0.20)$ & $(0.35)$ & $(0.25)$ & $(0.10)$ & $(0.08)$ \\
\hline \multirow[t]{2}{*}{ Friend } & 9 & 8 & 7 & 5 & 5 & 5 \\
\hline & $(0.31)$ & $(0.24)$ & $(0.40)$ & $(0.54)$ & $(0.22)$ & $(0.35)$ \\
\hline Classmate /Co-worker & 10 & 9 & 8 & 6 & 6 & 6 \\
\hline /Colleague & $(0.29)$ & $(0.21)$ & $(0.38)$ & $(0.50)$ & $(0.18)$ & $(0.28)$ \\
\hline \multirow[t]{2}{*}{ Acquaintance } & 11 & 11 & 11 & 10 & 12 & 10 \\
\hline & $(0.25)$ & $(0.17)$ & $(0.22)$ & $(0.28)$ & $(0.06)$ & $(0.10)$ \\
\hline \multirow[t]{2}{*}{ Bank manager/Accountant } & 12 & 12 & 13 & 13 & 14 & 14 \\
\hline & $(0.19)$ & $(0.15)$ & $(0.09)$ & $(0.09)$ & $(0.01)$ & $(0.03)$ \\
\hline \multirow{2}{*}{$\begin{array}{l}\text { Psychologist /Professional } \\
\text { counselor }\end{array}$} & 13 & 13 & 12 & 12 & 8 & 13 \\
\hline & $(0.11)$ & $(0.05)$ & $(0.17)$ & $(0.10)$ & $(0.11)$ & $(0.03)$ \\
\hline \multirow[t]{2}{*}{ Stranger } & 14 & 14 & 14 & 14 & 13 & 12 \\
\hline & $(0.01)$ & $(0.01)$ & $(0.06)$ & $(0.09)$ & $(0.03)$ & $(0.05)$ \\
\hline
\end{tabular}

The trusting hierarchies of the loci of "I-trust-others" and "others-trust-me" varied, although overall, they are remarkably similar. The largest difference occurred in 
the positive personal information sharing domain: when the trust locus changes from "Itrust-others" to "others-trust-me", 6 out of 11 trustee ranks go up while 5 trustee ranks go down. The smallest composition variance happens in the economic risk domain: 2 out of 5 trustee ranks go up and 3 go down when the trust locus changes. The composition change in the negative information sharing circumstance is in the middle with 5 out of 9 trustees ( 3 ranks go up, and 2 ranks go down) changing their ranks. That is, the priority of trust in the locus of "others-trust-me" differs from the priority of trust in the locus of "Itrust-others".

The phenomenon may reveal an important finding. When the domain involves economic risk and the trust locus becomes "others-trust-me", the choice of spouse, friend, and classmate/co-worker/colleague increases in frequency; that is people think that their spouse, friend, or classmate would trust them more than they would trust these trustees. At the same time, the ranks of sibling and boss /supervisor /teacher go down, meaning that people believe their sibling and boss /supervisor/teacher would trust them less than they would trust these trustees. But again, the reciprocity between "I trust person x" and "Person trusts me $\mathrm{x}$ " is strong and is an important result.

In the personal information sharing domains, a comparison between trust in "Iothers-others" and "others-trust-me" shows more differences. First, the ranks of the 7 trustees (sibling, spouse, children/grandchildren, friend, classmate/co-worker/colleague, acquaintance /neighbor, stranger) are higher in both the positive and negative examples. . Second, expected trust from 4 trustees (parents, grandparents, boss /supervisor /teacher, psychologist /counselor) is lower in both positive and negative personal information sharing domains. Finally, relative (a family member) expresses a contradictory tendency 
with the rank going down in the positive domain but going up in the negative domain when the trust locus of "I-trust-others" changes to that of "others-trust-me".

The ranks varying of "I-trust-others" and "others-trust-me" suggests that people think that older generations, higher authorities etc. such as parents /guardian, grandparents, boss /supervisor /teacher, and psychologist would trust them less than they would trust those trustees or people believe that they receive those trustees' trust less than they give their trust to those trustees, since people rank the older generations, higher authorities etc. lower in the locus of "others-trust-me" than in the locus of "I-trustothers". On the contrary, people tend to believe that those of the same generation, younger generations, subordinates etc. such as spouse /partner, sibling, children /grandchildren, friend, classmate /co-worker/colleague, acquaintance and stranger tend to trust them more than they would trust those trustees based on the fact that people rank the same and younger generations, subordinates etc. higher in the locus of "others-trust-me" than in the locus of "I-trust-others".

This was not a focus of the study as originally designed, but is an interesting finding and reflects a consensual understanding of who, in a given culture, is trustworthy. Among all 14 trustees, only best/close friend and bank manager/accountant were chosen equally often in both loci of "I-trust-others" and "others-trust-me". That is, people tended to believe that they would trust their best/close friend the same as their best/close friend trusts them. It may demonstrate again the unique place of best/close friend in trust; unlike other trustees who are either trusted more or less by people when the trust locus changes, best/close friend always retains an equal position regardless of the trust locus. People have an equal relationship with best/close friend when trust is involved. When it 
comes to bank manager /accountant, the casual relationship trustee is also trusted the same in the two trust loci.

It is noteworthy to point out several characteristics of the best/close friend and bank manager /accountant relationships which possess the same trust priority in both trust loci: first of all, they are non-family members; secondly, they are chosen based on people's own will and desire, rather than some other reasons, indeed people cannot choose their own teacher, supervisor, and co-worker etc., but people can choose their best/close friend and their bank manager /accountant; thirdly, they are the representatives of 2 typical relationships in trust, one being the most intimate personal relationship and the other being a pure occasional business relationship. In addition, the unique position of best/close friend is confirmed as expressed previously in the trust circle in the locus of "Itrust-others".

\section{Conclusions about the Trust Circle in the Two Trust Loci}

The parallel questions about the two trust loci allow a comparison with respect to the other central variables of this study. Through the parallel questionnaire items, and comparison with previous data, we will find out: 1 . whether the trust circle size of the "Itrust-others" locus is different from the trust circle size of the "others-trust-me" locus, and 2. whether trust circle composition differs across the 2 trust loci.

The concept of the trust locus demonstrates 2 trust forward directions in "I trust others" and "others trust me" and the 2 trust loci are meaningful and important to further trust research.

The results of this section demonstrate the following: 
Trust circle size and trust circle composition in different trust loci differ, trust in the "I-trust-others" locus seems to be different from trust in the "others-trust-me" locus. That is, people usually believe that how they trust others tends to not be equal to how others trust them.

Trust locus is a factor affecting trust circle size. The trust circle size in the locus of "I-trust-others" is larger than the trust circle size in the locus of "others-trust-me" in the economic risk domain, meaning people believe they would give trust to more others than they would receive from others when meeting a financial/economic risk. The result implies that people may think they are more generous and helpful than others.

When the shared personal information is positive, the trust circle size in the locus of "I-trust-others" and the locus of "others-trust-me" does not show much difference; that is in a comparatively lower risk domain, how many others people trust to share a positive issue with is almost equal to how many others trust people to share others' happy story /good news etc with. But when the shared personal information is negative, in a comparatively higher risk domain, trust circle sizes in the two trust loci are significantly dissimilar, trust circle size in the locus of "others-trust-me" being larger than trust circle size in the locus of "I-trust-others". It is suggested that people tend to believe others would trust them sharing others' negative issues more than people trust others with people's negative personal information; thinking that they are a better person may be hidden in the results.

The trust circle compositions in the loci of "I-trust-others" and "others-trust-me" also vary either by going up or down the corresponding ranks from one trust locus to another. A striking example is that people believe that those of older generations, 
authorities, respected professionals, such as parents/guardian, grandparents, boss /supervisor/teacher, and psychologist tend to be trusted by people more than they trust people. On the contrary, those who have an equal or subordinate relationship with people, such as spouse, friend, classmate /co-worker /colleague etc. tend to be trusted more in the locus of "others-trust-me" than in the locus of "I-trust-others", revealing that people think that their spouse, friend, classmate/co-worker/colleague, acquaintance/neighbor, and children/grandchildren would trust them more than they would trust these trustees. Another interesting finding is that only 2 non-family members, best/close friend and bank manager/accountant are trusted equally between people trust them and they trust people.

\section{GENERAL CONCLUSION}

This study has made an original contribution to trust research, particularly through the development of some new concepts: trust circle, domain-based trust, and locus of trust. The results also demonstrate the importance of cultural background as a factor in trust.

\section{Concept}

The trust circle includes 2 components, trust circle size, which reflects the number of people being trusted, and trust circle composition, which reflects who is being trusted, and hierarchy of trusting. The 2 components together build a fuller trust circle picture.

Domain-based trust expresses the point that trust varies: it changes across different domains (such as the economic domain and the personal social information sharing domain); it changes with the different valences of the domain (positive and 
negative shared personal/social information), and also based on the different variants given in the domain (lending $\$ 10, \$ 500, \$ 10,000$ etc ) and/or given in the valence of domain (such as. getting a good/bad comment, getting a good/bad mark etc.).

The concept of locus of trust, not previously examined, shows that there is merit in examining 2 loci of trust "I-trust-others" and "others-trust-me", which indicates twoway trust forward directions with both trust loci together providing a more complete trust circle description. Locus of trust was used in the study to establish whether trusting others is the same or different from expecting others to trust us. There were some differences in trusting others, and beliefs others would trust self.

Culture plays an important role in trust by influencing trust circle size and trust circle composition; different cultures have their own corresponding unique trust circle.

\section{Trust Circle Size}

This study's results suggest that the trust circle size in the economic risk domain is larger than the trust circle size in the personal/social information sharing domain, meaning people tend to trust more others in meeting economic risk than in dealing with personal information issues. Trust circle size in the positive personal information sharing domain is larger than that in the negative personal information sharing domain, meaning people tended to show more prudence when the information they might share with others was of a negative nature, such as bad news, sad story, and unpleasant experience. In addition, as the economic risk variant increases, people tend to trust others less; similarly when the shared personal information becomes more negative, people are inclined to trust fewer others. However, in the positive personal information sharing domain, the stronger the variant of positivity, the more others people will trust to share with. 
The trust circle size for the Canadian sample, in the higher economic risk domain, is smaller than the Chinese trust circle size, suggesting that Chinese respondents tend to trust more others than Canadians when higher economic risk was involved. However, Chinese respondents' trust circle size in the personal/social information sharing domain is smaller than that reported by the Canadian sample in both positive and negative information sharing. The finding that Chinese reported that they trust fewer others compared with Canadians not only in negative but also in positive personal information sharing domains is worthy of mention. This may reflect the fact that personal reputation has more extensive consequences in Chinese culture than in Canadian. This speculation presents a challenge for future research. Nevertheless, it underlines the very high importance of the concept of personal relationships in China as discussed in the introduction

People with a higher level of education are more likely to trust more others than those who have a lower education level in the economic risk domain.

\section{Trust Circle Composition}

This study's results suggest that trust circle composition changes across different domains; it shows that people may have an understanding of the consequences of trusting others with personal information, particularly negative personal information, as opposed to trusting someone to lend money to. For example, in the study, a parent /guardian was trusted most in the economic risk domain, while best/close friend was most frequently nominated as the most trusted in the personal/social information sharing domain. Secondly, trust circle composition is also influenced by the different valence (positive and negative) of domains. For instance, in the positive personal social information 
sharing domain, people were inclined to trust family members more than they trusted outof-family members, and for negative valence examples, people trusted family members most. Third, as the variant of the domain varies, the corresponding trust circle compositions also differ; for example, the trusting order of best/close friend becomes lower as the risk variant of the economic domain increases. The data also suggest that there is some consistency in the composition of the trust circle across variants of positive personal information sharing domains.

An important finding of the differences between Canadians and Chinese in trust circle composition is that Canadians always put spouse in a higher trusting order than do Chinese respondents in all circumstances. In addition, Chinese are more likely than Canadians to change their top trustees across domains. This may indicate a higher sensitivity to relationships and the importance of reputation (Yuki, et al., 2005).

\section{Loci of the Trust Circle}

The results of this study confirm that the trust circle in the locus of "I-trustothers" tend to be different from the trust circle in the locus of "others-trust-me" in some situations; that is people believe that how they trust others is not always equal how others trust them.

Specifically, the trust circle size in the locus of "I-trust-others" is larger than the trust circle size in the locus of "others-trust-me" when people are involved in the economic/financial risk domain, showing that people believe they trust more others than others trust them. On the contrary, trust circle size in the locus of "I-trust-others" is smaller than the trust circle size in the locus of "others-trust-me" when the personal /social information shared is negative, displaying that people believe others would like to 
trust them with others' bad news, sad stories etc. more than they trust others to share such negative information with. When the personal/information shared is positive, like happy story, good news, pleasant experience etc., the trust circle sizes in both loci of "I-trustothers" and "others-trust-me" do not show big differences.

In addition, several interesting features of trust circle composition between the loci of "I-trust-others" and "others-trust-me" are worthy of mention. People believe that those from older generations, superiors, and those of high social standing, such as parents /guardian, grandparents, boss /supervisor /teacher and psychologist tend to trust them less than they trust those particular trustees. Contrasting this, those of equal or younger generations or with equal relationships, such as spouse, children /grandchildren, friend, classmate /co-worker /colleague, and acquaintance /neighbor are inclined to trust them more than they trust those particular trustees. As non-family members, best/close friend (intimate relation for focal person) and bank manager/accountant (pure occasional relationship) tend to trust people to the same degree that people trust them.

Some results of the study, particularly about the Chinese, seem to contradict the ideas of individualism /collectivism and this may suggest that the complexity of a culture (such as China) may not be explained completely only by individualism/collectivism; individualism /collectivism may coexist.

\section{LIMITATIONS and FUTURE RESEARCH}

Because this study was an exploratory one, there are several things which could be improved in future research. 
It is challenging to design different meaningful variants in the different domains or valence of domains. The four variants (lending $\$ 10$ to others, lending $\$ 500$ to others, lending $\$ 10,000$ to others etc.) in the economic risk domain seemed to be effective in this study since, in general, a set of numbers is comparatively easy to divide into suitable and numerous levels. On the basis of the differences in size of trust circles for the different amounts, the participants seemed to differentiate among the levels. However, in the sharing personal information domain, the four variants of positive and negative personal information (such as "got bad comments", "got a bad mark", "failed course", etc.) perhaps did not provide enough difference between each variant, so the differences of the trust circle size between each variant does not show a big difference. This may reflect the fact that people do not make distinctions in the personal domain in the way they do in the financial domain. However, it is acknowledged that the results may be specific to the particular examples. Future research should develop a larger base of examples, and incorporate situations such as intimate relationships (e.g. asking about romantic partners). The literature on self-disclosure may prove some suggestions for such development.

In addition, it is unfortunate that it may not be possible to create questions which would operationalize levels of risk, and valence of risk, across the economic and personal domains. The study was initially planned with the idea of increasing levels of risk in all three domains, but the data do not clearly demonstrate this, and the economic and personal domains may not be comparable in this sense. Future research will be needed to establish that this is indeed the case.

Another possible concern may be raised about the sample of cultures. Only two cultures have been sampled, so one may be cautious in drawing conclusions about 
possible universals. However, there were sound reasons for making the CanadianChinese comparison, on the basis of a research literature pointing to trust relevant differences in social structure and family relationships.

During the study, it would be better if both Chinese and Canadian participants could participate in the same environment, even though research conditions in the two countries differ; for example, university students in Canada answered the questions using a computer while university students in China answered the questions by pen. Also, the general subsample people in Canada participated in the study individually, whereas the general sample of people in China participated in groups. Perhaps this difference in testing environment had the effect of emphasizing cultural differences, but it is not possible to tell if this was the case.

Another potential shortcoming of the study is the lack of quantitative analysis of the rank order of chosen trustees. Throughout the study, I realize that using ranks to analyze the composition of the trust circle would be more meaningful and logical, especially when comparing any two compositions. However, it is very difficult to find a statistical method which can analyze rank and that is why I did not have related quantitative ranking analysis, because the respondents in the study did not provide rankings themselves; the ranks were simply based on the size of the proportion of individuals who ticked a particular trustee. As noted earlier, because of the differences across the domains, it was not considered reasonable to test for the similarity in ranks across the three domains.

Finally, more diversified domains as well as valences and variants of the trust circle are needed to confirm the related conclusions of the study. In addition, designing 
more studies to further explore how I trust others and how others trust me is another interesting challenge for future research, with the potential to greatly extend our field of vision.

The most important part of the study is to point to future work which might flow from it and to increase understanding of the extent of current trust knowledge. The study also produces some suggestions in the new research direction. 


\section{Reference}

Akerlof, G. (1997). Social distance and social decision. Econometrica, 65, 1005-1027.

Atuahene-Gima, K., \& Li, H. (2002). When does trust matter? Antecedents and contingent effects of supervisee trust on performance in selling new products in China and the United Stated. Journal of Maketing, 66, 61-81.

Bedford, O. \& Hwang, K. K. (2003). Guilt and shame in Chinese culture: A crosscultural framework from the perspective of morality and identity. Journal for the Theory of Social Behavior, 33, 127-144.

Berg, J. Dickhaut, J. \& McCabe, K. (1995). Trust, reciprocity, and social history. Games and Economic Behavior, 10, 122-142.

Berry, J. W. (1974). Canadian psychology: Some social and applied emphases. Canadian Psychologist, 15, 132-139.

Berry, J. W. (2000). Cross-cultural psychology: A symbiosis of cultural and comparative approaches. Asian Journal of Social Psychology, 3, 197-205.

Berry, J. W., Poortinga, Y. H., Segall, M. H. \& Dasen, P. R. (1992). Social Behavior. In J. W. Berry, Y. H. Poortinga, M. H. Segall \& P. R. Dasen (Eds). Cross Cultural Psychology: Research and Application (pp. 42-68). New York, U.S.: Cambridge University Press.

Berry, J. W., Poortinga, Y. H., Segall, M. H. \& Dasen, P. R. (2002a). Approaches from cultural anthropoloty. In J. W. Berry, Y. H. Poortinga, M. H. Segall \& P. R. Dasen (Eds). Cross Cultural Psychology: Research and Application (pp. 225253). New York, U.S.: Cambridge University Press.

Berry, J. W., Poortinga, Y. H., Segall, M. H. \& Dasen, P. R. (2002b). Methodological 
concerns. In J. W. Berry, Y. H. Poortinga, M. H. Segall \& P. R. Dasen (Eds.). Cross Cultural Psychology: Research and Application (pp. 286-315). New York, U.S.: Cambridge University Press.

Berry, J. W., Poortinga, Y. H., Segall, M. H. \& Dasen, P. R. (2002c). Introduction to cross-cultural psychology. In J. W. Berry, Y. H. Poortinga, M. H. Segall \& P. R. Dasen (Eds.). Cross Cultural Psychology: Research and Application (pp. 1-18). New York, U.S.: Cambridge University Press.

Boeckmann, R. J. \& Tyler, T. R. (2003). Trust, respect and the psychology of political engagement. Journal of Applied Social Psychology, 32, 2067-2088.

Bohnet, I. \& Zeckhauser, R. (2004). Trust, risk and betrayal. Journal of Economic Behavior \& Organization, 55, 467-484.

Brandstrom, S., Richter, J. \& Przybeck, T. (2002). Distributions by age and sex of the dimensions of temperament and character inventory in cross-cultural perspective among Sweden, Germany, and the USA. Psychological Reports, 89, 747-758.

Brann, P., \& Foddy, M. (1987). Trust and the consumption of a deteriorating common resource. Journal of Conflict Resolustion, 31, 615-630

Brewer, M. B. (1999). The psychology of prejudice: In-group love or out-group hate? Journal of Social Issues, 55, 429-444.

Brewer, M. B. \& Roccas, S. (2001). Individual values, social identity, and optimal distinctiveness. In C. Sedikides \& M. B. Brewer (Eds.). Individual Self, Relational Self, Collective Self(pp. 219-240). New York, US: Psychology Press. Brewer, P., Aday, S. \& Gross, K. (2005). Do Americans trust other nations? A panel study. Social Science Quarterly, 86, 36-51. 
Brown, R. (2000). Social identity theory: Past achievements, current problems and future challenges. European Journal of Social Psychology, 30, 745-778.

Buchan, N. R. (2005). A worldwide exploratory inquiry into the influence of globalization on cooperation and trust. Proposal for National Science Foundation, Human and Social Development Program, Cross-directorate Activities, International Program.

Buchan, N. Croson, R. (2004). The boundaries of trust: Own and others' actions in the US and China. Journal of Economic Behavior and Organization, 55, 485-504.

Buchan, N. R., Croson, R. T. \& Dawes, R. M. (2002). Swift neighbors and persistent strangers: A cross-cultural investigation of trust and reciprocity in social exchange. American Journal of Sociology, 108, 168-206.

Butler, J. K. (1999). Trust expectations, information sharing, climate of trust, and negotiation effectiveness and efficacy. Coup and Organization Management, 24, 217-239.

Chen, C. C. Chen, Y. R. \& Xin, K. (2004). Guanxi Practices and Trust in Management: A Procedural Justice Perspective. Organization Science, 15, 200-209.

Child, J., \& Mollering, G. (2003). Contextual confidence and active trust development in the Chinese business environment. Organization Science, 14, 69-80.

Chirkov, V., Lynch, M. \& Niwa, S. (2005). Application of the scenario questionnaire of horizontal and vertical individualism and collectivism to the assessment of cultural distance and cultural fit. International Journal of Intercultural Relations, $29,469-490$.

Chou, L. F., Cheng, B. S, Huang, M. P, \& Cheng, H. Y. (2006). Guanxi networks and 
members' effectiveness in Chinese work teams: Mediating effects of trust networks. Asian Journal of Social Psychology, 9, 79-95.

Chow, I. H., Ng, I. (2004). The characteristics of Chinese personal ties (guanxi): Evidence from Hong Kong. Organization Studies, 25, 1075-1093.

Clarke, M. C., Heaton, M. B., Israelson, C. L. \& Eggett, D. L. (2005). The Acquisition of family financial roles and responsibilities. Family \& Consumer sciences Research Journal, 33, 321-340.

Cook, K. S. (2001). Trust in Society. Russell Sage Foundation Series on Trust, vol. 2. New York, US: Russell Sage Foundation.

Cook, K. S. \& Cooper, R. M. (2003). Experimental studies of cooperation, trust, and social exchange. In E. Ostrom, \& J. Walker (Eds.). Trust and Reciprocity: Interdisciplinary Lessons from Experimental Research (pp. 209-244). New York, US: Russell Sage Foundation.

Cook, K. S. \& Hardin, R. (2001). Norms of cooperativeness and networks of trust. In M. Hechter \& K. D. Opp (Eds.) Social Norma (pp. 327-347). New York, US: Russell Sage Foundation.

Cook, K. S., Hardin, R. \& Levi, M. (2005). Cooperation Without Trust? The Russell Sage Foundation Series on Trust. New York, US: Russell Sage Foundation.

Cook, K. S., Kramer, R. M., Thom, D. H., Stepanikova, I., Mollborn, S. B. \& Cooper, R. M. (2004). Trust and distrust in patient-physician relationships: perceived determinant of high and low trust relationships in managed care settings. In R. M. Kramer \& K. Cook (Eds.). Trust and Distrust in Organizations: Dilemmas and Approaches (pp. 65-98). New York, US: Russell Sage Foundation. 
Cook, K. S., Yamagishi, T. Cheshire, C., Cooper, R. Matsuda, M. \& Mashima, R. (2005). Trust building via risk taking: a cross-societal experiment. Social Psychology Quarterly, 68, 121-142.

Cooper, R. N. (2005). Whither China? Japan Center for Economic Research Bulletin, September, 1-11.

Coulson, N. S. (2002). Source of food safety information: Whom do adolescents trust? Appetite, 38, 199-200.

Cvetkovich, Siegrist, Murray, \& Tragesser, (2002). New information and social trust: Asymmetry and perseverance of attributions about hazard managers. Risk Analysis, 22, 359-367.

Dasgupta, P. (1988). Trust as a commodity. In D. Gambetta (Ed.) Trust: Making and Breaking cooperative Relations (pp. 49-71). Oxford: Basil Blackwell.

Dirks, K. T. (1999). The effects of interpersonal trust on work group performance. Journal of Applied Psychology, 84, 445-455. .

Ditommaso, E., Brannen, C. \& Burgess, M. (2005). The universality of relationship characteristics: A cross-cultural comparison of different types of attachment and loneliness in Canadian and visiting Chinese students. Social Behavior and Personality, 33, 57-68.

Draeger, C. (1969). Level of trust in intensive small groups. Dissertation Abstracts International, 29(10-A): 3457.

Earle, T. C. \& Cvetkorich, G. T. (1995). Social trust: Towards a cosmopolitan society. Westport, CT: Praeger Publishers.

Endo, Y., Heine, S-J. \& Lehman, D-R. (2000). Culture and positive illusions in close 
relationships: How my relationships are better than yours. Personality and Social Psychology Bulletin, 26, 1571-1586.

Engelbrecht, P. \& Natzel, S. G. (1997). Cultural variations in cognitive style: Field dependence vs. field independence. School Psychology International, 18, 155164.

Farh, J. L., Tsui, A. S., Xin, K. \& Cheng, B. S. (1998). The influence of relational demography and guanxi: The Chinese case. Organization Science, 9, 471-488.

Fijneman, Y., Willemsen, M., \& Poortinga, Y.H. (1996). Individualism-collectivism. An empirical study of a conceptual issue. Journal of Cross-cultural Psychology, 27, 381-402.

Fisler, C. (2005). Building trust and managing risk: A look at a felony mental health court. Psychology, Public Policy and Law, 11, 587-604.

Foddy, M. (2002). Group-based trust and cooperation in two interdependent settings. Society of Experimental Social Psychology. Columbus, Ohio.

Foddy, M. (2003). Rethinking reputation: Group membership and trustworthiness. Third CEFOM/21 International Symposium, Sapporo, Japan.

Foddy, M. Yamagishi, T., \& Platow, M.J. (2006) Stereotypes and expectations in groupbased trust. Under review.

Fukuyama, F (1995). Trust: The social virtues and the creation of prosperity. New York, U.S.: Free Press.

Gill, H., Boies, K., Finegan, J. E., \& McNally J. (2005). Antecedents of trust: Establishing a boundary condition for the relation between propensity to trust and intention to trust. Journal of Business and Psychology, 19, 287-302. 
Guttmann, J. (1993). Adolescents from divorced families and their best-friend relationship: A qualitative analysis. Journal of Divorce and Remarriage, 20, 95110.

Hardin, R. (2001). Conceptions and Explanations of Trust. In K. S. Cook (Ed.) Trust in Society, Volume II in the Russell Sage Foundation Series on Trust. New York, U.S.: Russell Sage Foundation.

Hardin, R. (2002). Trust and trustworthiness. New York, U.S.: Russell Sage Foundation.

Hofstede, G. (1980). Culture's consequence: International differences in work-related value. Beverly Hills: Sage

Hogg, M. A. (2001). Social identity and the sovereignty of the group: A psychology of belonging. In C. Sedikides \& M. B. Brewer (Eds.) Individual Self, Relational Self, and Collective Self(p.123-143). New York, U.S.: The Solomon R. Guggenheim Foundation.

Hoorens, V. \& Poortinga, Y. (2000). Behavior in social context. In K. Pawlik \& M. Rosenzweig (Eds.), International Handbook of Psychology (pp.40-63). London, U.K.: Sage.

Houlette, M.A., Gaertner, S. L., Johnson, K. M., Banker, B. S., Riek, B. M. \& Dovidio, J. F. (2004). Developing a more inclusive social identity: An elementary school intervention. Journal of Social Issues, 35-56.

Huff, L. \& Kelley, L. (2003). Levels of organizational trust in individualist versus collectivist societies: A seven-nation study. Organization Science, Special Issue: Trust in an Organization Content, 14, 81-90.

Huff, L. \& Kelley, L. (2005). Is collectivism a liability? The impact of culture on 
organizational trust and customer orientation: a seven-nation study. Journal of Business Research, 58, 96-102.

Hui, C. H. \& Yee, C. (1994). The shortened individualism-collectivism scale: Its relationship to demographic and work-related variables. Journal of Research in Personality, 28, 409-424.

Hwang, K-K (1997). Guanxi and Mientze: Conflict resolution in Chinese society. Intercultural Communication Studies VII: 1, 17-42.

Hwang, K. K. (1999). Filial piety and loyalty: Two types of social identification in confucianism. Asian Journal of Social Psychology, 2, 163-183.

Inglehart, R., Basanez, M. \& Moreno, A. (1998). Human cultures and beliefs: A crosscultural sourcebook: Political, religious, sexual, and economic Norms in 43 societies: Findings from the 1990-1993 World Values Survey. Ann Arbor: University of Michigan Press,

Jahoda, G. (1980). Theoretical and systematic approaches in cross-cultural psychology. In H. C. Triandis \& W. W. Lambert (Eds.) Handbook of Cross-cultural Psychology: Perspectives, Volume 1 (1-13). Boston, U.S.: Allyn and Bacon, INC. Jenkins, G. D. \& Lawler, E. E. (1981). Impact of employee participation in pay plan development. Organizational Behavior and Human Performance, 28, 111-128.

Jiang, X, Chaiwan, S. Panuthai, S., Yiang, C., Lei, Y. \& Li, Jiping (2002). Family support and self-care behavior of Chinese chronic obstructive pulmonary disease patients. Nursing and Health Sciences, 14, 41-49.

Kagitcibasis, C. (1997). Individualism and collectivism. In J. W. Berry, M. H. Segall, \& C. Kagitcibasi (Eds.), Social behavior and applications Vol.III of Handbook of 
Cross-cultural psychology (pp.1-49). Boston, MA: Allyn and Bacon.

Kashima, Y. (2002). Culture and self: A cultural dynamical analysis. In Y. Kashima, M. Foddy \& M. Platow (Eds.), Self and Identity: Personal, Social, and Symbolic (pp. 207-226). Mahwah, NJ, U.S.: Lawrence Erlbaum.

Kashima, Y. \& Foddy, M. (2002). Time and self: the historical construction of the self. In Y. Kashima, M. Foddy \& M. Platow (Eds.), Self and Identity: Personal, Social, and Symbolic (pp. 181-206). Mahwah, NJ, U.S.: Lawrence Erlbaum.

Kashima, Y. \& Triandis, H. C. (1986). The self-serving bias in attributions as a coping strategy: A cross-cultural study. Journal of Cross Cultural Psychology, 17, 83-97.

Kenrick, D. T. \& Trost, M. R. (2000). An evolutionary perspective on human Relationships. In W. Ickes \& S. Duck (Eds.). The Social Psychology of Personal Relationships. Chichester, England: John Wiley \& Sons Ltd.

Kerr, M. Stattin, H. \& Trost, K. (1999). To know you is to trust you: Parents' trust is rooted in child disclosure of information. Journal of Adolescence, 22, 737-752.

Kerr, R. \& Normand, R. (1992). Independent living and psychomotor performance. Canadian Journal on Aging, 11, 92-100.

Kewicki, R. J. \& Bunker,B. B. (1996). Developing and maintaining trust in work relationships. In R. M. Kramer \& T. R. Tyler (Eds.). Trust in Organizations: Frontiers of theory and Research (pp. 114-139). Thousand Oaks, CA, US: Sage publications, Inc.

Kim, U. Helgesen, G. \& Ahn, B. M. (2002). Democracy, trust, and political efficacy: Comparative analysis of Danish and Korean political cultural. Applied Psychology: An International Review, Special issue on Psychology in the Far 
East, Singapore, 51, 318-353.

Kitayama, S., Markus, H. R., Matsumoto, H., \& Norasakkunkit, V. (1997). Individual and collective processes in the construction of the self: Self-enhancement in the United States and Self-criticism in Japan. Journal of Personality and Social Psychology, 72, 1245-1267.

Kiyonari, T., Tanida, S. \& Yamagishi, T. (2000). Social exchange and reciprocity: Confusion or a heuristic? Evolution and Human Behavior, 21, 411-429.

Kiyonari, T. \& Yamagishi, T. (1999). A comparative study of trust and trustworthiness using the game of enthronement. Japanese Journal of Social Psychology, 15, 100109.

Kramer, R. M. (1996). Divergent realities and convergent disappointments in the hierarchic relation: Trust and the intuitive auditor at work. In R. M. Kramer, T. R. Tyler (Eds.). Trust in Organization: Frontiers of Theory and Research (pp. 216245). Thousand Oaks, U.S.: Sage Publications Inc.

Lewis, G. \& Weigert, A. (1985). Trust as a social reality. Social Forces, 63, 967-985.

Li, L \& Tracy, M. B. (2000). Family support, financial needs, and health care needs of rural elderly in China: A field study. Journal of Cross Cultural Gerontology, 14, 357-371.

Logan, J. R., Bian, F. (2003). Parents' needs, family structure, and regular intergenerational financial exchange in Chinese cities. Sociological Forum, 18, 85-101.

Luo, Y. (2002). Building trust in cross-cultural collaborations: Toward a contingency perspective. Journal of Management, 28, 669-694. 
Maddux, W. \& Brewer, M. (2005). Gender differences in the relational and collective bases for trust. Group Processes \& Intergroup Relations, 8, 159-171.

Maguire, S., Phillips, N. \& Hardy, C. (2001). When 'silence = death,' keep talking: Trust, control and the discursive construction of identity in the Canadian HIV/AIDS treatment domain. Organization Studies, 22, 285-310.

Markus, H. R. \& Kitayama, S. (2003). Culture, self, and the reality of the social. Psychological Inquiry, 14, 277-283.

Mastlund, E., Norlander, T. \& Archer, T. (2001). Exploring cross-cultural differences in self-concept: A meta analysis of the self-description questionnaire. Cross Cultural Research: The Journal of Comparative Social Science, 35, 280-302.

Mayer, R. C., Davis, J. H. \& Schoolman, F. D. (1995). An integrative model of organizational trust. Academy of Management Review, 20, 709-734.

McAllister, D. J. (1995). Affect and Cognition-Based trust as foundation for interpersonal cooperation in organizations. Academy of Management Journal, 38, 24-59.

Miller, R. L. \& Cangemi, J. P. (1988). Developing trust in international companies. Organization Development Journal, summer, 26-28.

Morrison, E. W. \& Robinson. S. L. (1997). When employees feel betrayed: A model of how psychological contract violation develops. Academy of Management Review, 22, 226-256.

Munroe, R. H. \& Munroe, R.L. (1994). Field observations of behavior as a cross-cultural method. In P.K. Bock (Ed.), Handbook of Psychological Anthropology (pp. 255271). Westport, CT: Greenwood.

Munroe, R. L. \& Munroe, R. L. (1997). A comparative antheopological perspective. In J. 
W. Berry, Y. H. Poortinga \& J. Pandey (Eds.), Theory and Method Vol. I of Handbook of Cross cultural Psychology (pp.171-213). Boston, MA:

Allyn and Bacon.

Nisbett, R. E., Peng, K., Choi, I. \& Norenzayan, A. (2001). Culture and systems of thought: Holistic versus analytic cognition. Psychological Review, 108, 291-310.

Niu, J. (1995). The development of the emotional experience aged 5-11 years in Dai and Han nationalities. Psychological Development and Education, vol. 11, no. 3, 1420.

Ontario Ministry of Training, Colleges and Universities (2005). Ontario student assistance program. Ottawa, Queens Printer for Ontario.

Packer, M. J. \& Tappan, M. B. (2001). Introduction. In M. Packer \& M. Tappan (Eds.) Cultural and Critical Perspectives on Human Development. (p1-p40.). Albanny, NY: State University of New York Press.

Pei, C. \& Pillai, E. (1999). Old age support in China: The role of the state and the family. International Journal of Aging and Human Development, 49, 197-212.

Peng, S. (2001). Guanxi-management and legal approaches to establish and enhance interpersonal trust. Journal of Psychology in Chinese Societies, 2, 51-76.

Phelps, R. E., Taylor, J. D. \& Gerard, P. A. (2001). Cultural mistrust, ethnic identity, racial identity, and self-esteem among ethnically diverse black students. Journal of Counselling and Development, 79, 209-216.

Porter, G. (1997). Trust in teams: Member perceptions and the added concern of crosscultural interpretations. Advances in Interdisciplinary Studies of Work Teams, 4, 45-77. 
Probst, T. \& Lawler, J. (2006). Cultural values as moderators of employee reactions to job insecurity: the role of individualism and collectivism. Applied Psychology: An International Review, 55, 234-254.

Putnam, R. D. (1995). Bowling alone: America's declining social capital. Journal of Democracy, 6, 65-78.

Ramasamy, G. Goh, K. W. \& Yeung, M. C. (2006). Is Guanxi (relationship) a bridge to knowledge transfer? Journal of Business Research, 59, 130-139.

Rose, R. (1994). Post communism and problem of trust. Journal of Democracy, July, 1830.

Rotenberg, K., Boulton, M. J., Fox, C. L. (2005). Cross-Sectional and Longitudinal Relations Among Children's Trust Beliefs, Psychological Maladjustment and Social Relationships: Are Very High as Well as Very Low Trusting Children at Risk? Journal of Abnormal Child Psychology, 33, 595-610.

Rotter, J. B. (1967). A new scale for the measurement of interpersonal trust. Journal of Personality 35, 615-665.

Rousseau, D. M., Sitkin, S. B., Burt, R. S., \& Camerer, C. (1998). Not so different after all: A cross-discipline view of trust. Academy of Management Review 23, 393404.

Sakai, A., Sugawara, M., Maeshiro, K., Sugawara, K. \& Kitamura, T. (2002). Parentchild relations of mutual trust, trust in one's best friend and school adjustment: Junior high school students. Japanese Journal of Educational Psychology, 50, 1222.

Sares, T. A. (1998). Sociopolitical viewpoints as narrated by family and educational 
background. Journal of social Psychology, 138, 637-644.

Sato, K. (1988). Trust and group size in a social dilemma. Japanese Psychological Research, 30, 88-93.

Schnittker, J. (2004). Social distance in the clinical encounter: Interactional and sociodemographic foundations for mistrust in physicians. Social Psychology Quarterly, 67, 217-235.

Segall, M. H. (1979). The sociocultural nature of human beings. In M. H. Segall (Ed.) Cross-cultural Psychology: Human Behavior in Global Perspective. Belmont, AA: Wadsworth, Inc.

Segall, M. H. (1999). Why is there still racism if there is no such thing as "race"? In W. J. Lonner, D. L. Dinnel (Eds.) Merging Past, Present, and Future in Cross-cultural Psychology: Selected Papers from the Fourteenth International Congress of the International Association for Cross-cultural Psychology (pp. 14-26). Bristol, PA, US: Swets \& Zeitlinger Publishers.

Sheppard, B. H. \& Sherman, D. H. (1998). The grammars of trust: A model and general implications. Academy of Management Review, 23, 422-439.

Sztompka, P. (1999a). The turn toward soft variables in sociological theory. In P.

Sztompka (Ed.) Trust (1-17). Cambridge, U.K.: Cambridge University Press.

Sztompka, P. (1999b). The idea of trust. In P. Sztompka (Ed.), Trust (18-40). Cambridge, U.K.: Cambridge University Press.

Thiede, M. (2005). Information and access to health care: Is there a role for trust? Social Science and Medicine, 61, 1452-1462. 
Thye, S. R., Yoon, J. \& Lawler, E. J. (2002). The theory of relational cohesion: Review of a research program. In Thye, S. R. \& Lawyer, E. J. (Eds.), Group Cohesion, Trust and Solidarity (pp.139-166), Advances in Group Processes, 19. US: Elsevier Science/JAT Press.

Tilly, C (2005). Relations of trust and distrust. In C. Tilly (Ed.) Trust and Rule (1-29). New York, US: Cambridge University Press.

Triandis, H. C. (1980). Introduction to handbook of cross-cultural psychology. In H. C. Triandis \& W. W. Lambert (Eds.) Handbook of Cross-cultural Psychology: Perspectives, Volume 1 (pp. 1-13). Boston, U.S.: Allyn and Bacon, INC.

Trindis, H. C. (1988). Collectivism and individualism: A re-conceptualization of a basic concept in cross-cultural psychology. In G. K. Verma \& C. Bagley (Eds.), Personality, Attitudes and Cognitions (pp. 60-95). London: MacMilian.

Triandis, H. C. (1995). Individualism and collectivism. Boulder, CO: Westview. Triandis, H. C., Brislin, R. \& Hui, C. H. (1988). Cross-cultural training across the individualism collectivism divide. International Journal of Intercultural Relations, 12, 269-289.

Triandis, H. C. \& Trafimow, D. (2001). Cross national prevalence of collectivism. In C. Sedikides \& M. B. Brewer (Eds.). Individual Self, Relational Self, Collective Self (pp. 259-276). New York, US: Psychology Press.

Trompenaars, F. (1993). Riding the Waves of Culture. Economist Books, London, U.K. Tsui A.S. \& Farh J.L. (1997). Where Guanxi matters, Work Occup, 24, 56-79.

Turner, M. E. \& Pratkanis, A. R. (1998). A social identity maintenance model of groupthink. Organization Behavior and Human Decision Processes. 73, 
210-235.

Tyler, T. R. (2001). Why do people rely on others? Social identity and the social aspects of trust. In Karen S. Cook (Ed.) Trust in Society (pp.285-306). New York, U.S.: Russell Sage Foundation.

Tyler, T. R. (2003a). Trust within organization. Personnel Review, 32, 556-568.

Tyler, T. R (2003b). Identification and Cooperation in Groups. Third CEFOM/21 International Symposium. Sapporo, Japan.

Tyler, T. R. \& Huo, Y. J. (2002). Trust in the law: Encouraging public cooperation with the police and courts. New York, U.S.: Russell Sage Foundation.

Wang, K. Y. \& Clegg, S. (2002). Trust and decision making: Are managers different in the People's Republic of China and in Australia? Cross Cultural Management, 9, $30-45$.

Wang, F. \& Yamagishi, T. (2005). Group-based trust and gender differences in China. Asian Journal of Social Psychology, 8, 199-210.

Watkins, D., Mortazavi, S. \& Troffmova, I. (2000). Independent and interdependent conceptions of self: An investigation of age, gender, and culture differences in importance and satisfaction ratings. The Journal of Comparative Social Science, 34, 113-114.

Welch, M., Rivera, R. E. Conway, B. P., Yonkoski, J. Lupton, P. M. \& Giancola, R. (2005). Determinants and consequences of social trust. Sociological Inquiry, 75, $453-473$.

Whitener, E. M. (2006). The impact of human resource activities on employee trust. Human Resource Management Review, 7, 389-404. 
Williamson, O. E. (1993). Calculativeness, trust and economic organization. Journal of Law and Economics, 30, 131-145.

Yamagishi, T. (1988). The provision of a sanctioning system in the United States and Japan. Social Psychology Quarterly, 51, 265-271.

Yamagishi, T. (2003). An institutional approach to groups. Third CEFOM/21 International Symposium. Sapporo, Japan.

Yamagishi, T., Cook, K. S., Watabe, M. (1998). Uncertainty, trust, and commitment formation in the United States and Japan. American Journal of Sociology, 104, 165-194.

Yamagishi, T., Makimura, Y., Foddy, M. Matsuda, M. Kiyonari, T. \& Platow, M. (2005). Comparisons of Australians and Japanese on group-based cooperation. Asian Journal of Social Psychology, 8, 173-190.

Yamagishi, T., \& Yamagishi, M. (1994). Trust and commitment in the United States and Japen. Motivation and Emotion, 18, 129-166.

Yoshino, R. (2002). A time to trust: A study on people's sense of trust from a viewpoint of cross-national and longitudinal study on national character. Behaviormetrika, $29,231-260$.

Yuki, M. (2003). Category-based collectivism versus network-based collectivism: identifying two types of individual-group relations in the west and east. Third CEFOM/21 International Symposium, Sapporo, Japan.

Yuki, M. Maddux, W. W. Brewer, M. B. \& Takemura, K. (2005). Cross-cultural difference in relationship and group-based trust. Personality and Social Psychology Bulletin, 31, 48-62. 
Zhang, B. (2005). China Educational Department $11^{\text {th }}$ release news of 2005. Retrieved October 3, 2005 from http://www.edu.cn/20050829/3148861.shtml\#2

Zhang, J. \& Bond, M. H. (1993). Target-based interpersonal trust: Cross-cultural comparison and its cognitive model. Acta Psychologica Sinica, 25, 164-172.

Zhang, L. (2005). Prediction of Chinese life satisfaction: Contribution of collective selfesteem. International Journal of Psychology, 40, 189-200. 
Appendix A: When it Comes to Economic Matters, Whom Would You Trust? 


\section{When it Comes to Economic Matters, WHOM Would You Trust?}

Instructions: The purpose of this questionnaire is to get related data about trust. The information you provide is totally anonymous and confidential. In addition, there are no right or wrong answers or good or bad answers. Please express your own thoughts, ideas, and feelings, not what you think is generally desirable. Thank you very much for your participation.

1. Whom, do you think, you would trust to lend $\$ 10$ to, if possible? Choose possible person(s) from the following list, and place an $\mathrm{X}$ within the corresponding small box.

\begin{tabular}{|l|l|l|l|}
\hline Acquaintance /Neighbor & 1 & Grandparents & 8 \\
\hline $\begin{array}{l}\text { Bank manager/Accountant } \\
\text { Best/close friend }\end{array}$ & 2 & $\begin{array}{l}\text { Husband (wife) /Boyfriend } \\
\text { (girlfriend) /Partner /Lover }\end{array}$ & 9 \\
\hline Boss /Supervisor/Teacher & 3 & Parents /Guardian & 10 \\
\hline Children /Grandchildren & 4 & Psychologist/Professional counselor & 11 \\
\hline Classmate/Co-worker/Colleague & 6 & Relative & 12 \\
\hline Friend & 7 & Stranger & 13 \\
\hline
\end{tabular}

2. Whom, do you think, you would trust to lend $\$ 500$ to, if possible? Choose possible person(s) from the following list, and place an $\mathrm{X}$ within the corresponding small box.

\begin{tabular}{|l|l|l|l|}
\hline Acquaintance/Neighbor & 1 & Grandparents & 8 \\
\hline Bank manager/Accountant & 2 & $\begin{array}{c}\text { Husband (wife)/Boyfriend } \\
\text { (girlfriend) /Partner /Lover }\end{array}$ & 9 \\
\hline Best/close friend & 3 & Parents/Guardian & 10 \\
\hline Boss /Supervisor/Teacher & 4 & Psychologist/Professional counselor & 11 \\
\hline Children /Grandchildren & 5 & Relative & 12 \\
\hline Classmate/Co-worker/Colleague & 6 & Sibling & 13 \\
\hline Friend & 7 & Stranger & 14 \\
\hline
\end{tabular}


3. Whom, do you think, you would trust to lend $\$ 10,000$ to, if possible? Choose possible person(s) from the following list, and place an $\mathrm{X}$ within the corresponding small box.

\begin{tabular}{|l|l|l|l|}
\hline Acquaintance /Neighbor & 1 & Grandparents & 8 \\
\hline Bank manager/Accountant & 2 & $\begin{array}{l}\text { Husband (wife)/Boyfriend } \\
\text { (girlfriend)/Partner/Lover }\end{array}$ & 9 \\
\hline Best/close friend & 3 & Parents/Guardian & 10 \\
\hline Boss /Supervisor/Teacher & 4 & Psychologist/Professional counselor & 11 \\
\hline Children/Grandchildren & 5 & Relative & 12 \\
\hline Classmate/Co-worker/Colleague & 6 & Sibling & 13 \\
\hline Friend & 7 & Stranger & 14 \\
\hline
\end{tabular}

4. Whom, do you think, you would trust to lend $\$ 100,000 \&$ more to, if possible? Choose possible person(s) from the following list, and place an $\mathrm{X}$ within the corresponding small box.

\begin{tabular}{|l|l|l|l|}
\hline Acquaintance/Neighbor & 1 & Grandparents & 8 \\
\hline Bank manager /Accountant & 2 & $\begin{array}{l}\text { Husband (wife) /Boyfriend } \\
\text { (girlfriend)/Partner /Lover }\end{array}$ & 9 \\
\hline Best/close friend & 3 & Parents/Guardian & 10 \\
\hline Boss/Supervisor/Teacher & 4 & Psychologist/Professional counselor & 11 \\
\hline Children/Grandchildren & 5 & Relative & 12 \\
\hline Classmate/Co-worker/Colleague & 6 & Sibling & 13 \\
\hline Friend & 7 & Stranger & 14 \\
\hline
\end{tabular}


Appendix B: When it Comes to Positive Matters, Whom Would You Trust? 


\section{When it Comes to Positive Matters, WHOM Would You Trust?}

Instructions: The purpose of this questionnaire is to get related data about trust. The information you provide is totally anonymous and confidential. In addition, there are no right or wrong answers or good or bad answers. Please express your own thoughts, ideas, and feelings, not what you think is generally desirable. Thank you very much for your participation.

1. Whom, do you think, you would trust to share the information with, if you had good comments on your assignment/homework? Choose possible person(s) from the following list and place an $\mathrm{X}$ within the corresponding small box.

\begin{tabular}{|l|l|l|l|}
\hline Acquaintance /Neighbor & 1 & Grandparents & 8 \\
\hline Bank manager /Accountant & 2 & $\begin{array}{l}\text { Husband (wife)/Boyfriend } \\
\text { (girlfriend) /Partner/Lover }\end{array}$ & 9 \\
\hline Best/close friend & 3 & Parents/Guardian & 10 \\
\hline Boss /Supervisor/Teacher & 4 & Psychologist/Professional counselor & 11 \\
\hline Children /Grandchildren & 5 & Relative & 12 \\
\hline Classmate/Co-worker/Colleague & 6 & Sibling & 13 \\
\hline Friend & 7 & Stranger & 14 \\
\hline
\end{tabular}

2. Whom, do you think, you would trust to share the information with, if you got a good mark/grade on your test/examination? Choose possible person(s) from the following list and place an $\mathrm{X}$ within the corresponding small box.

\begin{tabular}{|l|l|l|l|}
\hline Acquaintance /Neighbor & 1 & Grandparents & 8 \\
\hline Bank manager/Accountant & 2 & $\begin{array}{c}\text { Husband (wife)/Boyfriend } \\
\text { (girlfriend) /Partner /Lover }\end{array}$ & 9 \\
\hline Best/close friend & 3 & Parents /Guardian & 10 \\
\hline Boss /Supervisor/Teacher & 4 & Psychologist/Professional counselor & 11 \\
\hline Children /Grandchildren & 5 & Relative & 12 \\
\hline Classmate /Co-worker/Colleague & 6 & Sibling & 13 \\
\hline Friend & 7 & Stranger & 14 \\
\hline
\end{tabular}


3. Whom, do you think, you would trust to share the information with, if you were the best student in your class? Choose possible person(s) from the following list and place an $\mathrm{X}$ within the corresponding small box.

\begin{tabular}{|c|c|c|c|}
\hline Acquaintance $/$ Neighbor & 1 & Grandparents & 8 \\
\hline Bank manager/Accountant & 2 & $\begin{array}{l}\text { Husband (wife) /Boyfriend } \\
\text { (girlfriend) /Partner/Lover }\end{array}$ & 9 \\
\hline Best/close friend & 3 & Parents /Guardian & 10 \\
\hline Boss /Supervisor /Teacher & 4 & Psychologist/Professional counselor & 11 \\
\hline Children/Grandchildren & 5 & Relative & 12 \\
\hline Classmate /Co-worker/Colleague & 6 & Sibling & 13 \\
\hline Friend & 7 & Stranger & 14 \\
\hline
\end{tabular}

4. Whom, do you think, you would trust to share the information with, if you were the best student in your school/university? Choose possible person(s) from the following list and place an $\mathrm{X}$ within the corresponding small box.

\begin{tabular}{|l|l|l|l|}
\hline Acquaintance /Neighbor & 1 & Grandparents & 8 \\
\hline Bank manager/Accountant & 2 & $\begin{array}{l}\text { Husband (wife)/Boyfriend } \\
\text { (girlfriend) /Partner /Lover }\end{array}$ & 9 \\
\hline Best/close friend & 3 & Parents/Guardian & 10 \\
\hline Boss /Supervisor/Teacher & 4 & Psychologist/Professional counselor & 11 \\
\hline Children /Grandchildren & 5 & Relative & 12 \\
\hline Classmate/Co-worker/Colleague & 6 & Sibling & 13 \\
\hline Friend & 7 & Stranger & 14 \\
\hline
\end{tabular}


Appendix C: When it Comes to Negative Matters, Whom Would You Trust? 


\section{When it Comes to Negative Matters, WHOM Would You Trust?}

Instructions: The purpose of this questionnaire is to get related data about trust. The information you provide is totally anonymous and confidential. In addition, there are no right or wrong answers or good or bad answers. Please express your own thoughts, ideas, and feelings, not what you think is generally desirable. Thank you very much for your participation.

1. Whom, do you think, you would trust to share the information with, if you had bad comments on your assignment/homework? Choose possible person(s) from the following list and place an $\mathrm{X}$ within the corresponding small box.

\begin{tabular}{|l|l|l|l|}
\hline Acquaintance/Neighbor & 1 & Grandparents & 8 \\
\hline Bank manager/Accountant & 2 & $\begin{array}{c}\text { Husband (wife)/Boyfriend } \\
\text { (girlfriend)/Partner/Lover }\end{array}$ & 9 \\
\hline Best/close friend & 3 & Parents/Guardian & 10 \\
\hline Boss/Supervisor/Teacher & 4 & Psychologist/Professional counselor & 11 \\
\hline Children/Grandchildren & 5 & Relative & 12 \\
\hline Classmate/Co-worker/Colleague & 6 & Sibling & 13 \\
\hline Friend & 7 & Stranger & 14 \\
\hline
\end{tabular}

2. Whom, do you think, you would trust to share the information with, if you got a bad mark/grade on your test/examination? Choose possible person(s) from the following list and place an $\mathrm{X}$ within the corresponding small box.

\begin{tabular}{|l|l|l|l|}
\hline Acquaintance /Neighbor & 1 & Grandparents & 8 \\
\hline Bank manager/Accountant & 2 & $\begin{array}{c}\text { Husband (wife)/Boyfriend } \\
\text { (girlfriend)/Partner /Lover }\end{array}$ & 9 \\
\hline Best/close friend & 3 & Parents /Guardian & 10 \\
\hline Boss/Supervisor/Teacher & 4 & Psychologist/Professional counselor & 11 \\
\hline Children/Grandchildren & 5 & Relative & 12 \\
\hline Classmate/Co-worker/Colleague & 6 & Sibling & 13 \\
\hline Friend & 7 & Stranger & 14 \\
\hline
\end{tabular}


3. Whom, do you think, you would trust to share the information with, if you failed your course/program? Choose possible person(s) from the following list and place an $\mathrm{X}$ within the corresponding small box.

\begin{tabular}{|l|l|l|l|}
\hline Acquaintance/Neighbor & 1 & Grandparents & 8 \\
\hline Bank manager/Accountant & 2 & $\begin{array}{l}\text { Husband (wife)/Boyfriend } \\
\text { (girlfriend)/Partner/Lover }\end{array}$ & 9 \\
\hline Best/close friend & 3 & Parents /Guardian & 10 \\
\hline Boss/Supervisor/Teacher & 4 & Psychologist/Professional counselor & 11 \\
\hline Children /Grandchildren & 5 & Relative & 12 \\
\hline Classmate/Co-worker/Colleague & 6 & Sibling & 13 \\
\hline Friend & 7 & Stranger & 14 \\
\hline
\end{tabular}

4. Whom, do you think, you would trust to share the information with, if you were expelled from your school/university? Choose possible person(s) from the following list and place an $\mathrm{X}$ within the corresponding small box.

\begin{tabular}{|l|l|l|l|}
\hline Acquaintance/Neighbor & 1 & Grandparents & 8 \\
\hline Bank manager/Accountant & 2 & $\begin{array}{c}\text { Husband (wife)/Boyfriend } \\
\text { (girlfriend)/Partner/Lover }\end{array}$ & 9 \\
\hline Best/close friend & 3 & Parents/Guardian & 10 \\
\hline Boss/Supervisor/Teacher & 4 & Psychologist/Professional counselor & 11 \\
\hline Children /Grandchildren & 5 & Relative & 12 \\
\hline Classmate/Co-worker/Colleague & 6 & Sibling & 13 \\
\hline Friend & 7 & Stranger & 14 \\
\hline
\end{tabular}


Appendix D: When it Comes to Economic Matters, Who Would Trust You? 


\section{When it Comes to Economic Matters, WHO Would Trust You?}

Instructions: The purpose of this questionnaire is to get related data about trust. The information you provide is totally anonymous and confidential. In addition, there are no right or wrong answers or good or bad answers. Please express your own thoughts, ideas, and feelings, not what you think is generally desirable. Thank you very much for your participation.

1. Who, do you think, would trust you to lend you $\$ 10$, if possible? Choose possible person(s) from the following list, and place an $\mathrm{X}$ within the corresponding small box.

\begin{tabular}{|l|l|l|l|}
\hline Acquaintance /Neighbor & 1 & Grandparents & 8 \\
\hline Bank manager/Accountant & 2 & $\begin{array}{c}\text { Husband (wife)/Boyfriend } \\
\text { (girlfriend) /Partner /Lover }\end{array}$ & 9 \\
\hline Best/close friend & 3 & Parents/Guardian & 10 \\
\hline Boss /Supervisor/Teacher & 4 & Psychologist/Professional counselor & 11 \\
\hline Children/Grandchildren & 5 & Relative & 12 \\
\hline Classmate/Co-worker/Colleague & 6 & Sibling & 13 \\
\hline Friend & 7 & Stranger & 14 \\
\hline
\end{tabular}

2. Who, do you think, would trust you to lend you $\$ 500$, if possible? Choose possible person(s) from the following list, and place an $\mathrm{X}$ within the corresponding small box.

\begin{tabular}{|c|c|c|c|}
\hline Acquaintance $/$ Neighbor & 1 & Grandparents & 8 \\
\hline Bank manager /Accountant & 2 & $\begin{array}{l}\text { Husband (wife) /Boyfriend } \\
\text { (girlfriend) /Partner /Lover }\end{array}$ & 9 \\
\hline Best/close friend & 3 & Parents /Guardian & 10 \\
\hline Boss /Supervisor/Teacher & $\overline{4}$ & Psychologist /Professional counselor & 11 \\
\hline Children /Grandchildren & 5 & Relative & 12 \\
\hline Classmate /Co-worker/Colleague & 6 & Sibling & 13 \\
\hline Friend & 7 & Stranger & 14 \\
\hline
\end{tabular}


3. Who, do you think, would trust you to lend you $\$ 10,000$, if possible? Choose possible person(s) from the following list, and place an $\mathrm{X}$ within the corresponding small box.

\begin{tabular}{|c|c|c|c|}
\hline Acquaintance / Neighbor & 1 & Grandparents & 8 \\
\hline Bank manager/Accountant & 2 & $\begin{array}{l}\text { Husband (wife) /Boyfriend } \\
\text { (girlfriend) /Partner /Lover }\end{array}$ & 9 \\
\hline Best/close friend & 3 & Parents /Guardian & 10 \\
\hline Boss /Supervisor /Teacher & 4 & Psychologist /Professional counselor & 11 \\
\hline Children /Grandchildren & 5 & Relative & 12 \\
\hline Classmate /Co-worker /Colleague & 6 & Sibling & 13 \\
\hline Friend & 7 & Stranger & 14 \\
\hline
\end{tabular}

4. Who, do you think, would trust you to lend you $\$ 100,000 \&$ more, if possible? Choose possible person(s) from the following list, and place an $\mathrm{X}$ within the corresponding small box.

\begin{tabular}{|l|l|l|l|}
\hline Acquaintance /Neighbor & 1 & Grandparents & 8 \\
\hline Bank manager/Accountant & 2 & $\begin{array}{c}\text { Husband (wife)/Boyfriend } \\
\text { (girlfriend) /Partner/Lover }\end{array}$ & 9 \\
\hline Best/close friend & 3 & Parents /Guardian & 10 \\
\hline Boss/Supervisor/Teacher & 4 & Psychologist/Professional counselor & 11 \\
\hline Children/Grandchildren & 5 & Relative & 12 \\
\hline Classmate/Co-worker/Colleague & 6 & Sibling & 13 \\
\hline Friend & 7 & Stranger & 14 \\
\hline
\end{tabular}


Appendix E: When it Comes to Positive Matters, Who Would Trust You? 


\section{When it Comes to Positive Matters, WHO Would Trust You?}

Instructions: The purpose of this questionnaire is to get related data about trust. The information you provide is totally anonymous and confidential. In addition, there are no right or wrong answers or good or bad answers. Please express your own thoughts, ideas, and feelings, not what you think is generally desirable. Thank you very much for your participation.

1. Who, do you think, would trust you to share the information with you, if he/she had good comments on his/her assignment/homework? Choose possible person(s) from the following list and place an $\mathrm{X}$ within the corresponding small box.

\begin{tabular}{|l|l|l|l|}
\hline Acquaintance/Neighbor & 1 & Grandparents & 8 \\
\hline Bank manager/Accountant & 2 & $\begin{array}{r}\text { Husband (wife)/Boyfriend } \\
\text { (girlfriend)/Partner/Lover }\end{array}$ & 9 \\
\hline Best/close friend & 3 & Parents/Guardian & 10 \\
\hline Boss /Supervisor/Teacher & 4 & Psychologist /Professional counselor & 11 \\
\hline Children/Grandchildren & 5 & Relative & 12 \\
\hline Classmate/Co-worker/Colleague & 6 & Sibling & 13 \\
\hline Friend & 7 & Stranger & 14 \\
\hline
\end{tabular}

2. Who, do you think, would trust you to share the information with you, if he/she got a good mark/grade on his/her test/examination? Choose possible person(s) from the following list and place an $\mathrm{X}$ within the corresponding small box.

\begin{tabular}{|l|l|l|l|}
\hline Acquaintance /Neighbor & 1 & Grandparents & 8 \\
\hline Bank manager /Accountant & 2 & $\begin{array}{c}\text { Husband (wife)/Boyfriend } \\
\text { (girlfriend)/Partner/Lover }\end{array}$ & 9 \\
\hline Best/close friend & 3 & Parents /Guardian & 10 \\
\hline Boss /Supervisor/Teacher & 4 & Psychologist/Professional counselor & 11 \\
\hline Children /Grandchildren & 5 & Relative & 12 \\
\hline Classmate/Co-worker/Colleague & 6 & Sibling & 13 \\
\hline Friend & 7 & Stranger & 14 \\
\hline
\end{tabular}


3. Who, do you think, would trust you to share the information with you, if he/she was the best student in his/her class? Choose possible person(s) from the following list and place an $\mathrm{X}$ within the corresponding small box.

\begin{tabular}{|c|c|c|c|}
\hline Acquaintance /Neighbor & 1 & Grandparents & 8 \\
\hline Bank manager /Accountant & 2 & $\begin{array}{l}\text { Husband (wife)/Boyfriend } \\
\text { (girlfriend)/Partner /Lover }\end{array}$ & 9 \\
\hline Best/close friend & 3 & Parents /Guardian & 10 \\
\hline Boss /Supervisor /Teacher & 4 & Psychologist/Professional counselor & 11 \\
\hline Children /Grandchildren & 5 & Relative & 12 \\
\hline Classmate/Co-worker/Colleague & 6 & Sibling & 13 \\
\hline Friend & 7 & Stranger & 14 \\
\hline
\end{tabular}

4. Who, do you think, would trust you to share the information with you, if he/she was the best student in his/her school/university? Choose possible person(s) from the following list and place an $\mathrm{X}$ within the corresponding small box.

\begin{tabular}{|c|c|c|c|}
\hline Acquaintance $/$ Neighbor & 1 & Grandparents & 8 \\
\hline Bank manager /Accountant & 2 & $\begin{array}{l}\text { Husband (wife) /Boyfriend } \\
\text { (girlfriend) /Partner /Lover }\end{array}$ & 9 \\
\hline Best/close friend & 3 & Parents /Guardian & 10 \\
\hline Boss /Supervisor /Teacher & 4 & Psychologist /Professional counselor & 11 \\
\hline Children /Grandchildren & 5 & Relative & 12 \\
\hline Classmate/Co-worker/Colleague & 6 & Sibling & 13 \\
\hline Friend & 7 & Stranger & 14 \\
\hline
\end{tabular}


Appendix F: When it Comes to Negative Matters, Who Would Trust You? 


\section{When it Comes to Negative Matters, WHO Would Trust You?}

Instructions: The purpose of this questionnaire is to get related data about trust. The information you provide is totally anonymous and confidential. In addition, there are no right or wrong answers or good or bad answers. Please express your own thoughts, ideas, and feelings, not what you think is generally desirable. Thank you very much for your participation.

1. Who, do you think, would trust you to share the information with you, if he/she had bad comments on his/her assignment/homework? Choose possible person(s) from the following list and place an $\mathrm{X}$ within the corresponding small box.

\begin{tabular}{|l|l|l|l|}
\hline Acquaintance /Neighbor & 1 & Grandparents & 8 \\
\hline Bank manager/Accountant & 2 & $\begin{array}{c}\text { Husband (wife)/Boyfriend } \\
\text { (girlfriend) /Partner /Lover }\end{array}$ & 9 \\
\hline Best/close friend & 3 & Parents /Guardian & 10 \\
\hline Boss /Supervisor/Teacher & 4 & Psychologist/Professional counselor & 11 \\
\hline Children/Grandchildren & 5 & Relative & 12 \\
\hline Classmate/Co-worker/Colleague & 6 & Sibling & 13 \\
\hline Friend & 7 & Stranger & 14 \\
\hline
\end{tabular}

2. Who, do you think, would trust you to share the information with you, if he/she got a bad mark/grade on his/her test/examination? Choose possible person(s) from the following list and place an $\mathrm{X}$ within the corresponding small box.

\begin{tabular}{|l|l|l|l|}
\hline Acquaintance /Neighbor & 1 & Grandparents & 8 \\
\hline Bank manager /Accountant & 2 & $\begin{array}{c}\text { Husband (wife)/Boyfriend } \\
\text { (girlfriend) /Partner /Lover }\end{array}$ & 9 \\
\hline Best/close friend & 3 & Parents/Guardian & 10 \\
\hline Boss /Supervisor/Teacher & 4 & Psychologist/Professional counselor & 11 \\
\hline Children /Grandchildren & 5 & Relative & 12 \\
\hline Classmate /Co-worker/Colleague & 6 & Sibling & 13 \\
\hline Friend & 7 & Stranger & 14 \\
\hline
\end{tabular}


3. Who, do you think, would trust you to share the information with you, if he/she failed his/her course/program? Choose possible person(s) from the following list and place an X within the corresponding small box.

\begin{tabular}{|l|l|l|l|}
\hline Acquaintance /Neighbor & 1 & Grandparents & 8 \\
\hline Bank manager/Accountant & 2 & $\begin{array}{c}\text { Husband (wife) /Boyfriend } \\
\text { (girlfriend) /Partner/Lover }\end{array}$ & 9 \\
\hline Best/close friend & 3 & Parents/Guardian & 10 \\
\hline Boss/Supervisor/Teacher & 4 & Psychologist/Professional counselor & 11 \\
\hline Children /Grandchildren & 5 & Relative & 12 \\
\hline Classmate/Co-worker/Colleague & 6 & Sibling & 13 \\
\hline Friend & 7 & Stranger & 14 \\
\hline
\end{tabular}

4. Who, do you think, would trust you to share the information with you, if he/she was expelled from his/her school/university? Choose possible person(s) from the following list and place an $\mathrm{X}$ within the corresponding small box.

\begin{tabular}{|c|c|c|c|}
\hline Acquaintance /Neighbor & 1 & Grandparents & 8 \\
\hline Bank manager/Accountant & 2 & $\begin{array}{l}\text { Husband (wife) /Boyfriend } \\
\text { (girlfriend) /Partner /Lover }\end{array}$ & 9 \\
\hline Best/close friend & 3 & Parents /Guardian & 10 \\
\hline Boss /Supervisor /Teacher & 4 & Psychologist /Professional counselor & 11 \\
\hline Children /Grandchildren & 5 & Relative & 12 \\
\hline Classmate /Co-worker /Colleague & 6 & Sibling & 13 \\
\hline Friend & 7 & Stranger & 14 \\
\hline
\end{tabular}


Appendix G (Chinese version): When it Comes to Economic Matters, Whom Would You Trust? 


\section{在涉及经济物质的情况下，}

\section{你将信任谁?}

兑明: 这份问卷的目的是为信任研究收集资料。和所有其他的参与者一样, 你所提供的信 息完全是不署名的和保密的。对问题的回答也完全没有任何好坏、对错之分。请表达出你 自己的想法和感受，而不是其它。非常感谢你对本研究的支持。

1. 你觉得你会信任并借给谁10块钱, 如果可能的话? 在以下的备选答案中选出你 的答案, 并在相应的小框内打勾。

\begin{tabular}{|c|c|c|c|}
\hline 熟人ノ邻居 & 1 & 爷爷好奶／外公外婆 & 8 \\
\hline 银行经理/会计 & 2 & 丈夫(妻子)/男朋友(文朋友)/情侣 & 9 \\
\hline 最好／最亲密的朋友 & 3 & 父母／监护人 & 10 \\
\hline 老板／领导／老师 & 4 & 心理学家/专业咨洵师 & 11 \\
\hline 子女文 /孙子㚣 & 5 & 亲成 & 12 \\
\hline 同学／工友／同事 & 6 & 兄弟姐妹 & 13 \\
\hline 朋友 & 7 & 陌生人 & 14 \\
\hline
\end{tabular}

2. 你觉得你会信任并借给谁 500 块钱, 如果可能的话? 在以下的备选答案中选 出你的答案, 并在相应的小框内打勾。

\begin{tabular}{|c|c|c|c|}
\hline 熟人/邻居 & 1 & 爷爷姷姷／外公外婆 & 8 \\
\hline 银行经理/会计 & 2 & 丈夫(妻子)/男朋友(文朋友)／情侣 & 9 \\
\hline 最好／最亲密的朋友 & 3 & 父母／监护人 & 10 \\
\hline 老板/领导／老师 & 4 & 心理学家/专业资词师 & 11 \\
\hline 子女㚣／孙子文 & 5 & 亲城 & 12 \\
\hline 同学 /工友／同事 & 6 & 兄弟姐妹 & 13 \\
\hline 朋友 & 7 & 陌生人 & 14 \\
\hline
\end{tabular}


3. 你觉得你会信任并借给谁 10,000 块钱, 如果可能的话? 在以下的备选答 案中选出你的答案, 并在相应的小框内打勾。

\begin{tabular}{|c|c|c|c|}
\hline 熟人ノ邻居 & 1 & 爷爷奶㚴／外公外婆 & 8 \\
\hline 银行经理／会计 & 2 & 才夫(妻子)/男朋友(文朋友)/情侣 & 9 \\
\hline 最好／最亲密的朋友 & 3 & 父母／监护人 & 10 \\
\hline 老板／领导／老师 & 4 & 心理学家/专业咨询师 & 11 \\
\hline 子㚣／孙子交 & 5 & 亲戚 & 12 \\
\hline 同学 /工友/同事 & 6 & 兄弟姐妹 & 13 \\
\hline 朋友 & 7 & 陌生人 & 14 \\
\hline
\end{tabular}

4. 你觉得你会信任并借给谁 100,000 块钱或更多, 如果可能的话? 在以下 的备选答案中选出你的答案, 并在相应的小框内打勾。

\begin{tabular}{|c|c|c|c|}
\hline 熟人/邻居 & 1 & 爷爷好奶／外公外婆 & 8 \\
\hline 银行经理／会计 & 2 & 丈夫(妻子) /男朋友(文朋友)／情侣 & 9 \\
\hline 最好／最亲密的朋友 & 3 & 父母 /监护人 & 10 \\
\hline 老板 /领导／老师 & 4 & 心理学家/专业资词师 & 11 \\
\hline 子女／孙子㚣 & 5 & 亲戚 & 12 \\
\hline 同学/工友/同事 & 6 & 兄弟姐妹 & 13 \\
\hline 朋友 & 7 & 陌生人 & 14 \\
\hline
\end{tabular}


Appendix H (Chinese version): When it Comes to Positive Matters, Whom Would You Trust? 


\section{在涉及积极正面信息的情况下， 你将信任谁?}

说明：这份问卷的目的是为信任研究收集资料。和所有其他的参与者一样，你所提供的信 息完全是不署名的和保密的。对问题的回答也完全没有任何好坏、对错之分。请表达出你 自己的想法和感受，而不是其它。非常感谢你对本研究的支持。

1. 如果你的作业得到了好的评语, 你觉得你会信任并同谁分享这个信息? 在以下 的备选答案中选出你的答案, 并在相应的小框内打勾。

\begin{tabular}{|c|c|c|c|}
\hline 熟人/邻居 & 1 & 爷爷奶奶／外公外婆 & 8 \\
\hline 银行经理/会计 & 2 & 丈夫(妻子)/男朋友(女朋友)/情侣 & 9 \\
\hline 最好／最亲密的期友 & 3 & 父母／监护人 & 10 \\
\hline 老板/领导/老师 & 4 & 心理学家/专业咨词师 & 11 \\
\hline 子文／孙子女 & 5 & 亲墄 & 12 \\
\hline 同学 /工友 /同事 & 6 & 兄弟姐妹 & 13 \\
\hline 朋友 & 7 & 陌生人 & 14 \\
\hline
\end{tabular}

2. 如果你在测验考试中得了一个好分数, 你觉得你会信任并同谁分享这个信息? 在以下的备选答案中选出你的答案, 并在相应的小框内打勾。

\begin{tabular}{|l|l|l|l|}
\hline 熟人/邻居 & 1 & 爷爷奶好/外公外婆 & 8 \\
\hline 银行经理/会计 & 2 & 丈夫(妻子)/男朋友(安朋友)/情侣 & 9 \\
\hline 最好/最亲密的朋友 & 3 & 父母/监护人 & 10 \\
\hline 老板/领导/老师 & 4 & 心理学家/专业咨洵师 & 11 \\
\hline 子女／孙子女 & 5 & 亲戚 & 12 \\
\hline 同学/工友/同事 & 6 & 兄弟姐妹 & 13 \\
\hline 朋友 & 7 & 陌生人 & 14 \\
\hline
\end{tabular}


3. 如果你是你们班上最好/最拔尖的一个学生, 你觉得你会信任并同谁分享这 个信息? 在以下的备选答案中选出你的答案, 并在相应的小框内打勾。

\begin{tabular}{|c|c|c|c|}
\hline 熟人/邻居 & 1 & 爷爷好好／外公外婆 & 8 \\
\hline 银行经理/会计 & 2 & 才夫(妻子)/男朋友(女朋友)／情侣 & 9 \\
\hline 最好／最亲密的朋友 & 3 & 父母／监护人 & 10 \\
\hline 老板/领导／老师 & 4 & 心理学家/专业咨洵师 & 11 \\
\hline 子女／孙子女 & 5 & 亲成 & 12 \\
\hline 同学/工友／同事 & 6 & 兄弟姐妹 & 13 \\
\hline 朋友 & 7 & 陌生人 & 14 \\
\hline
\end{tabular}

4. 如果你是你们学校最好最拔尖的一个学生, 你觉得你会信任并同谁分享这 个信息? 在以下的备选答案中选出你的答案, 并在相应的小框内打勾。

\begin{tabular}{|c|c|c|c|}
\hline 熟人/邻居 & 1 & 爷爷奶好／外公外婆 & 8 \\
\hline 银行经理/会计 & 2 & 才夫(妻子)/男朋友(女朋友)／情侣 & 9 \\
\hline 最好／最亲密的朋友 & 3 & 父母／监护人 & 10 \\
\hline 老板/领导／老师 & 4 & 心理学家/专业咨洵师 & 11 \\
\hline 子女／孙子女 & 5 & 亲墄 & 12 \\
\hline 同学 /工友/同事 & 6 & 克弟姐妹 & 13 \\
\hline 朋友 & 7 & 陌生人 & 14 \\
\hline
\end{tabular}


Appendix I (Chinese version): When it Comes to Negative Matters, Whom Would You Trust? 


\section{在涉及消极/负面信息的情况下， \\ 你将信任谁?}

兑勗：这份问卷的目的是为信任研究收集资料。和所有其他的参与者一样，你所提供的信 息完全是不署名的和保密的。对问题的回答也完全没有任何好坏、对错之分。请表达出你 自己的想法和感受，而不是其它。非常感谢你对本研究的支持。

1. 如果你的作业得到了坏的评语, 你觉得你会信任并同谁分享这个信息? 在以下 的备选答案中选出你的荅案, 并在相应的小框内打勾。

\begin{tabular}{|c|c|c|c|}
\hline 熟人ノ邻居 & 1 & 爷爷奶好／外公外婆 & 8 \\
\hline 银行经理/会计 & 2 & 丈夫(妻子) /男朋友(女朋友)／情侣 & 9 \\
\hline 最好／最亲密的朋友 & 3 & 父母／监护人 & 10 \\
\hline 老板/领导／老师 & 4 & 心理学家/专业资洵师 & 11 \\
\hline 子交／孙子交 & 5 & 亲减 & 12 \\
\hline 同学 /工友／同事 & 6 & 兄弟姐妹 & 13 \\
\hline 朋友 & 7 & 陌生人 & 14 \\
\hline
\end{tabular}

2. 如果你在测验考试中得了一个低分, 你觉得你会信任并同谁分享这个信息? 在 以下的备选答案中选出你的答案, 并在相应的小框内打勾。

\begin{tabular}{|c|c|c|c|}
\hline 熟人／邻居 & 1 & 爷爷奶奶／外公外婆 & 8 \\
\hline 银行经理／会计 & 2 & 丈夫(妻子)／男朋友(文朋友)八情侣 & 9 \\
\hline 最好／最亲密的朋友。 & 3 & 父母／监护人 & 10 \\
\hline 老板/领导／老师 & 4 & 心理学家/专业咨河师 & 11 \\
\hline 子文／孙子㚣 & 5 & 亲戚 & 12 \\
\hline 同学/工友／同事 & 6 & 兄弟姐妹 & 13 \\
\hline 朋友 & 7 & 陌生人 & 14 \\
\hline
\end{tabular}


3. 如果你在专业学习上失败, 你觉得你会信任并同谁分享这个信息? 在以下的备 选答案中选出你的答案, 并在相应的小框内打勾。

的备 远合案中选出你的答案, 并在相应的小栍内打句。

\begin{tabular}{|c|c|c|c|}
\hline 熟人/邻居 & 1 & 爷爷奶奶／外公外婆 & 8 \\
\hline 银行经理/会计 & 2 & 丈夫(妻子)／男朋友(女朋友)／情侣 & 9 \\
\hline 最好／最亲密的朋友 & 3 & 父母／监护人 & 10 \\
\hline 老板 /领导／老师 & 4 & 心理学家/专业咨洵师 & 11 \\
\hline 子㚣/孙子女 & 5 & 亲戚 & 12 \\
\hline 同学 /工友／同事 & 6 & 兄弟姐妹 & 13 \\
\hline 朋友 & 7 & 陌生人 & 14 \\
\hline
\end{tabular}

4. 如果你被学校开除, 你觉得你会信任并同谁分享这个信息? 在以下的备选答案 中选出你的答案, 并在相应的小框内打勾。

\begin{tabular}{|c|c|c|c|}
\hline 熟人/邻居 & 1 & 爷爷奶好／外公外婆 & 8 \\
\hline 银行经理/会计 & 2 & 才夫(妻子) /男朋友（交朋友）／情侣 & 9 \\
\hline 最好／最亲密的朋友 & 3 & 父母／监护人 & 10 \\
\hline 老板/领导/老师 & 4 & 心理学家/专业咨洵师 & 11 \\
\hline 子文／孙子文 & 5 & 亲戚 & 12 \\
\hline 同学/工友/同事 & 6 & 兄弟姐妹 & 13 \\
\hline 朋友 & 7 & 陌生人 & 14 \\
\hline
\end{tabular}


Appendix J (Chinese version): When it Comes to Economic Matters, Who Would Trust You? 


\section{在涉及经济物质的情况下， \\ 谁将信任你?}

说明：这份问卷的目的是为信任研究收集资料。和所有其他的参与者一样，你所提供的信 息完全是不署名的和保密的。对问题的回答也完全没有任何好坏、对错之分。请表达出你 自己的想法和感受，而不是其它。非常感谢你对本研究的支持。

1. 你觉得谁会信任并借给你 10 垬钱, 如果可能的话? 在以下的备选答案中选出 你的答案, 并在相应的小框内打勾。

\begin{tabular}{|c|c|c|c|}
\hline 熟人ノ邻居 & 1 & 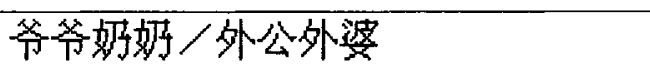 & 8 \\
\hline 银行经理/会计 & 2 & 丈夫(妻子)/男朋友(文朋友)/情侣 & 9 \\
\hline 最好／最亲密的朋友 & 3 & 父母／监护人 & 10 \\
\hline 老板 /领导／老师 & 4 & 心理学家/专业咨洵师 & 11 \\
\hline 子文／孙子文 & 5 & 亲戚 & 12 \\
\hline 同学/工友/同事 & 6 & 兄弟姐妹 & 13 \\
\hline 朋友 & 7 & 陌生人 & 14 \\
\hline
\end{tabular}

2. 你觉得谁会信任并借给你 500 块钱, 如果可能的话? 在以下的备选荅案中选 出你的荅案, 并在相应的小框内打勾。

\begin{tabular}{|c|c|c|c|}
\hline 熟人/邻居 & 1 & 蚉爷好好了/外公外婆 & 8 \\
\hline 银行经理／会计 & 2 & 丈夫(妻子)／要朋友(文朋友)/情侣 & 9 \\
\hline 最好／最亲密的朋友 & 3 & 父母/监护人 & 10 \\
\hline 老板／领导／老师 & 4 & 心理学家/专业咨洵师 & 11 \\
\hline 子女文/孙子交 & 5 & 亲戚 & 12 \\
\hline 同学／工友／同事 & 6 & 兄弟姐妹 & 13 \\
\hline 朋友 & 7 & 陌生人 & 14 \\
\hline
\end{tabular}


3. 你觉得谁会信任并借给你 10,000 块钱, 如果可能的话? 在以下的备选荅 案中选出你的荅案, 并在相应的小框内打勾。

\begin{tabular}{|c|c|c|c|}
\hline 熟人/邻居 & 1 & 爷爷好㚴/外公外婆 & 8 \\
\hline 银行经理 /会计 & 2 & 丈夫(妻子)／男朋友(文朋友)／情侣 & 9 \\
\hline 最好／最亲密的朋友 & 3 & 父母／监护人 & 10 \\
\hline 老板／领导／老师 & 4 & 心理学家/专业咨河师 & 11 \\
\hline 子交／孙子㚣 & 5 & 亲戚 & 12 \\
\hline 同学 /工友/同事 & 6 & 兄弟姐妹 & 13 \\
\hline 朋友 & 7 & 陌生人 & 14 \\
\hline
\end{tabular}

4. 你觉得谁会信任并借给你 100,000 块钱或更多，如果可能的话? 在以 下的备选答案中选出你的答案, 并在相应的小框内打勾。

\begin{tabular}{|c|c|c|c|}
\hline 熟人/邻居 & 1 & 爷斋好姷／外公外婆 & 8 \\
\hline 银行经理／会计 & 2 & 丈夫(妻子)／男朋友(文朋友)／情侣 & 9 \\
\hline 最好／最亲密的朋友 & 3 & 父母／监护人 & 10 \\
\hline 老板/领导／老师 & 4 & 心理学家/专业咨询师 & 11 \\
\hline 子女㚣/孙子女 & 5 & 亲诚 & 12 \\
\hline 同学／工友/同事 & 6 & 兄弟姐妹 & 13 \\
\hline 朋友 & 7 & 陌生人 & 14 \\
\hline
\end{tabular}


Appendix K (Chinese version): When it Comes to Positive Matters,

Who Would Trust You? 


\section{在涉及积极/正面信息的情况下， 谁将信任你?}

说勗：这份问卷的目的是为信任研究收集资料。和所有其他的参与者一样, 你所提供的信 息完全是不署名的和保密的。对问题的回答也完全没有任何好坏、对错之分。请表达出你 自己的想法和感受，而不是其它。非常感谢你对本研究的支持。

1 . 你觉得谁会信任并和你分享这个信息, 如果他／她的作业得到了好的评语? 在以下的备选答案中选出你的答案, 并在相应的小框内打勾。

\begin{tabular}{|c|c|c|c|}
\hline 熟人ノ邻居 & 1 & 爷芧奶好／外公外婆 & 8 \\
\hline 银行经理/会计 & 2 & 丈夫(妻子)／男朋友(文朋友)/情侣 & 9 \\
\hline 最好／最亲密的朋友 & 3 & 父母 /监护人 & 10 \\
\hline 老板／领导／老师 & 4 & 心理学家/专业咨洵师 & 11 \\
\hline 子文／孙子女 & 5 & 亲成 & 12 \\
\hline 同学 /工友 /同事 & 6 & 兄弟姐妹 & 13 \\
\hline 朋友 & 7 & 陌生人 & 14 \\
\hline
\end{tabular}

2. 你觉得谁会信任并和你分享这个信息, 如果他／她在测验考试中得了一个好 分数? 在以下的备选答案中选出你的答案, 并在相应的小框内打勾。

\begin{tabular}{|c|c|c|c|}
\hline 熟人/邻居 & $\overline{1}$ & 爷爷奶好／外公外婆 & 8 \\
\hline 银行经理/会计 & 2 & 丈夫(妻子) /男朋友(文朋友)／情侣 & $\overline{9}$ \\
\hline 最好／最亲密的朋友 & 3 & 父母／监护人 & 10 \\
\hline 老板／领导／老师 & 4 & 心理学家/专业咨洵师 & 11 \\
\hline 子女／孙子文 & 5 & 亲成 & 12 \\
\hline 同学／工友／同事 & 6 & 兄弟姐妹 & 13 \\
\hline 朋友 & 7 & 陌生人 & 14 \\
\hline
\end{tabular}


3. 你觉得谁会信任并和你分享这个信息, 如果他／她是他（她）们班上最好/最 拨尖的一个学生? 在以下的备选答案中选出你的答案, 并在相应的小框内打勾。

\begin{tabular}{|c|c|c|c|}
\hline 熟人/邻居 & 1 & 爹爷奶好／外公外婆 & 8 \\
\hline 银行经理／会计 & 2 & 丈夫(妻子)／男朋友(交朋友)／情侣 & 9 \\
\hline 最好／最亲密的朋友 & 3 & 父母/监护人 & 10 \\
\hline 老板 /领导/老师 & 4 & 心理学家/专业咨词师 & 11 \\
\hline 子交/孙子㚣 & 5 & 亲戚 & 12 \\
\hline 同学 /工友/同事 & 6 & 兄弟姐妹 & 13 \\
\hline 朋友 & 7 & 陌生人 & 14 \\
\hline
\end{tabular}

4. 你觉得谁会信任并和你分享这个信息, 如果他／她是他（她）们学校最好/最 拔尖的一个学生? 在以下的备选答案中选出你的答案, 并在相应的小框内打勾。

\begin{tabular}{|c|c|c|c|}
\hline 熟人/邻居 & 1 & 芧爷好姷／外公外婆 & 8 \\
\hline 银行经理／会计 & 2 & 丈夫(妻子)/男朋友(交朋友)/情侣 & 9 \\
\hline 最好／最亲密的朋友 & 3 & 父母／监护人 & 10 \\
\hline 老板 /领导／老师 & 4 & 心理学家/专业咨词师 & 11 \\
\hline 子女／孙子女 & 5 & 亲戚 & 12 \\
\hline 同学／工友／同事 & 6 & 兄弟姐妹 & 13 \\
\hline 朋友 & 7 & 陌生人 & 14 \\
\hline
\end{tabular}


Appendix L (Chinese version): When it Comes to Negative Matters, Who Would Trust You? 


\section{在涉及消极/负面信息的情况下， \\ 谁将信任你?}

兑明：这份问卷的目的是为信任研究收集资料。和所有其他的参与者一样，你所提供的信 息完全是不署名的和保密的。对问题的回答也完全没有任何好坏、对错之分。请表达出你 自己的想法和感受，而不是其它。非常感谢你对本研究的支持。

1. 你觉得谁会信任并和你分享这个信息, 如果他／她的作业得到了坏的评语? 在 以下的备选荅案中选出你的答案, 并在相应的小框内打勾。

\begin{tabular}{|c|c|c|c|}
\hline 熟人/邻居 & 1 & 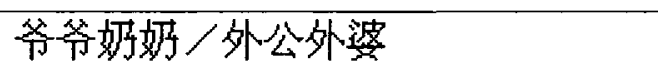 & 8 \\
\hline 银行经理／会计 & 2 & 丈夫(妻子)／男朋友(文朋友)／情侣 & 9 \\
\hline 最好／最亲密的朋友 & 3 & 父母／监护人 & 10 \\
\hline 老板／领导／老师 & 4 & 心理学家/专业咨洵师 & 11 \\
\hline 子文／孙子文 & 5 & 亲成 & 12 \\
\hline 同学／工友／同事 & 6 & 兄弟姐妹 & 13 \\
\hline 朋友 & 7 & 陌生人 & 14 \\
\hline
\end{tabular}

2.你觉得谁会信任并和你分享这个信息, 如果他／她在测验考试中得了一个低 分? 在以下的备选答案中选出你的答案, 并在相应的小框内打勾。 2.你觉得谁会信任并和你分享这个信息, 如果他/她在测验考试中得了一个低 分? 在以下的备选答案中选出你的荅案, 并在相应的小框内打勾。

\begin{tabular}{|c|c|c|c|}
\hline 熟人/邻居 & 1 & 爷爷好好子/外公外婆 & 8 \\
\hline 银行经理/会计 & 2 & 丈夫(妻子)／男朋友(文朋友)/情侣 & 9 \\
\hline 最好／最亲密的朋友 & 3 & 父母/监护人 & 10 \\
\hline 老板／领导／老师 & 4 & 心理学家/专业咨洵师 & 11 \\
\hline 子立 /孙子交 & 5 & 亲或 & 12 \\
\hline 同学 $/ 工$ 友 $/$ 同事 & 6 & 兄弟姐妹 & 13 \\
\hline 朋友 & 7 & 陌生人 & 14 \\
\hline
\end{tabular}


3. 你觉得谁会信任并和你分享这个信息, 如果他／她在专业学习上失败? 在以 下的备选答案中选出你的答案, 并在相应的小框内打勾。

\begin{tabular}{|l|l|l|l|}
\hline 熟人/邻居 & 1 & 爷爷好好/外公外婆 & 8 \\
\hline 银行经理/会计 & 2 & 丈夫(妻子)/男朋友(女朋友)/情侣 & 9 \\
\hline 最好/最密的朋友 & 3 & 父母/监护人 & 10 \\
\hline 老板/领导/老师 & 4 & 心理学家/专业咨洵师 & 11 \\
\hline 子女／孙子女 & 5 & 亲成 & 12 \\
\hline 同学/工友/同事 & 6 & 兄弟姐妹 & 13 \\
\hline 朋友 & 7 & 陌生人 & 14 \\
\hline
\end{tabular}

4 . 你觉得谁会信任并和你分享这个信息, 如果他 /她被学校开除? 在以下的备选 荅案中选出你的答案, 并在相应的小框内打勾。

\begin{tabular}{|c|c|c|c|}
\hline 熟人/邻居 & 1 & 爷爷奶好／外公外婆 & 8 \\
\hline 银行经理/会计 & 2 & 才夫(妻子)/男朋友(女朋友)／情侣 & 9 \\
\hline 最好／最亲密的朋友 & 3 & 父母／监护人 & 10 \\
\hline 老板/领导／老师 & 4 & 心理学家/专业咨洵师 & 11 \\
\hline 子女／孙子女 & 5 & 亲戚 & 12 \\
\hline 同学/工友/同事 & 6 & 兄弟姐妹 & 13 \\
\hline 朋友 & 7 & 陌生人 & 14 \\
\hline
\end{tabular}

